\title{
Ultra Low Power Wake-Up Radios: A Hardware and Networking Survey
}

\author{
Rajeev Piyare, Member, IEEE, Amy L. Murphy, Senior Member, IEEE, Csaba Kiraly, Member, IEEE, \\ Pietro Tosato, Student Member, IEEE, and Davide Brunelli, Senior Member, IEEE
}

\begin{abstract}
1 Abstract-In wireless environments, transmission and 2 reception costs dominate system power consumption, motivating 3 research effort on new technologies capable of reducing the 4 footprint of the radio, paving the way for the Internet of 5 Things. The most important challenge is to reduce power 6 consumption when receivers are idle, the so called idle-listening 7 cost. One approach proposes switching off the main receiver, 8 then introduces new wake-up circuitry capable of detecting 9 an incoming transmission, optionally discriminating the packet 10 destination using addressing, then switching on the main radio 11 only when required. This wake-up receiver technology represents 12 the ultimate frontier in low power radio communication. In 13 this paper, we present a comprehensive literature review of 14 the research progress in wake-up radio (WuR) hardware and 15 relevant networking software. First, we present an overview of 16 the WuR system architecture, including challenges to hardware 17 design and a comparison of solutions presented throughout the 18 last decade. Next, we present various medium access control and 19 routing protocols as well as diverse ways to exploit WuRs, both 20 as an extension of pre-existing systems and as a new concept to 21 manage low-power networking.
\end{abstract}

Index Terms-Wake-up radio, MAC protocols, energy effi23 ciency, multichannel, asynchronous communication, Internet of 24 Things, survey, green networking.

\section{INTRODUCTION}

26 THE INTERNET of Things (IoT) offers a new Internet ${ }_{27}$ frontier considering networks between smart physical 28 objects or "Things", which are embedded with sensors, actu29 ators, and/or processing capabilities [1]. IoT provides novel so applications for various fields such as Smart Cities, build31 ing automation, domotics, logistics, Smart Grid, e-Health, and 32 agriculture [2].

33 A founding pillar of the IoT concept is the availability 34 of low-cost devices with low-power wireless communication 35 capabilities, often deployed as part of a larger Wireless Sensor ${ }_{36}$ Network (WSN) [3], to provide both sensing and actuation 37 capabilities. These devices are usually powered by batteries 38 with restricted size and capacity [4], and thus have limited lifeз9 time requiring careful power management. With the increase

Manuscript received August 11, 2016; revised December 29, 2016 and April 26, 2017; accepted June 15, 2017. (Corresponding author: Amy L. Murphy.)

R. Piyare, A. L. Murphy, and C. Kiraly are with Fondazione Bruno Kessler, 38123 Trento, Italy (e-mail: piyare@fbk.eu; murphy@fbk.eu; kiraly@fbk.eu).

P. Tosato and D. Brunelli are with the University of Trento, 38123 Trento, Italy (e-mail: pietro.tosato@unitn.it; davide.brunelli@unitn.it).

Digital Object Identifier 10.1109/COMST.2017.2728092 in the number of IoT devices, replacing or recharging batteries frequently will not only be costly but infeasible as well. Therefore, prolonging the lifetime of these devices, or even better achieving perpetual operation, becomes fundamental for the realization of the IoT vision.

Traditionally, these problems have been addressed by the 45 introduction of low-power radios and of duty-cycling Medium 46 Access Control (MAC) protocols [5]. Notwithstanding, one ${ }_{47}$ of the most power hungry tasks performed by these nodes is 48 low-power wireless communication. In most applications, its 49 consumption far exceeds that of sensing, actuation, and pro- 50 cessing, and became the main bottleneck in extending device lifetime.

Recent developments in CMOS power consumption have led to the birth of a new design paradigm of wake-up radios (WuRs) to further reduce power consumption and, in combination with energy harvesting [6], [7], reach the goal of the perpetual operation.

\section{A. From Duty-Cycling MACs to Wake-Up Radios}

The main reason duty-cycling MACs alone can not sufficiently extend the lifetime of a node is that the consumption of low-power wireless radios is almost the same when listening for transmissions as while transmitting. For example, the widely used CC2420 radio module consumes $21.8 \mathrm{~mA}$ in listening mode and $19.5 \mathrm{~mA}$ in data transmission mode [8]. If such a radio would be always-on (listening for other trans- 65 missions or transmitting) it would deplete reasonable sized 66 batteries in less than a week.

During duty-cycling, the nodes are periodically put into 68 sleep mode and are woken up only to transmit or to receive. 69 Unfortunately, the so called duty-cycling ratio (the ratio of 70 time the radio is in transmit or receive mode to time off) 71 cannot go arbitrarily low, due to:

(i) idle listening: occurs when the node monitors the communication medium for ongoing transmissions, but there is no data to be received by the node. Since nodes must listen periodically to limit data latency, there is a listening power consumption that cannot be avoided, even in low data traffic scenarios.

(ii) overhearing: occurs when a node receives packets from 79 its neighbors that are not intended for that node, leading 80 to energy waste, especially when the network density is 81 high and the data traffic is heavy.

48

49




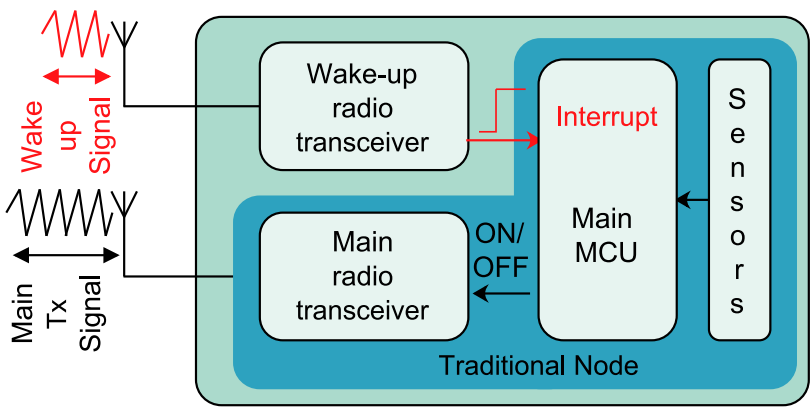

Complete Node with Wake-up Radio

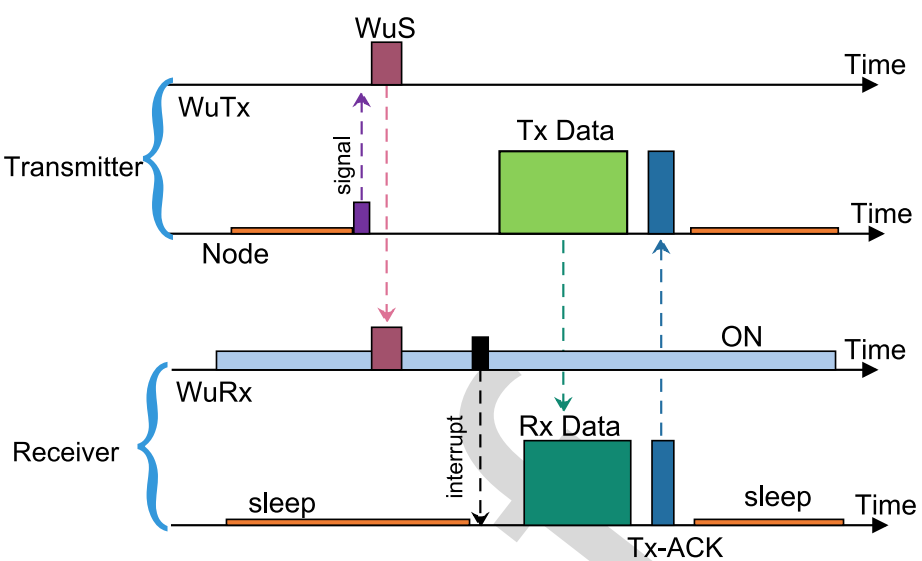

(b)

Fig. 1. (a) Overall Wake-up Radio architecture. The blue region indicates the traditional node integrated with the Wake-up Radio. (b) Remote triggering using wake-up radio scheme.

83 Due to the sleep intervals, duty-cycling protocols also intro84 duce significant data latency since no information can be sent 85 or received until the nodes wake-up.

86 Finally, duty-cycling MAC protocols must either maintain 87 time synchrony to make sure transmitters send when receivers 88 are awake, which induces a time synchronization overhead, 89 or in the case of asynchronous operation the MAC protocol 90 must employ continuous (or multiple) transmissions to ensure 91 reception. The longer the sleep interval of the receiver, the 92 longer the continuous transmission must be, dictating a lower${ }_{93}$ bound on achievable duty-cycles.

94 These design compromises have led the sensor network 95 community to design and implement various MAC protocols 96 resulting in a "MAC Alphabet Soup" for sensor networks [9] 97 each targeting different scenarios and offering different com98 promises throughout the design space of energy consumption, 99 latency, throughput, and fairness. Nevertheless, duty cycling 100 protocols may not be suitable for delay sensitive and event${ }_{101}$ driven applications, and prolonging device lifetime requires 102 extreme compromises in other dimensions of the design space, 103 limiting the applicability of the technique.

104 The introduction of wake-up radios aims to provide a novel 105 hardware solution with listening power consumption orders 106 of magnitude lower than that of low-power radios, promising 107 results towards eliminating the aforementioned problems of 108 idle listening, overhearing, continuous transmissions, and data 109 latency.

110 In a WuR architecture, as shown in Fig. 1 (a), an ultra-low 111 power, secondary radio module with a receiver consuming $a$ 112 few micro watts of power is along side the primary, low-power 113 radio. Since its power consumption is several orders of mag114 nitude lower than that of a traditional low-power radio, the $115 \mathrm{WuR}$ can be kept always-on, leading to a use in contrast to 116 the duty cycling operation descried earlier for the main radio. ${ }_{117}$ One modality in which the WuR can be used is illustrated in ${ }_{118}$ Fig. 1 (b). In this setting, the main radio is kept in a deep 119 sleep, or off mode, until it is needed. Instead when a node has 120 a data packet to send, it sends a special packet known as a 121 wake-up signal (WuS) using its wake-up transmitter (WuTx). 122 The always-on wake-up receiver (WuRx) detects this $\mathrm{WuS}$, and generates an interrupt to the main node's micro-controller ${ }_{123}$ to switch it from sleep to an active mode. Subsequently, the ${ }_{124}$ main micro-controller turns on the main radio transceiver to 125 exchange data packets with the other node in a conventional 126 manner.

This seemingly simple and obvious mode of operation has 128 been made possible by recent advances in CMOS power con- 129 sumption, allowing both the implementation of the ultra low 130 power analog front-end to receive the $\mathrm{WuS}$ as well as a low ${ }_{131}$ power digital component used inside the WuR for address ${ }_{132}$ decoding.

\section{B. Wake-Up Radio: Benefits and Design Trade-Offs}

As mentioned previously, idle listening is a significant con- ${ }_{135}$ tributor to the overall energy consumption of duty cycling 136 nodes. With the introduction of a WuRx with orders of mag- 137 nitude lower consumption, the WuR approach minimizes this ${ }_{138}$ unnecessary energy wastage, as the main radio and the node ${ }_{139}$ will be activated only when there is an actual transmission. $\quad 140$

In addition, some WuRs add circuitry for an addressing 141 mechanism that can be used to solve the issue of overhear- 142 ing by decoding an address embedded in the packet, waking ${ }_{143}$ up only a specific node rather than the entire neighborhood. ${ }_{144}$

Further, since the WuRx can be always-on, the node can 145 operate in a purely asynchronous manner, activating the main 146 radio on-demand, without requiring continuous transmissions. ${ }_{147}$

Finally, since the time taken to trigger the main node is on 148 the order of milliseconds (ms), the latency problem faced by 149 duty-cycling MAC protocols is also reduced. 150

While the concept the WuR seem simple and the benefits 151 look promising, the hardware implementation and its usage as ${ }_{152}$ part of the larger system present several challenges and design ${ }_{153}$ trade-offs.

At the hardware design level, achieving listening with very 155 low power consumption places limits on RX processing and 156 on the components that can be used in the WuRx. Various 157 hardware options had been explored in literature exploring 158 a wide range of options, including some that are not radio 159 frequency (RF) based, e.g., optical or acoustic. 
161 Strict bounds on power consumption also limit the choice 162 of modulation schemes and receiver complexity, which, as 163 a consequence, limit receiver sensitivity, and ultimately the 164 achievable communication range. As the main radio is trig165 gered by the WuR, this range limitation of the WuR inherently 166 limits the communication, regardless of the main radio's capa167 bilities. As we will show throughout our survey, various 168 compromises have been taken in this regard, from focus169 ing on short-range scenarios (Body Area Networks), to using 170 out-of-band sub-GHz WuS, to using greatly increased WuTx 171 power.

172 As far as the MAC protocol is concerned, pure asyn173 chronous operation enabled by the always-on WuRx largely 174 simplifies protocol design. However, the development of new $175 \mathrm{WuR}$ specific MAC protocols are required, taking into account 176 the dual radio setup of the WuR architecture.

\section{C. Contribution and Related Work}

178 This paper offers:

179 (i) An extensive survey and classification of the state of 180 the art in wake-up receiver prototypes implemented and 181 tested since 2002, specifically 75 RF based wake-up 182 radios (Table VIII) and 10 non-RF based prototypes 183 (Table IX) 192 characteristics of the wake-up technology such as power con193 sumption, sensitivity and data rates, but focusing only on 194 characteristics at the hardware layer. While we also present the 195 hardware, we offer insight into its impact on the networking 196 stack software.

197 Alternate work considers the validity of the combination of 198 wake-up radios and energy harvesting [12]-[14].

199 Similarly, several studies have emphasized the 200 benefits of wake-up radios for extending node life201 time [11], [15], [16], while also improving reliability 202 and reducing latency [17], [18]. Our work compliments and 203 extends these by reporting on several wake-up radios that 204 directly harvest energy from the wake-up signal, referred to 205 as passive in Table VIII, as well as on the consumption values 206 of the receivers, a critical element for considering them as a 207 component in a system with energy-harvesting.

208 On the software side, the last decade has seen a plethora of 209 low-power communication protocols [19], especially focused 210 on the MAC layer [5], [20]-[22] or on general energy conser211 vation schemes [23]. A brief survey of wake-up receivers for 212 WSNs offered an introduction to the technology [24], focusing 213 on software only at the MAC layer. Routing has been studied 214 in general for WSNs in isolation [25]-[28], or in cross-layer 215 solutions [29], [30]. Nevertheless these surveys do not focus
TABLE I

ACRONYMS FOR WAKE-UP RADIO TECHNOLOGY

\begin{tabular}{|l|l|}
\hline WuR & $\begin{array}{l}\text { wake-up radio, the secondary low- } \\
\text { power module }\end{array}$ \\
\hline WuRx & wake-up receiver \\
\hline WuTx & wake-up transmitter \\
\hline WuS & $\begin{array}{l}\text { wake up signal, the message sent } \\
\text { by the WuTx }\end{array}$ \\
\hline
\end{tabular}

on the unique properties of the wake-up radio technology, and 216 the impact it has on this layer.

\section{Structure of This Article}

The remainder of this paper is organized as follows: 219 Section II depicts the main characteristics of a wake-up radio. 220 Section III discusses the design space and architecture of 221 wake-up radios followed by some of the main implementation 222 requirements when designing wake-up radio based systems. 223 Sections IV and V discuss the state-of-the-art wake-up radio 224 hardware designs and comparative analysis between each char- 225 acteristic, respectively. The integration of different medium 226 access control and routing protocols that are based on wake- 227 up radios are presented in Sections VI and VII. In Section VIII 228 we briefly discuss some of the application scenarios that can 229 benefit from wake-up radios. Finally, in Section X we conclude 230 this survey with open research issues.

\section{WAKe-Up RADIO Defining CHARACTERISTICS AND REQUIREMENTS}

Before we begin, we summarize in Table I the key termi- 234 nology we use throughout our survey to identify components 235 of the wake-up technology.

236

The technology and design considerations for the WuR play ${ }_{237}$ a key role in determining the efficiency of low power sensor 238 networks. For the WuR to operate effectively as part of the 239 larger system in a multi-user environment, it should consider 240 the following design points:

241

(i) Power consumption: The most important feature of the 242 WuR is its low power consumption in active mode. In 243 fact, as its use requires the addition of new hardware on 244 top of the main node, the device itself must consume 245 no more than tens of micro-watts. Specifically WuR's 246 active power should be below that of the main radio's 247 sleep power [31] to provide a positive balance between 248 power saved and used. This is the main specification 249 driving WuR design.

(ii) Time to wake-up: The node attached to the WuR must ${ }_{251}$ wake-up with minimum latency upon reception of $\mathrm{WuS} 252$ to avoid latency incurred from multi-hops toward the 253 sink and to increase the overall responsiveness of a 254 purely asynchronous network. A range of protocols and 255 applications can benefit from WuR based systems pro- 256 vided that the latency is low. For example, applications ${ }_{257}$ in health-care have strict latency requirements and can- 258 not support introducing long delays due to the wake up 259 procedure.

260

(iii) False wake-ups and interference: If all nodes in a sen- 261 sor network rely on the same wake-up strategy, when 262 
the WuTx tries to wake-up a node, it will trigger all the nodes in the neighborhood causing significant energy waste. This causes unnecessary activation of many nodes that should be avoided. There are two possible sources of false wake-ups: 1) nodes waking up when receiving a $\mathrm{WuS}$ intended for another node, and 2) interference from nearby devices operating at the same frequency. To tackle the first, the WuR can employ a node addressing and decoding capability to trigger only the intended node. This allows the WuRx to avoid generating an interrupt if the WuS was not intended for it, however it introduces complexity and often consumption at the WuRx. Second, interference and background noise that can result in erroneous wake-ups must be filtered. A WuRx must have enough local processing capability to differentiate a WuS from ambient interference, without using the main node's processor. Due to the low power budget, only basic modulation techniques can be used requiring a simple receiver structure [32]. Modulation schemes such as on-off keying (OOK), pulse width modulation (PWM) or amplitude shift keying (ASK) can be used to reduce the possibility of devices interfering with each other. A preamble can be used to differentiate noise from a valid $\mathrm{WuS}$, thus avoiding false wake-ups. In addition, the WuS must not be missed by the targeted node, as retransmissions are costly in terms of power consumption and latency. To ensure this, a feedback loop such as WuS acknowledgment (WuS-ACK) can be employed by the WuRxs indicating the successful reception of the WuS.

(iv) Sensitivity and range: In WuR design, receiver sensitivity is an important parameter as it provides the lowest power level at which the receiver can detect a WuS. Generally, high sensitivity requires more power hungry electronics at the receiver side, thus high power demand. In contrast, low sensitivity for the same communication range will require high radiated power at the transmitter side. Because of this, sensitivity requirements often leads to over-design to ensure reliable communication in adverse conditions. When the WuR is used to trigger a higher power radio, ideally it should have the same range. Unfortunately this is not reasonable with the power constraints, therefore, most WuR designs target tens of meters of communication range to support many application scenarios [33]. Very short communication ranges make WuR impractical as high node densities would be required to cover a short distance in a multi-hop fashion increasing node and energy costs. Another side effect of a short communication range is the increase in the hop count messages must traverse to reach the sink, increasing the overall data latency. The wake-up range that can be achieved with most current WuR designs is typically around $30 \mathrm{~m}$ a value that can be improved by using techniques such as antenna diversity [34] and directional antennas [35].

(v) Data rate: The overall power expenditure of a node is not only a function of physical layer properties such as carrier frequency, radio architecture, and the choice of the antenna, but is also a function of the amount of time 321 the radio spends to deliver the data packet over the air. 322 This time depends on the data rate supported by the ${ }_{323}$ WuTx and the protocol overhead to establish and main- 324 tain the communication link. Data rate is, therefore, one 325 of the key factors defining the power consumption of 326 WuRs. For example, a WuR with $100 \mathrm{kbps}$ will con- 327 sume almost half the power of a $50 \mathrm{kbps}$ WuR for the ${ }_{328}$ same payload size. For a WuTx with low data rate, the 329 bit duration and the power required to send the $\mathrm{WuS} 330$ will be significantly higher. Due to the longer bit dura- 331 tion, the modulation will keep the transmitter active for a 332 longer time. On the WuRx side, the time and the energy $з з 3$ required to generate the wake-up interrupt will also be 334 significantly higher as the receiver and the demodula- 335 tion circuitry will be active until the transmission ends. 336 A higher data rate can be seen as a way to improve ${ }_{337}$ energy efficiency and to achieve faster wake-up. While 338 a high data rate reduces wake-up latency, a longer bit $3 з 9$ duration increases the communication range and the reli- 340 ability of the WuS. At a lower data rate the energy per 341 bit exhibited by the transmitter is higher, which can be 342 accumulated by the WuRx while receiving the WuS. A ${ }_{343}$ high data rate is not strictly required by the WuR, espe- 344 cially if it is only used as a triggering device as only a 345 few bytes of data are required.

(vi) Cost and size: To integrate the WuR into existing sensor ${ }^{347}$ nodes, it should be cost effective. To make the WuR ${ }_{348}$ feasible [24], the cost of this additional hardware should 349 be in the range of $5-10 \%$ of the cost of the complete 350 sensor node. This is, nevertheless, a loos requirement, 351 as some applications can support higher costs if gains 352 are sufficient. Further, standard off-the-shelf components 353 can be used to speed the development and to reduce 354 the overall cost as compared to designing a single chip 355 solution.

(vii) Frequency regulation: Finally, WuR designs should 357 adhere to frequency regulations in industrial, scientific 358 and medical (ISM) bands. It must also comply with 359 communication standards such as the maximum allowed 360 effective radiated power (ERP) used to transmit WuS. 361

\section{ARChitecture AND TAXONOMY OF WuRs 362}

We begin this section by presenting a generic architecture 363 for WuRs and the building blocks that makeup the complete 364 hardware solution. We discuss the functionality of different 365 hardware components and how these devices can be powered ${ }_{366}$ and interfaced with traditional sensor nodes. We then move on 367 to present a taxonomy of WuRs, illustrated in Fig. 4, show- 368 ing multiple dimensions that distinguish the designs from one 369 another.

\section{A. Generic Architecture of WuRs}

While WuRs can be constructed in many different ways, 372 each exposing different performance and peculiarities, there ${ }_{373}$ are some common building blocks utilized by all designs. Two 374 distinguished implementation approaches have been identified, 375 


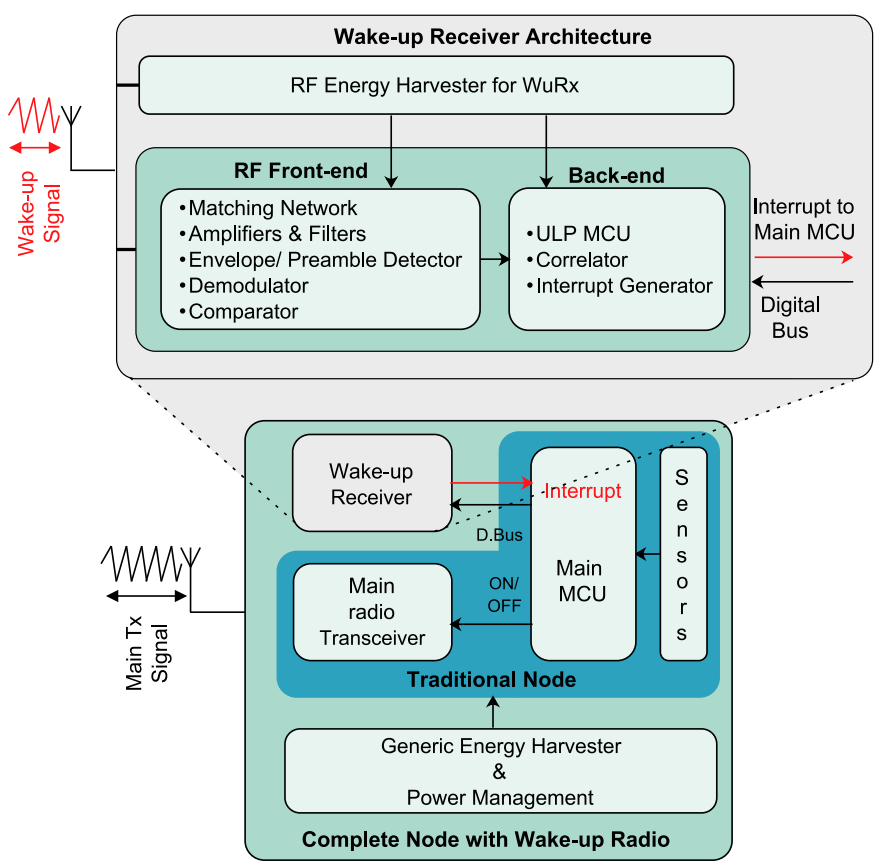

Fig. 2. Expanded view of the generic wake-up receiver architecture with energy harvesting capabilities.

376 i.e., prototypes constructed using off-the-shelf discrete compo377 nents and implementations that exploit CMOS technology for 378 constructing integrated circuits. Power consumption is one of 379 the driving factors behind the use of WuRs due to the energy 380 saving that it can provide. Typically, CMOS implementations 381 achieve improved performance because they better integrate all 382 the components directly on silicon, i.e., more dense integrated 383 circuits result in smaller IC footprints for the same function, 384 hence consuming less power. On the other hand, when using 385 discrete components there are more constraints on each sin386 gle component selected to build the circuit resulting in worse 387 average performance than CMOS-based designs.

388 Fig. 2 illustrates the current architecture and the different 389 functional blocks that form a complete WuRx. This architec390 ture is divided into two sections: the $R F$ front-end and the 391 back-end.

392 The WuS is first received by the RF front-end via the 393 antenna and then passes through the matching network that 394 filters and boosts the incoming WuS. After input matching, 395 an envelope detector performs signal detection and conver396 sion to baseband signal making the circuit simpler and energy 397 efficient. Then, the signal passes through the amplifiers, often 398 the low noise amplifier (LNA) for increasing the sensitivity of 399 the receiver by amplifying weak signals while meeting noise 400 requirements. The LNA dominates in terms of power con401 sumption. Therefore, while designing ultra-low-power WuRxs 402 it is essential to eliminate some, if not all, of these power403 hungry RF components, to reduce power consumption. The 404 voltage multiplier rectifies the RF energy and converts this 405 input signal into a direct current (DC) signal. Usually, the 406 voltage multiplier is constructed by cascading capacitors and 407 zero-bias Schottky diodes. The more energy in the RF signal, 408 the greater the voltage change at the output of the rectifier,

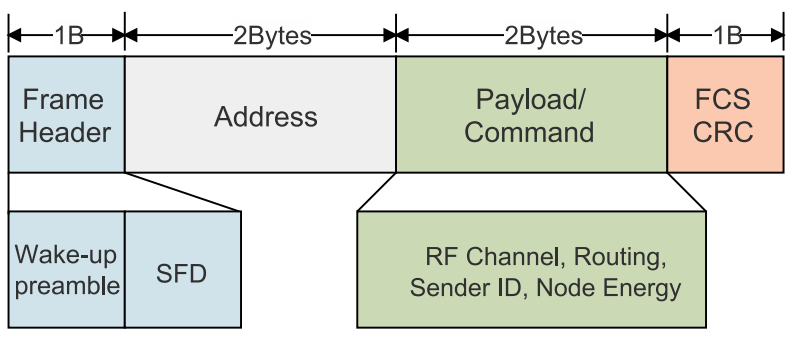

Fig. 3. Typical wake-up signal packet structure.

which is sensed using a comparator. When there is enough 409 energy to trigger the comparator, the back-end is able to issue 410 an interrupt to the main micro-controller. This back-end can ${ }_{411}$ also consist of an ultra-low power micro-controller or cor- ${ }_{412}$ relator circuit that decodes and filters the node address and ${ }_{413}$ generates an interrupt.

414

From the energy point-of-view, one of the hurdles is to 415 supply sufficient energy to operate these devices in a self- 416 sufficient manner without replacing batteries frequently. One 417 of the approaches to achieve this is through Wireless Energy 418 Harvesting (WEH). As illustrated in Fig. 2 the subsystem can 419 include one or more energy harvesters that convert the ambient 420 energy into electrical energy. The Generic Energy Harvester ${ }_{421}$ module that can power the complete node (including the ${ }_{422}$ WuRx, the main transceiver, the main MCU and the sensors) ${ }_{423}$ exploiting different energy sources such as magnetic, solar, ${ }_{424}$ wind, and mechanical vibrations. Also a separate and stan- ${ }_{425}$ dalone RF Energy Harvester, dedicated only for the WuRx, 426 can be employed making the subsystem fully passive, i.e., the ${ }_{427}$ energy can be scavenged from the incoming WuS itself. The ${ }_{428}$ RF-EH unit consists of an antenna and a power management ${ }_{429}$ unit (PMU). The PMU basically controls the power supplied to 430 other blocks of the WuRx. In some applications it is possible ${ }_{431}$ to directly power the WuRx using the harvested energy from ${ }_{432}$ the $\mathrm{WuS}$ without energy storage, however, this may not be a ${ }_{433}$ viable solution. An alternative would be to include a storage ${ }_{434}$ component such as rechargeable batteries or super-capacitors 435 acting as an energy buffer for the subsystem. The main purpose ${ }_{436}$ of this storage component will be to accumulate and preserve ${ }_{437}$ the harvested energy for later use, thus supporting variations in 438 the RF power level emitted by the WuTx. The wake-up range 439 is relatively short due to free space path loss, low sensitivity, 440 and efficiency of power harvesting at the WuRx. As a result, ${ }_{441}$ the $\mathrm{WuS}$ is usually transmitted at high power. ${ }_{442}$

The wake-up transmitter, which is usually not detailed in ${ }_{443}$ the literature, also plays an important role from the system 444 point of view. Most of the works mentioned in this survey use 445 the standard node's transmitter as a WuTx such as CC2420 or 446 CC1101 [11], [15], [36]-[40].

447

Finally, we briefly address the content of the WuS, 448 whose packet structure must meet compliance requirements 449 and standards to be used by different technologies. Recent 450 attempts [41] have been made to standardize this for WuRs in ${ }_{451}$ medical applications.

A typical WuS packet is illustrated in Fig. 3: 453

(i) Frame Header: The frame header consists of the wake- ${ }_{454}$ up preamble and start frame delimiter (SFD), a standard 455 


\section{B. Taxonomy Overview}

For the purposes of this survey, we identify four major 480 dimensions for classifying a WuR: power source, addressing 481 capability, channel usage and communication medium. Fig. 4 482 shows multiple options for each of these dimensions and maps, 483 when possible, the WuRs from Tables VIII and IX. We address 484 each major dimension, beginning with power, as it has the 485 most significant impact on system efficiency.

byte pattern agreed between the transmitter and the the frame and when to start decoding the contents of the packet. The size of the SFD is typically fixed at $1 \mathrm{~B}$.

tination node ID for identifying the intended receiver. While most designs in our literature survey use node IDs up to 2 bytes [38], [42], the size of this field can be varied depending on the capabilities of the WuRx as discussed below. One of the dimensions of our taxonomy, described next, considers the benefits and costs of addressing inside the packet.

cation data, command or extra instructions specified by the user or application.

frame check sequence (FCS) using a cyclic redundancy code (CRC) is applied. While simple, the CRC provides a high degree of error detection at high speed.

(i) Power (Passive): While the WuR requires power to receive a signal, it does not require continuous power. Instead, it can harvest energy, e.g., from the ambient environment or from the incoming wake-up signal itself (Fig. 2). The latter case places a burden on the transmitter side as the WuTx must modulate and transmit the WuS long enough, typically a few seconds, for the WuRx to detect the signal and accumulate enough energy to power the trigger circuitry. The longer the WuTx is active, the more power is consumed. Moreover, this process requires additional hardware at the WuRx side, thus increasing circuit complexity. The process of accumulating energy also delays the wake-up of the main node, affecting network performance by increasing latency and reducing data throughput. Although passive WuRs are energy efficient and offer extended lifetimes, they often have a shorter operating range than active WuRs, typically only a few meters.

(ii) Power (Active): To address the constraints of passive $\mathrm{WuR}$, the majority of research efforts focus on fullyactive WuRs that receive a continuous, external power supply either using batteries or a renewable energy harvester hosted on the main node. The objective of this design is to increase sensitivity, providing longer operational ranges with very low power consumption. $65 \%$ of the prototypes that we present in this survey are 511 active WuRs.

(iii) Power (Semi-active): In semi-active WuRs, a minority 513 of the components of the receiver, e.g., correlator, com- 514 parator and decoder, require continuous power from an 515 external source while the RF front-end remains passive. 516 Next we consider the recipient of the WuS, specifically 517 whether it can be broadcast-only, with the intent to reach all 518 nodes in range, or can contain an address as shown in Fig. 3, 519 intended for a node with a specific ID. 520

(i) Addressing (ID-Based): Optionally, the $\mathrm{WuS}$ can contain 521 a bit sequence, typically 8 to 16 bits, for selective node 522 addressing. This increases the size of the packet, but 523 reduces false wake-up and thus overall system energy 524 consumption. After reception of the WuS, the WuRx ${ }_{525}$ checks if the signal is intended for it. If so, it triggers and 526 wakes up the main node for data reception. This scheme 527 is referred to as ID-based wake-up and is mostly used to 528 construct unicast-based systems. It should be noted that ${ }_{529}$ energy is consumed to decode a wake-up packet and this 530 is typically performed by an external, low-power micro- 531 controller. Further, the length of the address encoding 532 affects performance. While a long address code is more ${ }_{533}$ robust against false wake-ups, it requires a long trans- 534 mit time, hence more power is consumed. Studies [43] 535 consider the trade-off between the length of the wake-up 536 signal and the energy savings, revealing that the energy ${ }_{537}$ used to send the selective wake-up signal only pays off if 538 many nodes are not falsely woken up. In other words, the 539 energy required to transmit the wake-up signal is higher 540 than the energy lost during false-wake up. For low den- 541 sity networks where little data is exchanged, the extra ${ }_{542}$ cost of ID-based addressing may not be worthwhile. $\quad{ }_{543}$

(ii) Addressing (Broadcast): When the entire neighborhood 544 of nodes receives the wake-up signal, the scheme is 545 referred to as broadcast based wake-up. Broadcast based 546 wake-up can reduce the data latency w.r.t. ID-based 547 systems since the receiving node need not decode a 548 wake-up packet to analyze the recipient ID, but can 549 instead immediately trigger its main radio transceiver 550 after receiving the preamble. However, this is potentially 551 expensive in terms of total system power consumption 552 as all neighboring nodes are woken up.

\section{3}

Next, we turn to how the WuR transceiver utilizes the chan- 554 nel for WuS transmission. Note that the choice of channel or 555 frequency depends on the application and the device to which 556 the WuR is attached.

(i) Channel (In-Band): In in-band communication, the main 558 node's transceiver and the WuR use the same frequency 559 band, i.e., either $2.4 \mathrm{GHz}$ or sub-GHz and can share the 560 same antenna. This technique is cheaper as there is no 561 need for a separate antenna.

562

(ii) Channel (Out-of-Band): In out-of-band systems, the ${ }_{563}$ main node and the WuRx are equipped with sepa- 564 rate transceivers, each operating at different frequencies. 565 For instance, the WuR prototype presented in [38] 566 operates at $868 \mathrm{MHz}$ while the main data radio 567 operates at $2.4 \mathrm{GHz}$ band. Using frequency or code 568 


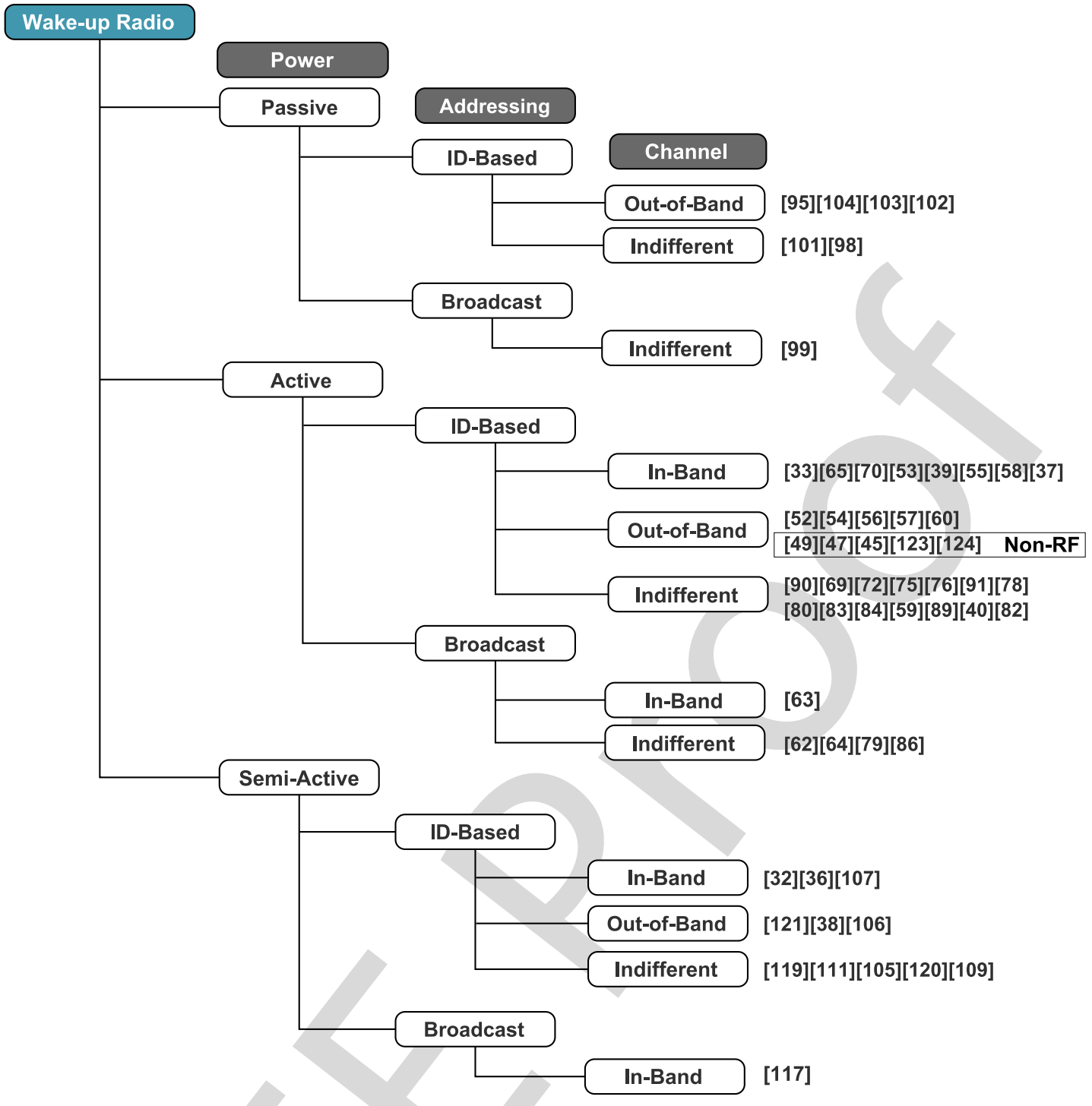

Fig. 4. Taxonomy of wake-up radios showing the hardware design space. 583 explicitly indicate the few systems that are not RF-based, and 584 refer the reader to Table IX for details.

division techniques such as frequency-hopping spread spectrum, this separate channel can further consist of multiple channels to be able to wake-up specific nodes. The benefits of using separate channels for WuS transmission and data include decreased interference from neighboring nodes operating in the same frequency band and increased signal capacity. However, equipping the WuR with separate channel capability may increase the cost and complexity of the system design.

Finally, we look at the different communication mediums that can be utilized for WuS transmission. Fig. 4 does not explicitly show this as a vast majority of the systems we sur-

(i) Medium (RF-Based): If radio signals such as extremely low frequency $(\sim 3 \mathrm{kHz})$ to extremely high frequency (up to several GHz) are used for signaling, the scheme is referred to as RF based wake-up. RF based WuRs have been very widely used and will be discussed in 589 more detail in the next section.

590

(ii) Medium (Acoustic): Acoustic based wake-up such as 591 ultrasonic and audio signals have also been considered. 592 This medium does not require any special infrastructure 593 and the audio signals can be easily generated by speakers 594 or smart phones. Yadav et al. [44], Lattanzi et al. [45], 595 Hoflinger et al. [46], and Sánchez et al. [47] have 596 proposed WuR designs based on sound wave for $\mathrm{WuS}{ }_{597}$ transmission.

598

(iii) Medium (Optical): Optical as a communication medium 599 for WuRs has also be utilized for indoor sensor 600 networks [48], [49]. For example, Mathews et al. [48] 601 have used Free Space Optics (FSO) for sending WuS. 602

As a system designer, this taxonomy serves as a guide to 603 the available WuR technologies that could meet the constraints 604 of the system. Knowing if continuous power can be provided ${ }_{605}$ in a given environment can direct one along the branch with ${ }_{606}$ the appropriate power source. Knowing the approximate node 607 density and the expected data rate can serve as indicators for 608 


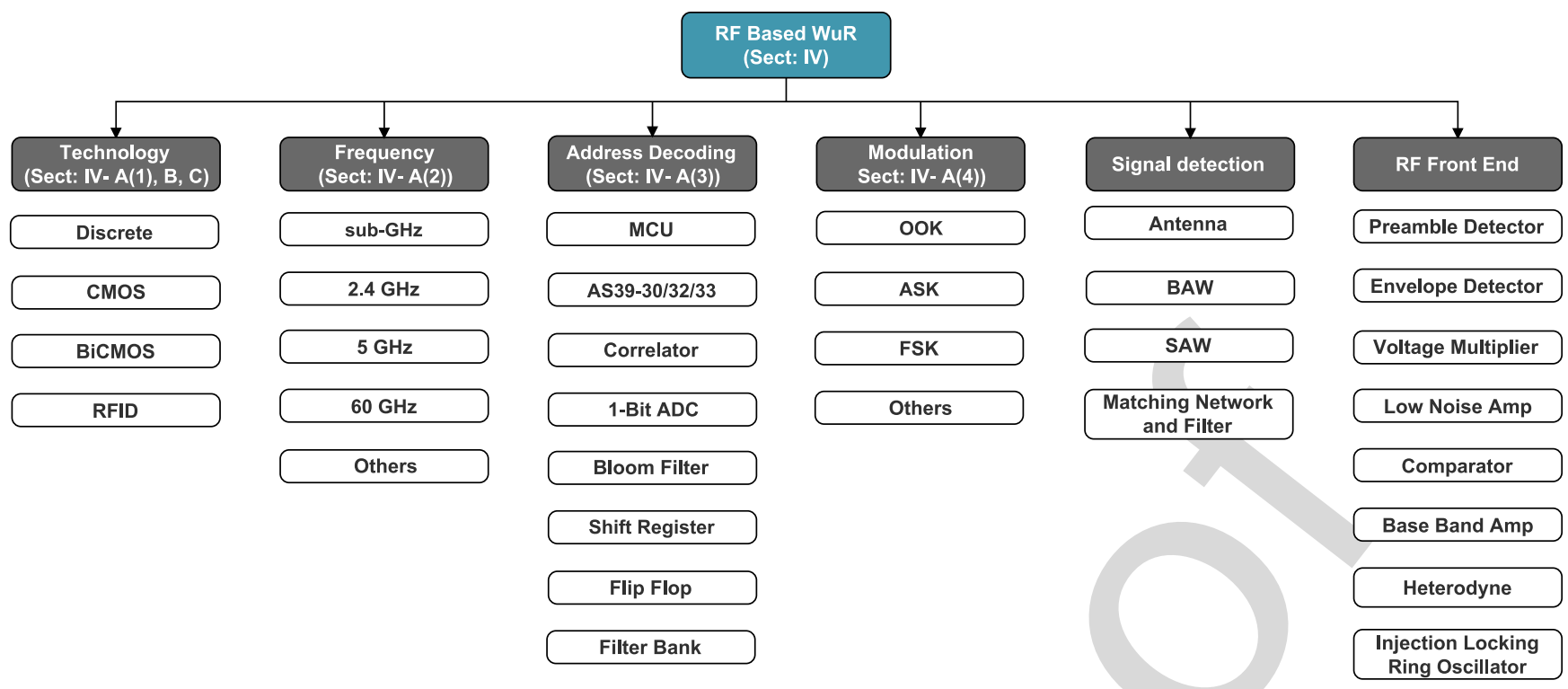

Fig. 5. Defining characteristics of RF-based WuRs with various building blocks. Wake-up radios meeting each characteristic appear in Tables II to VII while Tables VIII and IX provide the full summary of each surveyed prototype.

609 whether unicast, ID-based addressing or broadcast communi610 cation is most appropriate. Finally, the amount of expected 611 data to be transferred can lead one to a solution where the $612 \mathrm{WuS}$ is on a same or different channel.

\section{3 \\ IV. STATE-OF-THE-ART WAKE-Up RAdios}

614 615 focus to the hardware composition of the various prototypes 616 described in the literature. This section offers a comparison 617 of 75 RF-based WuR prototypes, summarized in Table VIII. 618 To offer a clear picture of the current research landscape, we 619 organize this section first along the power source dimension 620 outlined in the previous section: active, passive, and semi621 active systems.

622 Inside our description of active radios, we offer a catego623 rization, overviewed in Fig. 5, that defines the key hardware 624 characteristics. We focus on four: core fabrication tech625 nology, frequency usage, address decoding, and modulation 626 techniques.

627 Following this in-depth discussion of active RF-based WuR, 628 our more concise discussions of passive and semi-active focus 629 on the technology only.

630 Within each subsection we offer a table categorizing the 631 radios of Table VIII according to the options for each fea632 ture, highlighting (in bold and yellow) the prototypes that are 633 described in detail in the text. Not all prototypes appear in 634 each, separate table, as not all information is known about ${ }_{635}$ each prototype, preventing us from adding it to the tables.

${ }_{636}$ We end the section with a brief summary of non-RF WuRs 637 and a discussion.

\section{${ }_{638}$ A. Active Wake-Up Radios}

639 In this section, we present active WuRs that require an exter640 nal current source to receive a packet. In most cases, they 641 are used in an always-on manner, but we defer this usage
TABLE II

WuR CATEGORIZATION BASED ON TECHNOLOGY

\begin{tabular}{|c|c|}
\hline Technology & Reference No. \\
\hline Discrete & $\begin{array}{l}{[33],[50],[51],[52],[39],[53],[54],[55],} \\
{[56],[57],[37],[40],[58],}\end{array}$ \\
\hline CMOS & $\begin{array}{l}\text { [59], [60], [61], [62], [63], [64], [65], } \\
{[66],[67],[68],[69],[70],[71],[72],[73],} \\
{[74],[75],[76],[77],[78],[79],[80],[81],[82],} \\
{[83],[84],[85],[86],[87]}\end{array}$ \\
\hline BiCMOS & {$[88],[89]$} \\
\hline RFID & {$[90]$} \\
\hline
\end{tabular}

discussion to later. As previously mentioned, we divide our 642 discussion of active WuRs into four categories: the technology ${ }_{643}$ used to realize the prototype, operating frequencies that have ${ }_{644}$ been utilized in different bands, address decoding techniques, 645 and wake-up signal modulation.

1) Technology: The overall power consumption of the $\mathrm{WuR} 647$ depends on its design technology as well as its implementa- 648 tion. Mainly, the chip fabrication technology such as CMOS 649 and BiCMOS for digital circuits and the use of off-the-shelf 650 discrete components for analog circuitry. Although off-the- 651 shelf components allow quick implementation, CMOS based ${ }_{652}$ WuRs are more energy efficient and have smaller form factors. 653

Use of off-the-shelf discrete components and IC packages 654 has allowed designers to simplify and foster rapid prototyp- 655 ing of WuRs with low power consumption, low cost, ease of 656 changes, and reliability.

Petrioli et al. [39] presented a WuRx using fully discrete 658 components that support four different channels in a $2.4 \mathrm{GHz} 659$ band, thus enabling node addressing. The receiver front end 660 consists of the antenna, low noise amplifier and three power ${ }_{661}$ slitters followed by the filter bank. According to the tests, the 662 sensitivity of the WuRx is $-83 \mathrm{dBm}$, while its power con- ${ }_{663}$ sumption is $1620 \mu \mathrm{W}$. The line-of-sight communication range ${ }_{664}$ 


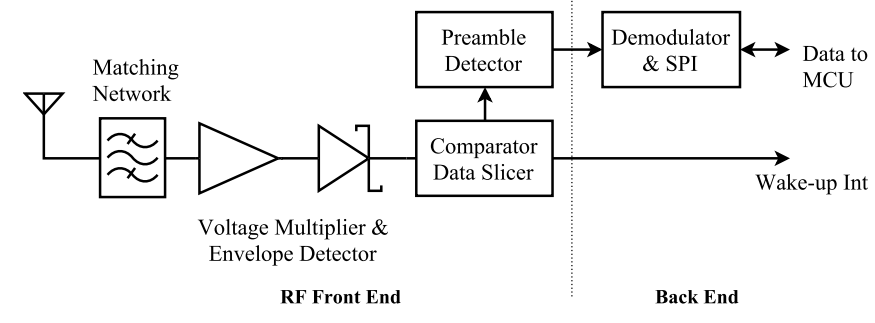

Fig. 6. Discrete components based WuRx architecture [40].

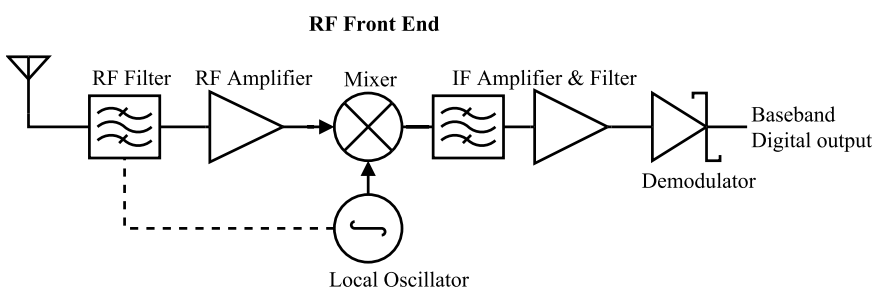

Fig. 7. Generic Block diagram of Heterodyne WuRx [62].

665 is $120 \mathrm{~m}$, the highest range attained using low complexity 666 receiver design. However, this design also has higher power 667 demand compared to other WuRxs in this category and does 668 not provide the details for the transmission power required to 669 achieve this range.

670 In recent years power consumption of CMOS devices has 671 greatly reduced allowing researchers to design ultra-low power 672 circuits. There are $29 \mathrm{WuR}$ prototypes based on CMOS 673 technology.

674 In chronological order, the idea of developing and using 675 ultra-low power radios as WuRs was first conceived by the 676 PicoRadio project [59], which proposed a CMOS based node 677 architecture that could be used both as a data radio and as a $678 \mathrm{WuR}$ using a carrier frequency of $1.9 \mathrm{GHz}$ with data rate up 679 to $100 \mathrm{kbps}$. The PicoRadio has a $10 \mathrm{~m}$ range and consumes 680 around $380 \mu \mathrm{W}$ from a supply voltage of $1 \mathrm{~V}$. However, not 681 much detail was provided on the hardware side.

682 Many of the proposed CMOS based prototypes have 683 adopted a heterodyne approach. Heterodyne is a method to 684 convert an incoming high frequency RF signal into one at 685 a lower frequency by mixing two or more signals, where 686 high gain and selectivity could be obtained with relative ease 687 (Fig. 7).

${ }_{688}$ Pletcher et al. [60] proposed a $1.9 \mathrm{GHz}$ WuRx chip consum689 ing $65 \mu \mathrm{W}$ from a $0.5 \mathrm{~V}$ supply in an active mode (receiving 690 and decoding the $\mathrm{WuS}$ ). The receiver data rate and the sensi691 tivity are $40 \mathrm{kbps}$ and $-50 \mathrm{dBm}$, respectively using OOK for $692 \mathrm{WuS}$ modulation. The design was further improved in [62] 693 by using an "uncertain-IF" architecture to reduce the power 694 consumption to $52 \mu \mathrm{W}$ with enhanced data rate and sen695 sitivity of $100 \mathrm{kbps}$ and $-72 \mathrm{dBm}$, respectively. The WuRx 696 consists of BAW resonator for network impedance matching, 697 a front-end-IF (Intermediate Frequency) amplifier for RF sig698 nal conditioning and amplification followed by an envelope 699 detector for extracting the shape of the signal and converting 700 it to direct current (DC) for triggering the node's MCU.
A simulation based super-regenerative heterodyne WuRx 701 using duty cycling scheme is proposed by $\mathrm{Yu}$ et al. [61]. 702 The super-regenerative WuRx consists of an isolation amplifier 703 as an interface between the antenna and oscillator providing 704 network matching followed by an envelope detector. To reduce 705 power consumption, the oscillator is duty cycled at 10\%. With 706 duty cycling, the WuRx dissipates an average power of $56 \mu \mathrm{W} 707$ in listening mode for $100 \mathrm{kbps}$ OOK modulated signal using 708 $2.4 \mathrm{GHz}$ carrier frequency. However, this power consump- 709 tion increases drastically to $525.6 \mu \mathrm{W}$ at $1.8 \mathrm{~V}$ supply if 710 no duty cycling is applied. Similarly, the WuRx prototype 711 presented by Yoon et al. [70] also employs duty cycling. The 712 proposed WuRx features two modes of operation; monitor- 713 ing mode (MO) for receiving the preamble and identification 714 mode (ID) for node address decoding. The WuRx is only duty 715 cycled in the MO mode while in the ID mode the duty cycling 716 is terminated and the data is received at higher data rate. In 717 MO mode this node consumes as low as $8.4 \mu \mathrm{W}$ from a $1.8 \mathrm{~V} 718$ power supply offering a data rate of $1 \mathrm{kbps}$. As a consequence 719 of high bit rate of $200 \mathrm{kbps}$ employed for address decoding, 720 the power surges to $1100 \mu \mathrm{W}$ for the receiver sensitivity of 721 $-73 \mathrm{dBm}$.

722

Another $2.4 \mathrm{GHz}$ based heterodyne WuRx was proposed by 723 Drago et al. [65]. The WuS is modulated using pulse-position- 724 modulated (PPM) impulse radio modulation scheme. The main 725 building blocks of this WuRx front end are an antenna, a 726 matching network with an on-chip inductor, and a local- 727 oscillator (LO) generator for down-converting the frequency. ${ }^{728}$ This IF signal is then amplified using multiple frequency IF- 729 amplifier and then down-converted to baseband by a full-wave 730 rectifier. To achieve low power consumption, the receiver front ${ }_{731}$ end as well as the LO generator are duty-cycled at pulse level, 732 thereby reducing the power consumption to $415 \mu \mathrm{W}$. The full ${ }_{733}$ WuRx prototype achieves a sensitivity of $-82 \mathrm{dBm}$ at a data 734 rate of $500 \mathrm{~kb} / \mathrm{s}$ with energy efficiency of $830 \mathrm{pJ} / \mathrm{bit}$. 735

There are also designs reported in the literature with power 736 consumption above $1000 \mu \mathrm{W}$ [64], [75], [87], [89] com- 737 pared to the ones discussed earlier. The WuRx proposed by 738 Bdiri and Faouzi [87] has attained the longest communica- ${ }_{739}$ tion range of $82 \mathrm{~m}$ using heterodyne approach at transmission 740 power of $10 \mathrm{dBm}$ with receiver sensitivity of $-60 \mathrm{dBm}$. 741 However, at the same time this particular WuRx has the high- 742 est power demand of $5247.5 \mu \mathrm{W}$ when receiving and decoding 743 the WuS. Other heterodyne based WuRx prototypes achieving 744 power consumption between $22 \mu \mathrm{W}$ and $100 \mu \mathrm{W}$ have also 745 been reported in [77], [78], [81], and [84].

Radio-Frequency Identification (RFID) technologies have 747 been used as WuR for accomplishing asynchronous multi- 748 modal wake-up where an off-the-shelf RFID tag and an RFID 749 reader has been utilized as a WuRx and WuTx, respectively. 750 Fig. 10 illustrates a simple architecture for utilizing RFID ${ }_{751}$ technology for WuR systems.

An off-the-shelf active RFID tag based WuRx is simulated 753 in [90]. RFIDImpulse uses an RFID reader as a WuTx to 754 trigger an RFID tag that is attached to a remote sensor node at 755 an operational distance of up to $30 \mathrm{~m}$ while consuming $80 \mu \mathrm{W} \quad{ }_{756}$ of power. However, this receiver does not utilize addressing 757 to selectively wake up a sensor node. 
TABLE III

WUR CATEGorizATION BASED ON FREQUENCY USAGE

\begin{tabular}{|l|l|}
\hline Band & Reference No. \\
\hline sub-GHz & $\begin{array}{l}{[85],[37],[33],[67],[50],[74],[51],[52],} \\
{[53],[54],[55],[40][70],[81],[64],[78],} \\
{[63],[57],[80]}\end{array}$ \\
\hline $2.4 \mathrm{GHz}$ & $\begin{array}{l}{[88],[61],[65],[90],[68],[73],[75],[39],[89],} \\
{[77],[56],[82],[83],[84],[80]}\end{array}$ \\
\hline $5 \mathrm{GHz}$ & {$[\mathbf{7 9}]$} \\
\hline $60 \mathrm{GHz}$ & {$[\mathbf{6 9}],[\mathbf{7 2}]$} \\
\hline Others & {$[\mathbf{5 8}],[\mathbf{7 1}],[86],[87],[59],[60],[62]$} \\
\hline
\end{tabular}

759 2) Operating Frequency: Another layer of complexity is 760 added when considering the transmission frequency of the ${ }_{761} \mathrm{WuR}$. Further, if the WuR and the main data transceiver 762 are using different frequencies, each requires a separate 763 antenna for signal detection and separate matching networks. 764 Moreover, the choice of the operating frequency for WuRx 765 is critical as it determines the size of the antenna and the 766 operational range of the system as a whole.

767 The sub-GHz WuRx presented by Spenza et al. [54] con768 sumes $1.276 \mu \mathrm{W}$ in listening mode. The receiver uses OOK 769 modulation and is made of four main building blocks: a match770 ing network, an envelope detector followed by a comparator 771 and a preamble detector. At the receiver end, the output from 772 the preamble detector is used to interrupt an on-board 8-bit 773 PIC12LF1552 MCU that performs address matching and trig774 gers the main sensor node when a valid wake-up address is 775 received. This sub-GHz WuRx provides high sensitivity and 776 data rate of $-55 \mathrm{dBm}$ and $100 \mathrm{kbps}$ respectively, while achiev777 ing the maximum wake-up range of $45 \mathrm{~m}$. This design is 778 further improved by Magno et al. [40], which achieves power 779 consumption in listening mode of $0.152 \mu \mathrm{W}$ at $32 \mathrm{dBm}$ sensi780 tivity and $1.196 \mu \mathrm{W}$ for the $-55 \mathrm{dBm}$ version. This particular 781 WuRx has achieved an interesting communication range of up 782 to $50 \mathrm{~m}$ and offers data rate of $10 \mathrm{kbps}$.

783 Multi-band WuRs have also been exploited to increase 784 the flexibility and to allow interoperability between differ785 ent frequencies used in WSNs. Robert et al. [57] propose 786 an ultra-low power WuRx for indoor/outdoor asset tracking 787 systems that consumes only $5 \mu \mathrm{W}$. Authors have developed a 788 tag module that contains a transmitter and two WuRxs inte789 grated in one module. The $434 \mathrm{MHz}$ WuRx is intended for 790 indoor localization, and the $868 \mathrm{MHz}$ WuRx and transmitter 791 are used for the data exchange with the gateways for outdoor 792 localization. The WuRx continuously scans the channel for 793 any predefined wake-up sequences. As soon as the received 794 sequences matches to the reference sequence, a digital control 795 signal is generated immediately to trigger the sensor node. 796 In addition, the proposed WuRx also provides a received sig797 nal strength indicator (RSSI) value of the received WuS with 7983 bits quantization. A similar prototype for asset tracking 799 applications has also been reported in [80]. The Fraunhofer 800 WakeUp-Receiver [80], which is based on 130-nm CMOS 801 technology, operates in the $868 \mathrm{MHz}$ and $2.4 \mathrm{GHz}$ frequency 802 bands and features $-80 \mathrm{dBm}$ sensitivity with 16-bit selective
TABLE IV

WUR FEATURING ADDRESS DECODING

\begin{tabular}{|l|c|}
\hline Technique & Reference No. \\
\hline MCU & {$[58],[33],[52],[54],[55],[40],[73]$} \\
\hline Correlator & $\begin{array}{c}{[67],[74],[78],[88],[68],[82],[76],} \\
{[89]}\end{array}$ \\
\hline AS3930 & {$[37],[50],[51],[53],[87],[56]$} \\
\hline Others & {$[70],[63],[81],[39]$} \\
\hline
\end{tabular}

wake-up ID. At a data rate of $1 \mathrm{kbps}$ this prototype consumes 803 $7.5 \mu \mathrm{W}$ of power with response time of $30.3 \mathrm{~ms}$. However, 804 no detailed operational communication range tests or complete 805 WuR system design is provided.

806

To achieve relatively high date rates, a WuRx operating in 807 millimeter-wave band $(60 \mathrm{GHz})$ for short-range applications 808 is proposed in [69]. This duty cycled WuR consists of a 4- 809 path phase array transmitter and a 4-path receiver. By applying 810 OOK modulation for switching the biasing of power ampli- 811 fiers a $1 \mathrm{Gbps}$ data rate is attained. The WuRx side is built of 812 an injection-locking ring oscillator (ILRO), a frequency mixer 813 and a low pass filter. The performance of this receiver is eval- 814 uated in simulations and has achieved a power consumption 815 of $230 \mu \mathrm{W}$ with sensitivity of $-62 \mathrm{dBm}$ ranging up to $0.2 \mathrm{~m}$. 816 Instead, Wada et al. [72] presented a first successful WuRx 817 prototype operating at $60 \mathrm{GHz}$. To achieve low power con- 818 sumption, a power reduction circuit has been implemented 819 that turns off the injection locking oscillator when there is 820 no WuS detected. The fabricated WuRx has a high sensitivity 821 of $-68 \mathrm{dBm}$ for a $350 \mathrm{kbps}$ OOK WuS while consuming only 822 $9 \mu \mathrm{W}$ from a $1.5 \mathrm{~V}$ supply. Another WuRx that operates at 823 $5.8 \mathrm{GHz}$ has been reported in [79] but has lower sensitivity 824 of $-44 \mathrm{dBm}$. Note that for the latter two designs, the authors 825 have not published any operational distance. 826

There are also few WuR designs for WBAN applications 827 that use very low frequency for communication. One of the 828 advantages of operating at lower frequency bands is that it 829 enables lower signal attenuation and interference than the tra- 830 ditional operating bands such as $2.4 \mathrm{GHz}$. Cho et al. [71] 831 proposed the WuRx prototype targeting WBAN applications 832 while operating at $45 \mathrm{MHz}$. The proposed WuRx uses ILRO 8 instead of RF amplifier to reduce power consumption. The 834 $\mathrm{WuS}$ is modulated using Frequency Shift Keying (FSK) and 835 is demodulated by a low power Phase Locked Loop (PLL) 836 demodulator. This prototype features a receiver sensitivity of 837 $-62.7 \mathrm{dBm}$ with data rate of $200 \mathrm{kbps}$ while consuming as low 838 as $37.5 \mu \mathrm{W}$ from a $0.7 \mathrm{~V}$ supply in an active mode. 8 .

Recently, Petäjäjärvi et al. [58] proposed a $28 \mathrm{MHz} 840$ always on WuR design based on super-regenerative princi- 841 ple for human body communications. To achieve low energy 842 consumption and high sensitivity, the WuR uses loose syn- 843 chronization and employs self-quenching while operating at 844 $1.25 \mathrm{kbps}$. With real-life experiments the proposed designed 845 consumes $40 \mu \mathrm{W}$ and achieved receiver sensitivity of $-97 \mathrm{dBm} .846$

3) Address Decoding: Next, adding node address decoding 847 capability to the WuRx requires additional components at the 848 RF back-end. Usually, a low power micro-controller (MCU) 849 


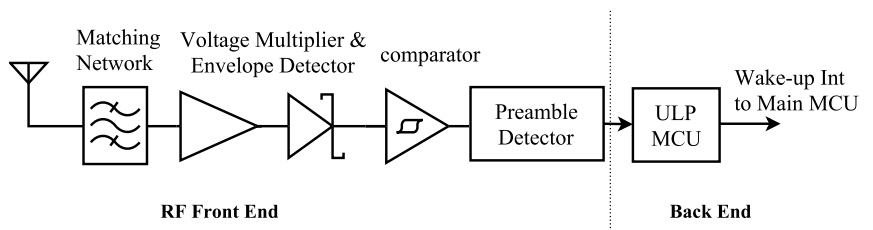

Fig. 8. Wake-up receiver employing an ultra-low power MCU for address decoding and interrupt generation.

850 or correlator is employed for decoding. However, this comes 851 with some trade-offs, highlighted in this section.

${ }_{852}$ Some WuR designs use a secondary, dedicated low-power 853 micro-controller to decode the address code. An example is 854 shown in Fig. 8, illustrating the integration of low power 855 MCUs with WuR prototypes. As will be discussed later, this 856 extra hardware contributes to energy overhead when used for 857 address decoding.

858 Using a separate MCU for address decoding and 859 interference filtering is reported in [33]. In this prototype, 860 authors have integrated a PIC12F683 MCU to detect and 861 decode a WuS after signal rectification and amplification, 862 and notify the more powerful AT-mega128L processor of the 863 main node through an interrupt. Due to intervention of this 864 extra PIC12F683 MCU, the overall power consumption of the $865 \mathrm{WuRx}$ increases from $171 \mu \mathrm{W}$ in listening mode to $819 \mu \mathrm{W}$ 866 at $3 \mathrm{~V}$ when used for address decoding. The proposed proto867 type was only able to communicate up to $2 \mathrm{~m}$ with receiver 868 sensitivity of $-51 \mathrm{dBm}$ at data rate of $0.86 \mathrm{kbps}$ using $\mathrm{OOK}$ 869 modulation. Another prototype with similar communication 870 range is presented by Bdiri and Derbel [55], but has lower 871 power consumption of $0.69 \mu \mathrm{W}$ operating in $868 \mathrm{MHz}$ band. 872 Authors have also compared two different WuS decoding tech873 niques, one with MCU and the other using AS3932 (a detailed 874 discussion of the AS323X series will follow at the end of this 875 subsection). The results indicate that using AS3932 for address 876 decoding leads to an additional power consumption of $3.9 \mu \mathrm{W}$ 877 than the MCU.

878 Other designs that exploit MCU for address decoding while 879 achieving power consumption below $15 \mu \mathrm{W}$ can be found 880 in [52] and [73]. However, these designs do not provide any 881 detail on operational distance that can be achieved with these 882 WuRxs.

883 Instead of using MCUs for address decoding, an energy 884 efficient way is to use correlator circuit for address match$885 \mathrm{ing}$. In the correlator circuit, the node address is stored in 886 the reference signal buffer and the input bits from the WuS 887 are correlated against the reference signal. When a new bit is 888 available, all the samples are shifted one position in the cor889 relator and are compared to the pre-stored one. If the stored 890 and the incoming bits are a match, the wake-up interrupt pin is 891 asserted. Fig. 9 depicts a simple "matched filter" based parallel 892 correlator concept used to decode address in a WuS.

893 Von der Mark and Boeck [88] simulated one of the first 894 correlator based approaches for decoding node address in a $895 \mathrm{WuRx}$ system and features sensitivity of $-50 \mathrm{dBm}$. The wake896 up circuit is composed of a $2.4 \mathrm{GHz}$ matching network, an 897 envelope detector, and low noise amplifier. The output signal

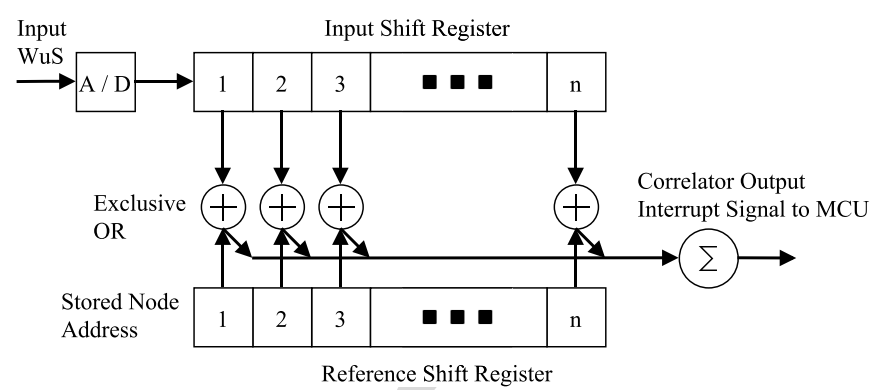

Fig. 9. Node address comparison using "matched filter" correlation detector.

from the amplifier is then fed into the correlator circuit to 898 compare the signal to a predefined sequence. However, no 899 values have been reported for power consumption, data rate 900 or WuRx communication range.

901

Hambeck et al. [67] presented a complete prototype of 902 WuRx employing a 64-bit mixed signal correlator for address 903 matching. At $868 \mathrm{MHz}$, the design features a receiver sen- 904 sitivity of $-71 \mathrm{dBm}$ and an outstanding measured free-space 905 radio link distance of up to $304 \mathrm{~m}$ at transmission power of 906 $6.4 \mathrm{dBm}$. At this conditions, the WuRx dissipates only $2.4 \mu \mathrm{W} 907$ at supply voltage of $1 \mathrm{~V}$.

908

Milosiu et al. [74] presented a 31-bit correlator based WuRx 909 with scalable data rate and $-83 \mathrm{dBm}$ sensitivity. The proto- 910 type is fabricated in a 130-nm CMOS technology and requires 911 $4.75 \mu \mathrm{W}$ from a $2.5 \mathrm{~V}$ supply at a data rate of $128 \mathrm{bps}$. 912 Compared to the other WuRx prototypes found so far in the 913 literature, the proposed receiver has obtained the longest line- 914 of-sight communication range of $1200 \mathrm{~m}$ for a transmit power 915 of $10 \mathrm{~mW}$. Recently, authors have also proposed a $2.4 \mathrm{GHz} 916$ version of the OOK WuRx that obtains a power consumption 917 of $7.25 \mu \mathrm{W}$ with reaction time of $30 \mathrm{~ms}$. However, no details 918 on the receiver range is provided. Other low power designs 919 have also been reported in [68] obtaining power consumption 920 below $3 \mu \mathrm{W}$.

There are many proposals in the literature where 922 authors have also resorted to a commercially avail- 923 able WuRx chip for address decoding into their 924 prototypes [36], [37], [51], [53], [55], [87]. The AS393X 925 series from Austria Microsystems [91] is a 3D low-power 926 low-frequency Amplitude Shift Keying (ASK) WuRx capable 927 of generating a wake-up interrupt upon detection of signal at ${ }_{928}$ a carrier frequency between $15-150 \mathrm{kHz}$. The AS393X also 929 allows duty cycling the WuRx in order to save energy and 930 includes an integrated correlator to implement a 16 bit or ${ }_{931}$ 32 bit wake-up address decoding scheme. This WuRx has 932 maximum sensitivity of $-69 \mathrm{dBm}$ with current consumption 933 varying from $1.7 \mu \mathrm{A}$ up to $12 \mu \mathrm{A}$ at $3 \mathrm{~V}$ power supply. With ${ }_{934}$ these characteristics, the AS393X has average performance 935 compared to other experimental WuR prototypes found in the 936 literature.

937

Sutton et al. [37] presented the first practical application 938 of WuRx that can be used both for initiating the communi- 939 cation and as a full data radio. The OOK WuR transceiver 940 is designed using the off-the-shelf components and leverages 941 AS3930 ASK receiver for address decoding. The CC110L 942 
TABLE V

Various WuS MOdulation TeChNiques

\begin{tabular}{|l|l|}
\hline Modulation & Reference No. \\
\hline OOK & {$[86],[85],[59],[60],[62],[61],[75],[77]$,} \\
& {$[83],[69],[72],[66],[70],[37],[50],[51]$,} \\
& {$[53],[87],[56],[74],[88],[68],[82],[76]$,} \\
& {$[89],[39],[58],[33],[54],[55],[40],[73]$,} \\
& {$[81],[84],[67]$} \\
\hline ASK & {$[90],[79],[63],[52]$} \\
\hline FSK & {$[71],[64],[78],[84]$} \\
\hline Others & {$[65]$} \\
\hline
\end{tabular}

943 transceiver is used as a WuTx and shares the same antenna 944 with the WuRx module. The OOK receiver is able to receive 945 a 16-bit data packet at a maximum data rate of $8.192 \mathrm{kbps}$, 946 and features an ultra-low power consumption of $8.1 \mu \mathrm{W}$ mea947 sured at $3 \mathrm{~V}$. The OOK receiver sensitivity is approximately $948-52 \mathrm{dBm}$ and achieves a $30 \mathrm{~m}$ line-of-sight communication 949 range in an outdoor field.

950 Oller et al. [53] proposed WuRx incorporating AS3933 951 for IEEE802.11-enabled wireless access points. This proto952 type features a WuRx sensitivity of $-52 \mathrm{dBm}$ and the total ${ }_{953}$ power consumed by the design is $10.8 \mu \mathrm{W}$ in sleep mode 954 and $24 \mu \mathrm{W}$ in an active mode with address decoding. Similar 955 wake-up range of up to $40 \mathrm{~m}$ has been observed making these 956 prototypes suitable for implementation that require long range 957 communication with minimum power consumption without 958 relying on MCU for address decoding.

959 Microsemi based ZL70103 [92] is another off-the-shelf 960 transceiver chip that incorporates a WuRx designed for 961 implantable medical devices. The out-of-band WuRx operates 962 at $2.45 \mathrm{GHz}$ with an average current consumption of $290 \mathrm{nA}$ 963 while sniffing the channel once a second. It allows to initi964 ate the communication between the implanted device and the 965 base station transceiver using specially coded WuS from the $9662.45 \mathrm{GHz}$ base station. So far, none of the prototypes presented 967 in this survey use ZL70103, however it is an interesting option 968 for BAN applications.

969 Other address decoding techniques using Bloom filters [63], 970 shift registers [81], flip-flops, and filter banks [39] have also 971 been exploited. Takiguchi et al. [63] have simulated a Bloom 972 filter based wakeup mechanism for WuRxs. A node identifier973 matching mechanism uses Bloom filter implemented with a 974 simple circuit that only uses an AND circuit. For a bit rate 975 of $40 \mathrm{kbps}$, the listening power consumption of the receiver is $97612.4 \mu \mathrm{W}$ and in an active state the circuit consumes $368.1 \mu \mathrm{W}$ 977 from a $1.8 \mathrm{~V}$ supply.

978 4) WuS Modulation Technique: Circuit complexity and 979 reproducibility are the key factors that allow designers to tune 980 and simplify WuRs enabling faster prototyping. Nevertheless, 981 this is dependent on the modulation technique used for $\mathrm{WuS}$ 982 transmission, the architecture of RF front- and back-end, and ${ }_{983}$ the choice of frequency. To meet the requirement of ultra984 low power consumption, various modulation schemes such 985 as on/off keying (OOK), Amplitude shift keying (ASK), or ${ }_{986}$ Frequency shift keying (FSK) have been exploited for the wake 987 up signals.
TABLE VI

Technology Utilized for Passive WuR

\begin{tabular}{|l|c|}
\hline Technology & Reference No. \\
\hline Discrete & {$[\mathbf{9 3}]$} \\
\hline CMOS & {$[94],[\mathbf{9 5}],[96],[\mathbf{9 7}],[\mathbf{9 8}],[99]$} \\
\hline RFID & {$[\mathbf{1 0 0}],[\mathbf{1 0 1}],[\mathbf{1 0 2}]$} \\
\hline
\end{tabular}

As seen from Table $\mathrm{V}$, most of the WuR designs have mod- 988 ulated RF signal using OOK before reception by the wake-up 989 receiver. In OOK modulation scheme the signal information is 990 delivered using ' 1 's or ' 0 's. The source node transmits a large 991 amplitude carrier when it wants to send a ' 1 ' and nothing is 992 send for ' 0 ', i.e., the transmitter is turned off. Thus, allowing ${ }_{993}$ systems to save on transmit power when (not) sending '0's. 994 On the receiver side this signal is sensed by the rising edge 995 of the digital signal from low to high indicating that a valid 996 signal has been received via the antenna. This has enabled 997 OOK hardware implementations to be relatively straight- 998 forward due to their low implementation cost for battery- 999 operated applications. Usually, few discrete components are 1000 enough to construct OOK signal detection circuitry as out- 1001 lined in [38] and [54]. The super-generative [71], [72], tuned 1002 RF [40], [53], [59], or uncertain-IF architectures [75], [89] 1003 have been popular solutions to demodulate an OOK signal. 1004 In [40], the WuRx consumed $1.2 \mu \mathrm{W}$ and achieved a sensi- 1005 tivity of $-55 \mathrm{dBm}$ at a data rate of $10 \mathrm{kbps}$ to demodulate a 1006 $868 \mathrm{MHz}$ OOK signal.

1007

ASK is another popular modulation technique used by WuR 1008 hardware designers. Similar to OOK, the information in ASK 1009 is also transmitted using ' 1 's or ' 0 's. However, instead of keep- 1010 ing the transmitter off when indicating bit ' 0 ', it transmits 1011 small amplitude carrier in its simplest form. 1012

For FSK demodulation, WuRxs are based on frequency 1013 discrimination architecture. In [64], the WuRx consumes 1014 $2700 \mu \mathrm{W}$ to demodulate a $0.915 \mathrm{MHz}$ FSK signal. The overall 1015 receiver sensitivity is $-89 \mathrm{dBm}$ at a data rate of $45 \mathrm{kbps}$. 1016

Most of the designs surveyed in this paper are compati- 1017 ble with only one modulation technique. Therefore, to make a 1018 WuRx compatible with other types of signals, Taris et al. [84] 1019 proposed a first dual modulation based WuRx. This proof of 1020 concept features an LC oscillator coupled with an envelope 1021 detector implemented in a $65 \mathrm{~nm}$ CMOS technology. The 1022 circuit consumes $120 \mu \mathrm{W}$, and properly demodulates $\mathrm{OOK}_{1023}$ and FSK modulated signals at $2.4 \mathrm{GHz}$ with data rate up to 1024 500 kbps.

1025

Although, ASK offers better noise immunity compared to 1026 OOK at a lower cost than FSK, it has higher power consump- 1027 tion demand than OOK based WuRxs (refer to Table VIII and ${ }_{1028}$ Fig. 13).

1029

\section{B. Passive Wake-Up Radios}

1030

This section discusses prototypes that harvest and power ${ }_{1031}$ the wake-up circuitry entirely from the RF signal. In this 1032 way, passive WuRxs have the advantage of not consuming ${ }_{1033}$ any energy from the node battery making the design energy 1034 neutral. 
1036 The first proof-of-concept passive WuRx design operat1037 ing at a frequency of $433 \mathrm{MHz}$ was presented by $\mathrm{Gu}$ and 1038 Stankovic in 2005 [93]. The WuRx is powered using radio 1039 signals and is able to trigger a wake-up interrupt once enough 1040 energy has been harvested and stored on the capacitor. The 1041 proposed WuRx uses a charge pump approach consisting of 1042 capacitors and zero-bias Schottky diodes acting as a voltage 1043 multiplier and a radio trigger circuit. This WuRx also features 1044 the addressing capability by transmitting the WuS at different 1045 frequencies to activate the targeted node, reaching an operating 1046 range of around $3 \mathrm{~m}$. The power consumption of the WuRx 1047 in idle mode (i.e., while harvesting energy from the WuS) is $1048145 \mu \mathrm{W}$, and the design was only evaluated through SPICE 1049 circuit simulations.

1050 Another battery-less WuRx operating at $900 \mathrm{MHz}$ band was 1051 proposed in [95]. This passive CMOS chip consists of an RF 1052 front end and a digital baseband with non volatile memory. ${ }_{1053}$ The radio block includes a voltage multiplier for rectifying 1054 and amplifying the RF energy, a voltage limiter, demodulator 1055 and modulator circuits, and a ring oscillator. Authors have 1056 designed the voltage multiplier by cascading 4-stage voltage 1057 doublers using Schottky diodes and capacitors. Using ASK 1058 modulation technique, the prototype achieved a sensitivity of $1059-17 \mathrm{dBm}$ with power consumption of $2.64 \mu \mathrm{W}$. However, no 1060 details regarding the communication range and data rate are 1061 provided.

1062 Kamalinejad et al. [97] presented a passive $868 \mathrm{MHz}$ WuRx 1063 front end that also harvests energy from the RF signal. The 1064 building blocks consist of an antenna, matching network, 1065 voltage multiplier and data slicer (comparator and the refer1066 ence generator). An RF-to-DC converter is used to produce 1067 the envelope of the OOK WuS and converts the RF signal 1068 to a DC voltage that is used to power the data slicer cir1069 cuitry. A fraction of this DC output is then compared with the 1070 generated reference to produce the wake-up interrupt signal. 1071 Using simulations, the proposed design exhibits a sensitivity 1072 of $-33 \mathrm{dBm}$ and $100 \mathrm{kbps}$ data rate without any node address1073 ing capability. In turn, Zgaren et al. [98] took the idea of 1074 Kamalinejad et al. [97] and have proposed a passive WuRx 1075 prototype for implantable devices operating in $902-925 \mathrm{MHz}$ 1076 band. This prototype has a power dissipation of $0.2 \mu \mathrm{W}$ for 1077 a data rate of $100 \mathrm{kbps}$ at $-53 \mathrm{dBm}$ sensitivity. However, the 1078 latter design is only evaluated using simulations. Other pas1079 sive WuRxs that are based on CMOS technology can be found 1080 in [94], [96], and [99]

1081 Ba et al. [102] proposed a passive RFID device called 1082 WISP-Mote by combining a Wireless Identification and 1083 Sensing Platform (WISP) to a Tmote Sky sensor node. WISP 1084 is powered wirelessly by an off-the-shelf UHF RFID reader 1085 to generate an external interrupt to a Tmote Sky, achieving 1086 communication range of up to $5 \mathrm{~m}$. Upon successful activa1087 tion, WISP transmits the sensor data using the main node's $10882.4 \mathrm{GHz}$ CC2420 transceiver. WISP supports both broadcast 1089 and ID-based wake-ups.

1090 Passive RFID based systems usually have a communi1091 cation range only up to few meters, thus making it diffi1092 cult to implement a multi-hop sensor network. Therefore, 1093 to realize a multi-hop wake-up using RFID technology,
TABLE VII

SEMI-ACTIVE WuR DESIGNS

\begin{tabular}{|c|c|}
\hline Technology & Reference No. \\
\hline Discrete & $\begin{array}{l}{[\mathbf{3 6}],[103],[104],[\mathbf{1 0 5}],[38],[106],} \\
{[107]}\end{array}$ \\
\hline CMOS & $\begin{array}{l}{[108],[109],[110],[\mathbf{1 1 1}],[112],[113],} \\
{[114],[\mathbf{1 1 5}],[\mathbf{1 1 6}],[117],[118],[\mathbf{3 2}]}\end{array}$ \\
\hline RFID & [119] \\
\hline
\end{tabular}

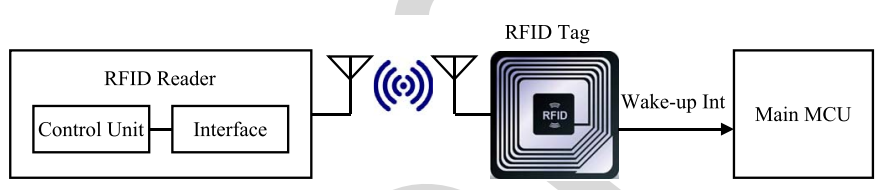

Fig. 10. RFID-based Wake-up receiver prototype [102].

Chen et al. [100] proposed an enhanced version of WISP- 1094 Mote with energy harvesting capabilities called Multi-hop- 1095 Range EnhAnCing energy Harvester-Mote (MH-REACH- 1096 Mote). MH-REACH-Mote is equipped with both a WuTx 1097 and a passive WuRx. The WuRx side is same as WISP- 1098 Mote while UHF RFID reader has been used as the WuTx 1099 providing an option for an addressable wake-up with high 1100 transmission power. This prototype achieved the maximum 1101 wake-up range of $9.4 \mathrm{~m}$ when the WuS was transmitted for 1102 10s. Donno et al. [101] also proposed a passive WuRx pro- 1103 totype using commercial $868 \mathrm{MHz}$ UHF RFID tag and RFID 1104 energy harvester for achieving long distances. Authors imple- 1105 mented a wake-up strategy called Enhanced Write Wake Up 1106 (E-WWU) that supports both broadcast communication and 1107 node addressing achieving a range of $22 \mathrm{~m}$ with transmis- 1108 sion power of $30 \mathrm{dBm}$. The WuRx side consumes $54 \mu \mathrm{W}$ for 1109 receiving and decoding the $\mathrm{WuS}$.

1110

From the above designs, it is evident that CMOS technology 1111 is more popular for implementing passive WuRxs due to its 1112 low power consumption. RFID has also been utilized since it 1113 already provides energy harvesting capabilities thus reducing 1114 the implementation time.

\section{Semi-Active Wake-Up Radios}

To operate in the nano-Watt power range, the majority of 1117 the proposed design approaches are semi-active, in which 1118 only a few components of the receiver front-end are battery 1119 powered while the rest of the components are fully passive. 1120 Using passive circuitry allows reducing the power consump- 1121 tion, but at the price of short communication range and reduced 1122 receiver sensitivities. For the radio front-end, the most com- 1123 mon approach is to implement an envelope detector using 1124 passive components such as Schottky diodes, MOSFETs or 1125 ICs followed by the active components such as correlators and 1126 comparators to generate an interrupt to the main MCU. Next, 1127 we present WuR prototypes that utilize such architecture. ${ }_{1128}$

Malinowski et al. [119] reported the first "quasi-passive ${ }_{1129}$ wake-up" system utilizing RFID technology called CargoNet. 1130 CargoNet employs a $300 \mathrm{MHz}$ RFID tag to trigger an ultra-low 1131 power MSP430 based sensor node. The WuS detector circuit 1132 
1133 consists of an LC tank with an autotransformer for amplify1134 ing the signal received at the antenna followed by an envelope 1135 detector and micro-power amplifier for voltage gain. After the 1136 main sensor node is activated, data is communicated using 1137 a $2.4 \mathrm{GHz}$ CC2500 transceiver. The proposed WuRx design 1138 consumes $2.8 \mu \mathrm{W}$ in listening mode. The average power con1139 sumption of CargoNet is $23.7 \mu \mathrm{W}$ when the node is active and 1140 receiving the data packet via the main transceiver. At maxi1141 mum sensitivity of $-65 \mathrm{dBm}$, the WuRx is able to detect an 1142 OOK modulated WuS up to a distance of $8 \mathrm{~m}$.

1143 Ansari et al. [38] presented a radio triggered wake-up cir1144 cuit attached to a TelosB node and exploited its main MSP430 1145 MCU for address decoding. The WuTx uses an additional out1146 of-band $868 \mathrm{MHz}$ CC1000 transceiver for generating WuS 1147 using Pulse Interval Encoding (PIE) scheme and a frequency 1148 amplifier for communication range extension. The main build1149 ings blocks include an impedance matching network, a voltage 1150 multiplier and a digital comparator interfaced to the main 1151 MCU. The matching network is constructed using discrete 1152 components such as capacitors and inductors while the 5-stage 1153 voltage multiplier uses RF Schottky diodes. The MCU tracks 1154 the low-to-high transitions and the time intervals between the 1155 PIE signal to successfully decode the data. In case the wake1156 up packet is not addressed to the node, it switches back to the 1157 sleep mode. Otherwise, the node triggers its main CC2420 1158 transceiver for data exchange. The WuRx in listening mode 1159 consumes only $2.628 \mu \mathrm{W}$ and the micro-controller consumes $11601020 \mu \mathrm{W}$ when it switches from sleep to active mode for 1161 address decoding. Empirical measurements using simulation 1162 shows that the proposed WuRx has an operating range of $10 \mathrm{~m}$ 1163 for the $500 \mu \mathrm{W}$ transmission power.

1164 Le-Huy and Roy [32] also simulated a semi-active WuRx 1165 that uses correlator as a decoder. This work has become one 1166 of the reference designs for several newer proposals, since 1167 authors have outlined the complete steps from signal detection 1168 to address comparison. The proposed architecture consists of 1169 a shared antenna between the $\mathrm{WuRx}$ and the main transceiver, 1170 impedance matching network and zero-bias Schottky diode 1171 based envelope detector. It is followed by an address decoder 1172 circuit that has three subsystems: the amplifier stage, the PWM 1173 demodulator and the correlator circuit consisting of shift reg1174 ister and a logic comparator. The power consumption of the 1175 proposed architecture is $19 \mu \mathrm{W}$ at a data rate of 50kbps with 1176 receiver sensitivity of $-53 \mathrm{dBm}$. Using Pulse Width Modulation 1177 scheme, the receiver exhibits a maximum range of $5 \mathrm{~m}$ for $11782.4 \mathrm{GHz}$ band.

1179 Ammar et al. [103] also proposed a semi-active $868 \mathrm{MHz}$ 1180 WuRx that uses Flip Flops for address decoding and dissipates 1181 only $13.41 \mu \mathrm{W}$. However, this design is only evaluated using 1182 simulations. Other simulated designs based on semi-active 1183 WuRxs can be found in [105], [109], [110], and [118].

1184 Gamm et al. [36] proposed the first in-band sub-Carrier 1185 modulation WuRx system based on AS3932 (Fig. 11). In the 1186 wake-up mode the WuS is directed to the AS3932 WuRx 1187 for envelope and address decoding after impedance matching 1188 and demodulation of OOK signal. First, AS3932 extracts the $1189125 \mathrm{KHz}$ signal from the $868 \mathrm{MHz} \mathrm{WuS}$ and then the original 1190 data is decoded for address comparison. Once the address is

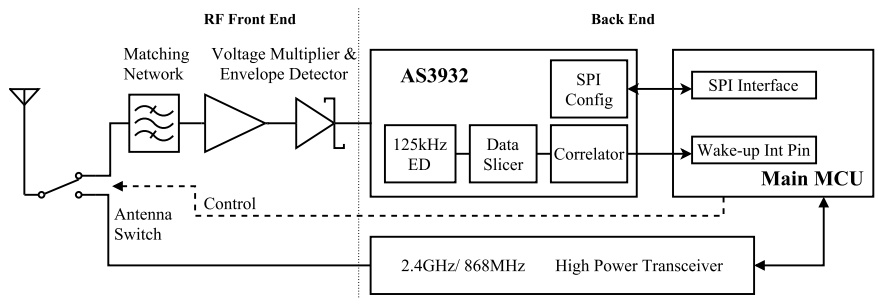

Fig. 11. Wake-up receiver prototype utilizing Austria Micro Systems AS393x WuRx [36].

matched, the main node is triggered. Afterwards, an antenna 1191 switch is utilized to bypass the WuRx and the data exchange 1192 takes place using the main CC1101 transceiver. The main radio 1193 is also utilized as a WuTx to generate the WuS, thus the first 1194 complete WuR transceiver. The WuRx circuitry is supplied 1195 with $3 \mathrm{~V}$ battery and has an active power consumption of 1196 $7.8 \mu \mathrm{W}$ while the total node consumption is $44 \mathrm{~mW}$. For an 1197 output power of $11 \mathrm{dBm}$ at the WuTx, the maximum wake-up 1198 distance was $45 \mathrm{~m}$ at a data rate of $250 \mathrm{kbps}$ and sensitivity 1199 level of $-52 \mathrm{dBm}$. The design by Gamm et al. [36] has become 1200 the starting point for other AS393X based WuR systems such 1201 as the ones presented in [50], [53], and [56]. 1202

The most energy efficient semi-active WuRx proposed to- 1203 date is presented by Roberts and Wentzloff [111]. The energy 1204 is harvested from the RF signal and then the received voltage is 1205 boosted using resonant tank before supplied to the active part 1206 of the circuit. This $915 \mathrm{MHz}$ band WuRx achieved a commu- 1207 nication range of $1.2 \mathrm{~m}$ at transmission power of $0 \mathrm{dBm}$. The 1208 whole CMOS based WuRx provides a data rate of $100 \mathrm{kbps} 1209$ using OOK modulation while consuming only $98 \mathrm{nW}$ in active 1210 state. However, the WuRx does not support node addressing 1211 as per the implementation.

Yet another ultra-low power WuRx intended for WBAN is 1213 presented in [104]. The proposed design uses Gaussian On- 1214 Off Keying (GOOK) and Pulse Width modulation (PWM) for 1215 decoding and encoding the preamble signal, respectively. This 1216 receiver has higher power consumption of $2.67 \mu \mathrm{W}$ than that 1217 proposed by Roberts and Wentzloff [111] in listening mode, 1218 but achieves a longer communication range of $10 \mathrm{~m}$ for WuTx 1219 output power of $10 \mathrm{dBm}$. The WuRx also operates in a sub- 1220 $\mathrm{GHz}$ frequency band (433 MHz) and has receiver sensitivity 1221 of $-51 \mathrm{dBm}$. The address decoding is handled by the MCU 1222 and the authors have not provided any details of its related ${ }_{1223}$ power consumption.

To increase the flexibility of WuR, multi-band WuRs have 1225 also been exploited to allow interoperability between differ- 1226 ent frequencies used in WSNs. Huang et al. [115] propose a 1227 radio-triggered WuRx able to operate selectively at $915 \mathrm{MHz} 1228$ and $2.4 \mathrm{GHz}$ band. After input matching, an envelope detec- 1229 tor suppresses the fundamental tone to the required frequency 1230 followed by a baseband amplifier for filtering and amplifying 1231 the WuS. This WuRx consumes $51 \mu \mathrm{W}$ for $100 \mathrm{kbps}$ OOK 1232 modulation featuring receiver sensitivity of $-75 \mathrm{dBm}$ in the ${ }_{123}$ $915 \mathrm{MHz}$ band and $-64 \mathrm{dBm}$ in $2.4 \mathrm{GHz}$ band, respectively. ${ }_{1234}$

Oh et al. [116] presented a tri-band $116 \mathrm{nW}$ WuRx 1235 with 31-bit Correlator with interference rejection capabilities. 1236 
1237 The WuRx front end operates in the $402 \mathrm{MHz}$ MICS band 1238 and the $915 \mathrm{MHz}$ and $2.4 \mathrm{GHz}$ ISM band with sensitivities 1239 of $-45.5 \mathrm{dBm},-43.4 \mathrm{dBm}$ and $-43.2 \mathrm{dBm}$, respectively. The 1240 chip consists of an input matching network for filtering and 1241 boosting the incoming $\mathrm{WuS}$ and a 30-stage passive rectifier 1242 for down-converting the RF signal to baseband, which is then 1243 sensed by a comparator. Finally, a bank of 124 correlators is 1244 implemented to compare the wake-up sequences with a pro1245 grammable wake-up code. The wake-up interrupt is generated 1246 only when a correlation value exceeds a user-programmable 1247 threshold.

1248 Recently, another dual-band WuRx that operates in ${ }_{1249} 868 \mathrm{MHz}$ and $2.4 \mathrm{GHz}$ band has been proposed in [105]. 1250 The WuRx front end consists of a dual-band antenna and 1251 matching network with a passive envelope detector. The back1252 end consists of an interrupt/data generator and an ultra-low 1253 power micro-controller for address decoding and generating 1254 interrupt to the sensor node. The receiver is tuned to use ${ }_{1255} \mathrm{OOK}$ modulation for $\mathrm{WuS}$ with sensitivity of $-53.4 \mathrm{dBm}$ 1256 and $-45.2 \mathrm{dBm}$ at $868 \mathrm{MHz}$ and $2.45 \mathrm{GHz}$, respectively. 1257 Simulation results demonstrate that the proposed solution con1258 sumes $1.276 \mu \mathrm{W}$ while listening the channel and this power 1259 consumption increases to $70.6 \mu \mathrm{W}$ when the MCU is decoding 1260 the address with supply voltage of $1.8 \mathrm{~V}$.

\section{${ }_{1261}$ D. Non-RF Based WuRs}

1262 While RF based WuRs have been most widely researched, 1263 some authors have proposed an unconventional method to 1264 communicate with the WuRx by exploiting different transmit1265 ting mediums like optical or ultrasonic signals. For this reason 1266 it is quite inappropriate to call such devices WuR, but still 1267 some solutions are interesting and expose characteristics that 1268 are comparable with RF based WuRs discussed so far. In fact 1269 the communication range that could be achieved with these 1270 type of wake-up transceivers are similar to typical RF based ${ }_{1271} \mathrm{WuRs}$ while also exhibiting similar power demands. The two 1272 main drawbacks are that some of these devices require direc1273 tionality and/or line-of-sight (LOS) communication between 1274 transmitter and receiver, making them inappropriate for some 1275 applications. The complete list of all the WuRs in this category 1276 is presented in Table IX.

1277 Hakkinen and Vanhala [120] proposed one of the earli1278 est designs where infrared is utilized to transmit WuS. The ${ }_{1279}$ WuTx is basically an IR LED that is switched on and off by 1280 the micro-controller. On the WuRx side, a photo-detector is 1281 used for receiving the signal and a transimpedence amplifier 1282 converts this signal into voltage to generate an interrupt. It 1283 achieves operational range of up to $30 \mathrm{~m}$ with an IR remote 1284 controller by matching its carrier frequency with the WuRx. 1285 The prototype consumes $12 \mu \mathrm{W}$ when listening for the WuS 1286 at a supply of $3 \mathrm{~V}$. Unfortunately, the wake-up circuit is very 1287 sensitive to external light and is vulnerable to noise while 1288 requiring direct LOS between nodes.

1289 The proposal by Mathews et al. [48] utilizes Free Space 1290 Optical (FSO) as a secondary wake-up channel. The power 1291 consumption of the proposed FSO WuRx is $317 \mu \mathrm{W}$ in lis1292 tening mode and attains a LOS range of $15 \mathrm{~m}$ at a transmission power of $16.5 \mathrm{~mW}$. Due to low gain bandwidth of the oper- 1293 ational amplifiers, the system suffers from low data rate of 1294 2 kbps. Optical based designs implicitly feature node address- 1295 ing through directional communication, however, it is not clear 1296 how this design would perform when the nodes are not per- 1297 fectly aligned and how to communicate with multiple nodes, 1298 if required.

1299

Another optical based WuRx is presented in [49] called 1300 Free-space Low-Power optical Wake-up and has an ultra low 1301 power of only $695 \mathrm{pW}$ in standby mode and $12.2 \mathrm{nW}$ in 1302 active mode. The WuR supports three different light sources 1303 for extending communication range. Using 0.5 W LED the 1304 wake-up range is $0.2 \mathrm{~m}, 6 \mathrm{~m}$ with $3 \mathrm{~W}$ LED with focus and 1305 extends to $50 \mathrm{~m}$ when a $3 \mathrm{~mW}$ green laser is utilized as WuTx. 1306 In contrast to [48], FLOW features a 16-bit node addressing 1307 capability. However, similar to [48], the WuR system requires 1308 direct LOS for transmitting WuS and supports very low bit 1309 rate of 91 bps. Moreover, to achieve long range communica- 1310 tion, proper physical alignment between the optical WuTx and 1311 WuRx is also required.

Sánchez et al. [47] have presented an asynchronous 1313 acoustic-triggered wake-up modem for underwater sensor 1314 networks. Using this technique, the WuRx is programmed to 1315 react to acoustic signals at a certain frequency, reactivating 1316 the node if needed. The WuRx consumption is $10 \mu \mathrm{W}$ in lis- 1317 tening mode. The authors have also integrated AS3933 for 1318 16-bit node address recognition. With a transmission power 1319 of $108 \mathrm{~mW}$, an underwater communication range of $240 \mathrm{~m} 1320$ has been achieved.

1321

An ultrasonic WuRx working at $40.6 \mathrm{kHz}$ is proposed 1322 in [44]. It uses piezoelectric transducer that converts the 1323 mechanical energy into electrical energy for generating wake- 1324 up interrupts. The design is based on heterodyne architecture 1325 and the overall receiver power consumption is $4.8 \mu \mathrm{W}$ in lis- 1326 tening mode. When exciting the transmitter with an electrical 1327 signal power of $16 \mu \mathrm{W}$, it achieved an operational range of 1328 $8.6 \mathrm{~m}$. However, the WuRx has very low bit rate of $250 \mathrm{bps} .1329$ Another prototype using ultrasonic signals is presented by 1330 Lattanzi et al. [45]. Unlike [44], this design supports out- 1331 of-band addressing scheme for selective awakening. It uses 1332 off-the-shelf components and requires $1.748 \mu \mathrm{W}$ in listening 1333 state and around $14 \mu \mathrm{W}$ when active. This design is suitable 1334 for ranging applications that require distance up to $10 \mathrm{~m}$. The 1335 WuTx takes $0.5 \mathrm{~s}$ to transmit an 8-bit address and requires 1336 $75 \mu \mathrm{W}$ of power at bit rate of $16 \mathrm{bps}$.

The design by Hoflinger et al. [46] presents an acous- 1338 tic WuRx operating at $18 \mathrm{kHz}$ for controlling devices and 1339 appliances at home. The audio signal is sent using a smart- 1340 phone speaker and a micro-electromechanical system (MEMS) ${ }_{341}$ microphone is used to detect the audio signal on the WuRx. 1342 The microphone transducer converts this acoustic signal into 1343 an electrical signal, which is then fed into AS3933 WuRx IC 1344 that detects a valid frequency of $18 \mathrm{kHz}$ and triggers the micro- 1345 controller. A wake-up range of $7.5 \mathrm{~m}$ was achieved using this 1346 setup. The WuRx consumes $56 \mu \mathrm{W}$ in listening mode while ${ }_{334}$ the consumption hikes to $440 \mu \mathrm{W}$ in active state when receiv- 1348 ing the signal using PWM modulation. This design was further 1349 improved in [121], which operates at $20 \mathrm{kHz}$ audio signals and 1350 
1351 features node addressing. To reduce the power consumption 1352 compared to [46], the power amplifier and the microphone are 1353 duty cycled using the micro-controller. Using this technique, 1354 the proposed design attains a power consumption of $45 \mu \mathrm{W}$ 1355 in listening mode and $420 \mu \mathrm{W}$ in active mode. An average 1356 wake-up range of $10 \mathrm{~m}$ using smart-phone as a sender was 1357 achieved.

1358 Recently, Carrascal et al. [122] have developed a visible 1359 light communication (VLC) based WuR system. This system ${ }_{1360}$ uses an off-the-shelf indoor solar panel as a receptor and ${ }_{1361}$ energy harvester to power the WuRx. The WuRx is also cou1362 pled with AS3933. At the transmitter side, a $10 \mathrm{~W}$ LED is 1363 modulated using OOK at a frequency of $21 \mathrm{kHz}$ to transmit ${ }_{1364} \mathrm{WuS}$. In an indoor environment, with short bit duration the 1365 prototype achieved $7 \mathrm{~m}$ range while with longer bit duration 1366 maximum achievable range was $14 \mathrm{~m}$. This VLC based WuR 1367 consumes $19.2 \mu \mathrm{W}$ in listening mode and $\sim 95 \mu \mathrm{W}$ when 1368 receiving and decoding the $\mathrm{WuS}$. The transmission power 1369 required to achieve the above range was $87.9 \mathrm{~mW}$ at a data 1370 rate of $1.12 \mathrm{kbps}$. The proposed system is suitable for indoor 1371 applications only and allows to harvest energy from the indoor 1372 lights for energy-autonomous operation of the WuRx.

\section{E. Summary}

1374 In Section III, we considered different physical layer char1375 acteristics of wake-up receivers, each designed and tested 1376 in separate ways. We next discuss some of the advantages, 1377 disadvantages, and features for each category.

1378 From the application point of view, RFID-based WuR 1379 systems are suited for mid-range applications such as health 1380 monitoring, inventory monitoring, or environmental applica1381 tions [102]. Nonetheless, the maximum communication range 1382 achieved so far has been $30 \mathrm{~m}$ using an active RFID tag [90]. ${ }_{1383}$ As active RFID tags are costly and require more power, such ${ }_{1384} \mathrm{WuR}$ designs may not be suitable for applications that require 1385 extended lifetime with minimum maintenance. Moreover, the 1386 communication range of RFID devices are related to antennae 1387 size: the bigger the antenna the more power can be transmit1388 ted thus the longer the range. For WuR based applications 1389 that demand small form factor, this could be a hindrance and 1390 may force designers to opt for other technologies such as 1391 system-on-chip, which may be suitable for a wide range of 1392 applications. In addition, for passive RFIDs and EH-WuRxs 1393 not all energy is absorbed by the receiving end resulting 1394 in a phenomenon known as backscattering. Thus, WuS are 1395 transmitted at high power and usually take a few seconds 1396 to accumulate and recharge the capacitors for powering up 1397 the circuits. This, in turn, affects the wake-up range and the 1398 latency of the system as a whole.

1399 Most active WuRs use CMOS technology and a heterodyne 1400 approach. While these heterodyne-based WuRs offer superior 1401 sensitivity and data rate, most lack node addressing capabili1402 ties and information on their operational range. This category ${ }_{1403}$ of $\mathrm{WuR}$ also features the highest power consumption of up to 1404 a few milliwatts [87], [89] as the heterodyne approach requires 1405 some active components such as IF-amplifiers and mixers. It 1406 has also been noticed that some of these designs operate in 1407 lower non-ISM bands such as $45 \mathrm{MHz}$ [71] or $1.9 \mathrm{GHz}$ [60] making them inadequate for medical applications. By con- 1408 trast, lower operation frequency may enable the design of 1409 transceivers that consume less power than transceivers in 1410 higher frequencies. Moreover, it enhances security compared 1411 to traditional wireless technologies for WBAN by making the 1412 radio signal more difficult to eavesdrop.

1413

It has also been observed that the use of a secondary MCU 1414 for address decoding allows faster prototyping at the receiver 1415 back-end. On the other hand, the introduction of this extra 1416 hardware adds to the overall power overhead and may not be 1417 applicable for applications that have strict power requirements. 1418 However, due to advancements in miniaturization, the power 1419 consumption of these MCUs has drastically reduced over the 1420 years making it possible to integrate with WuRx while still ${ }_{1421}$ achieving power consumption below $10 \mu \mathrm{W}$.

1422

The choice of modulation scheme also affects the overall ${ }_{1423}$ WuRx performance. If a complex modulation technique like ${ }_{1424}$ FSK is utilized, this demands complex circuitry at the RF 1425 front-end such as the use of active demodulators, mixers, and 1426 amplifiers that require extra power. Therefore, simple modula- 1427 tion techniques such as OOK and ASK presents an opportunity 1428 to simplify the WuRx circuitry and to achieve low power con- 1429 sumption. Most of the WuRxs reported are compatible with 1430 only one of these two modulations. As a consequence, the 1431 WuRx architecture implemented in wireless nodes can limit 1432 the interoperability with other transmitters.

\section{STATISTICAL ANALYSis}

1434

Different components of the WuR design impact its final 1435 performance and add to its overall power consumption. In 1436 this section, we compare different RF based WuR prototypes 1437 designed and tested in terms of power consumption, sensitivity, 1438 data rate, communication range and the modulation scheme 1439 used, regardless of their specific technology. The numbers 1440 presented in this section are the actual numbers reported by 1441 the authors of each article. This statistical comparison will 1442 then be used as a guideline to recommend which prototypes 1443 are suitable and meet the requirements of various applications 1444 outlined in Section VIII.

\section{A. Modulation Schemes}

The main goal of incorporating WuR with typical sensor 1447 node is to reduce power consumption. In order to achieve this, 1448 the WuR design should be of low power, hence, the modulation 1449 complexity should be kept low as well. The higher the modu- 1450 lation complexity, the more stringent requirements for receiver 1451 and transmitter in terms of circuit complexity and power. 1452

When comparing this with the state-of-the-art low power 1453 WuR summarized in Table VIII, it can be noted that most 1454 designs use either envelope detector based On-Off key- 1455 ing (OOK) or non-coherent Frequency-Shift-Keying (FSK). To 1456 curb energy consumption by simplifying overall implementa- 1457 tion, the designers of the WuR generally favor architectures 1458 utilizing OOK modulation schemes. For instance, a sim- 1459 ple envelope detector using few diodes and capacitors can 1460 be used for signal detection [40], [54], [104]. It is evident ${ }_{1461}$ from Table VIII that most of the concepts that have power 1462 consumption below $10 \mu \mathrm{W}$ are using OOK modulation. 


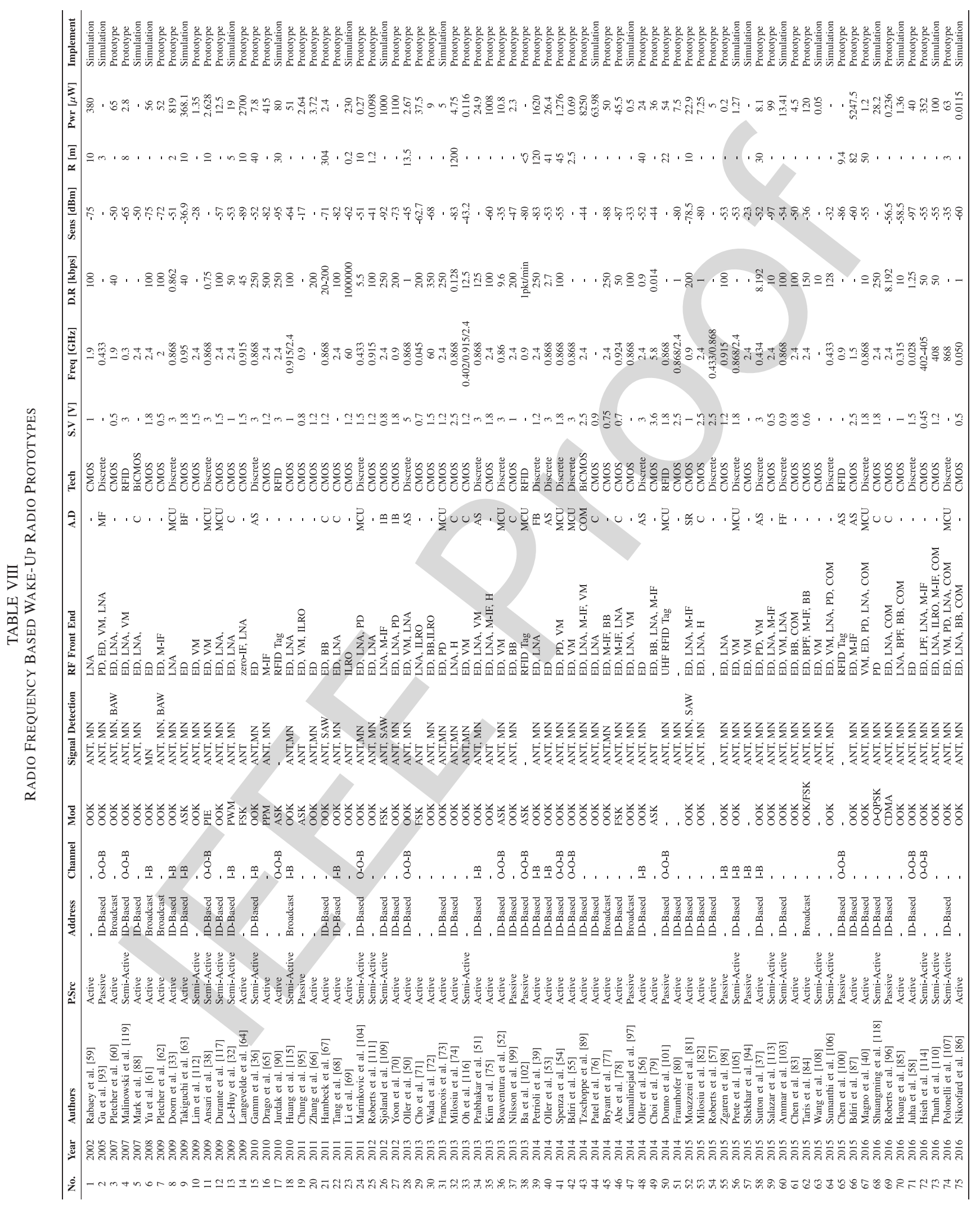




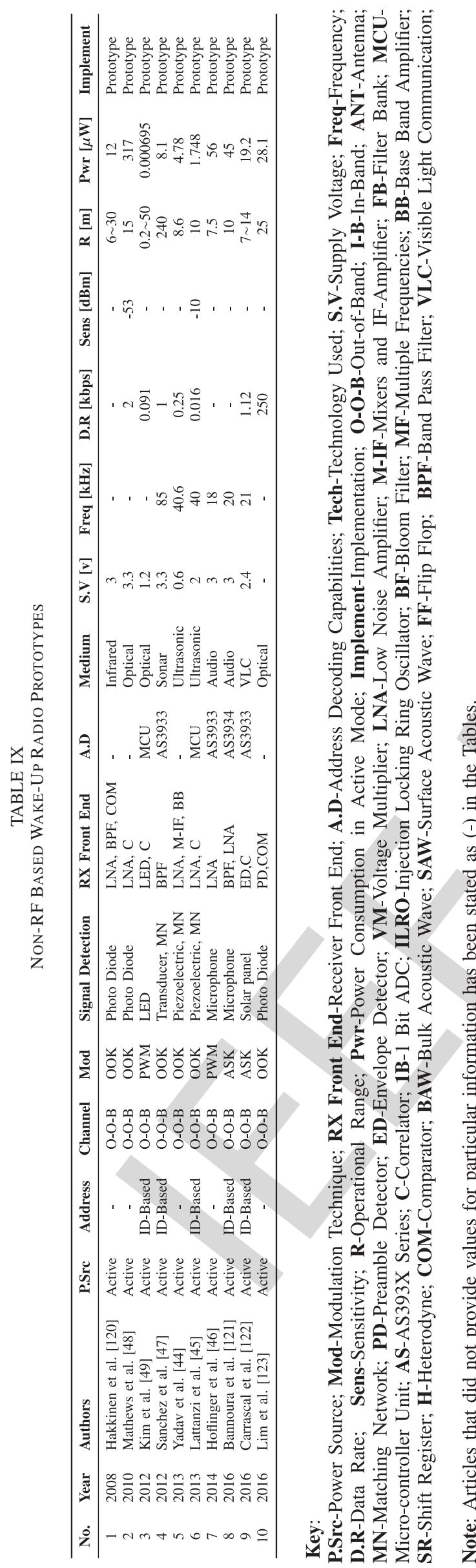

In contrast, the nonlinear nature of envelope detectors make 1464 the OOK receivers more susceptible to interference contribut- 1465 ing to higher packet error rate and need for retransmission. One 1466 can argue that retransmission is expensive in terms of power, ${ }_{1467}$ but the burden of this is shifted from high power radio to ultra- 1468 low power WuR. The advantage of FSK over OOK is that it ${ }_{1469}$ is more resilient to fading and interference. Therefore, in view 1470 of low power WuRx design, either OOK or FSK modulation 1471 scheme should be considered.

There are five reported design concepts that differ from 1473 above. The concept presented by Le-Huy and Roy [32] 1474 uses Pulse-width modulation (PWM) technique since it only 1475 requires an integrator with a reset option without increasing 1476 the complexity of the receiver architecture. Another benefit of 1477 using PWM is that it presents the possibility to control the duty 1478 cycle of the transceiver. Shuangming et al. [118] use the Offset 1479 quadrature phase-shift keying (O-QPSK) to design an ultra low 1480 power System-on-Chip (SoC) based baseband processor with ${ }_{1481}$ wake-up identification receiver consuming only $28.2 \mu \mathrm{W}$. The 1482 concept by Ansari et al. [38] use multi-stage approach for ${ }_{1483}$ WuSing where CC1000 radio chip is used to perform OOK 1484 by turning on and off it's power amplifier. Then the digital 1485 data is encoded using Pulse Interval Encoding (PIE) with dif- 1486 ferent time intervals $T$. In order to successfully decode this ${ }_{1487}$ data sequence, authors utilize MSP430 series micro-controller. 1488 A broadband-IF super heterodyne proposal for a crystal-less 1489 2.4 GHz WuRx is presented by Drago et al. [65]. The WuS 1490 is modulated by means of Pulse Position Modulation (PPM). 1491 In order to reduce the power consumption of their design, 1492 both the signal front-end and the oscillator are duty-cycled ${ }_{1493}$ at the pulse level. The WuRx achieves $-82 \mathrm{dBm}$ sensitivity 1494 and requires up to $415 \mu \mathrm{W}$. Recently, Roberts et al. [96] 1495 have proposed a Bluetooth Low Energy (BLE) WuRx with 1496 energy harvesting capability. They have utilized Code divi- 1497 sion multiple access (CDMA) modulation scheme referred to 1498 as Back-channel for encoding and decoding the WuS. Upon 1499 signal detection, the information is fed into a baseband proces- 1500 sor that correlates the energy levels with a time-based template 1501 that matches the sequence of BLE advertising packets to deter- 1502 mine the presence of a wake-up message. This CMOS based ${ }_{1503}$ design was able to achieve sensitivity of $-56.5 \mathrm{dBm}$ while ${ }_{1504}$ consuming only $236 \mathrm{nW}$.

$$
1505
$$

\section{B. Sensitivity vs. Power Consumption}

1506

Fig. 12 shows the comparison between the WuR's power ${ }_{1507}$ versus sensitivity. It should be noted that these are all custom 1508 ultra-low power radios, including radios of different architec- 1509 ture, different data rate, different operating frequencies; none 1510 of which is separated in this plot.

1511

Generally, the power consumption of the WuR is related to 1512 its sensitivity. With power consumption, in $\mu \mathrm{W}$, on the y-axis 1513 and the sensitivity, in $\mathrm{dBm}$, on the $\mathrm{x}$-axis, two distinct trends 1514 can be observed. First, when looking at sensitivity higher than 1515 $-40 \mathrm{dBm}$ (to the left on the $\mathrm{x}$-axis) it can be seen that there is 1516 no direct correlation between the changing sensitivity to the 1517 power of the receiver. However, there is a floor around $2 \mu \mathrm{W} 1518$ suggesting that there is a minimum power requirement for 1519 


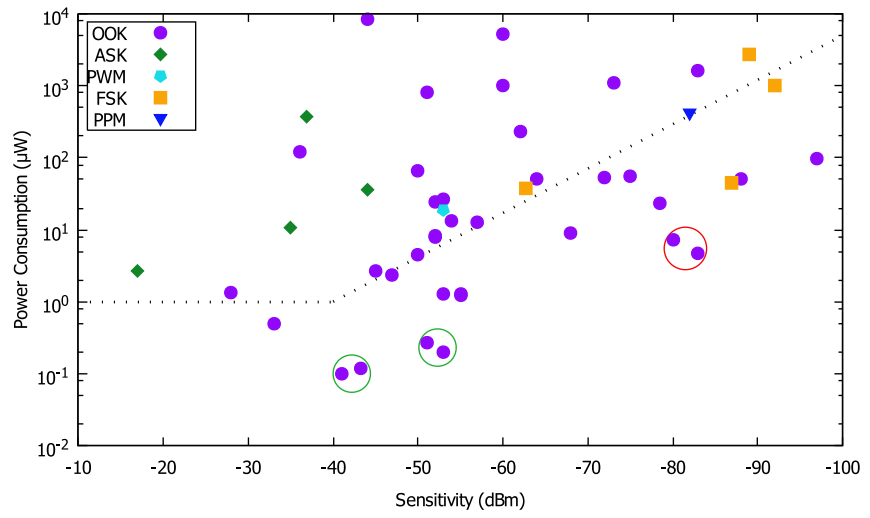

Fig. 12. Sensitivity of low power RF based wake-up receivers vs. Power consumption w.r.t different signal modulation techniques.

1520 the radio regardless of sensitivity. With increasing sensitivity 1521 from $-40 \mathrm{dBm}$ (to the right on the $\mathrm{x}$-axis) there is a liner trend 1522 indicating a correlation between sensitivity and power. It can 1523 be seen empirically through slope-fitting that a $20 \mathrm{dBm}$ change 1524 in sensitivity results in an approximately $10 \times$ change in power 1525 consumption. The designs below this slope are regarded as 1526 energy efficient as most of them exhibit high sensitivity at 1527 low energy cost.

1528 Moreover, as can be seen in Fig. 12, the lowest power con1529 sumption that has been achieved so far has been $98 \mathrm{nW}$ [111], 1530 but not without trading-off the sensitivity $(-41 \mathrm{dBm})$. This 1531 design was able to achieve a communication range of 1532 only $1.2 \mathrm{~m}$. Out of 75 prototypes that we have sur1533 veyed for RF based WuR for those that power consumption 1534 and sensitivity values were provided, only 23 prototypes 1535 were able to achieve power consumption below $10 \mu \mathrm{W}$, 1536 where [86], [111], and [116] reached an outstanding power 1537 consumption around $100 \mathrm{nW}$.

${ }_{1538}$ Regarding the requirements for different applica1539 tions in Table XII, it can be seen that for short-range 1540 communication such as WBAN, five WuR proto1541 types [96], [98], [104], [111], [116] (marked with green 1542 circles) fulfill the power consumption and sensitivity require1543 ments. All these prototypes have power consumption below ${ }_{1544} 0.27 \mu \mathrm{W}$ with sensitivity ranging between $-40 \mathrm{dBm}$ to $1545-56 \mathrm{dBm}$. For mid-range communication (e.g., smart city and 1546 metering), only [74], [82] (marked with a red circle) fullfill 1547 all these requirements at the same time. Power and sensitivity 1548 of these prototypes are $4.75 \mu \mathrm{W}$ and $7.25 \mu \mathrm{W}$, and $-83 \mathrm{dBm}$ 1549 and $-80 \mathrm{dBm}$, respectively.

1550 For ultra-low power WuR, the knowledge from Fig. 12 is 1551 useful for understanding key design trade-offs. For example, 1552 most designers [64], [87], [89] try to push the sensitivity as 1553 low as possible to achieve better communication range, but 1554 this may lead to power-costly design.

1555 In terms of modulation technique, most of these designs 1556 utilize OOK modulation. OOK based prototypes have been 1557 able to reach the two extreme ends of the power levels, one 1558 being the most energy efficient [111] while the other design 1559 is not [87]. There are two designs, one based on CDMA [96] 1560 and the other using FSK modulation [78] that have also been 1561 able to achieve an excellent receiver sensitivity of $-56.5 \mathrm{dBm}$

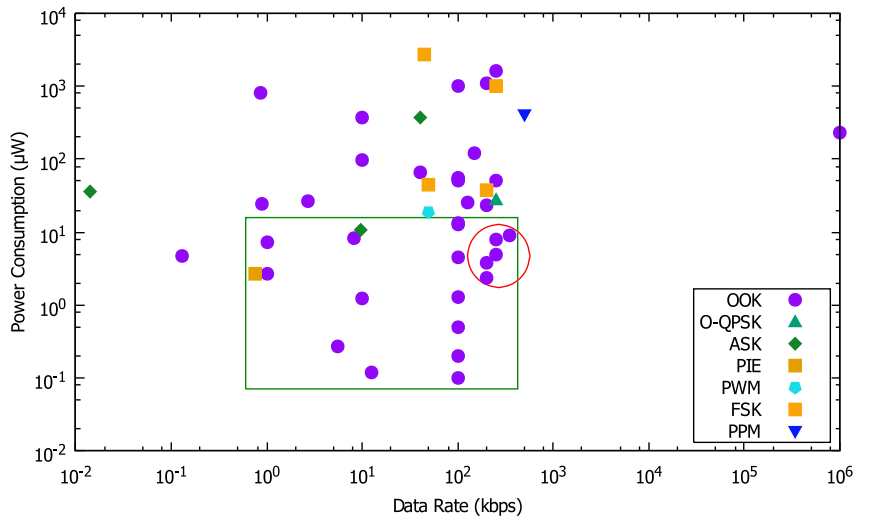

Fig. 13. Data Rate of low power RF based wake-up receivers vs. their Power consumption w.r.t different signal modulation techniques.

and $-87 \mathrm{dBm}$, respectively with very low power requirements. 1562 Both of these prototypes are fabricated using 65nm CMOS 1563 process and use correlators for address decoding.

1564

\section{Data Rate vs. Power Consumption}

1565

Fig. 13 shows the data rate of WuRxs with respect to their 1566 power consumption and signal modulation techniques. Since, 1567 power is inversely proportional to data rate, it is generally pos- 1568 sible to increase the data rate with little power overhead [124], 1569 however, communication distance will be short. For example, 1570 it does not cost much in terms of power to increase the mod- 1571 ulation rate from $1 \mathrm{kbps}$ [50] to $100 \mathrm{kbps}$ [83] in an OOK 1572 receiver.

1573

As can be seen, there are fourteen designs that have been 1574 able to reach a data rate above $200 \mathrm{kbps}$. Out of these, 1575 five [36], [66], [72], [73], [99] have a power consumption 1576 below $10 \mu \mathrm{W}$.

1577

From the application perspective, there are few 1578 designs [36], [66], [72], [73], [99] (circled in red) that 1579 offer high data rate at the same time consuming low power 1580 making them suitable for WBAN application scenarios for 1581 replacing the high data radio with WuR. Thanks to its high 1582 data rate and low power consumption, these WuR utilized 1583 as main data radio can have an advantage over duty cycled 1584 transceiver in terms of reducing the overall communica- 1585 tion delay. One of the prototypes in the millimeter-wave 1586 band operating at $60 \mathrm{GHz}$ based on OOK modulation has 1587 been designed to achieve very high data rate of up to 1588 1 Gbps [69], however, it may not be applicable for WBAN 1589 due to its high power consumption of $230 \mu \mathrm{W}$. However, 1590 this makes it suitable for wireless personal area network 1591 applications that demand short-range of up to $0.2 \mathrm{~m}$ with high 1592 data rate.

1593

For mid-range applications that require moderate data rates 1594 with low power consumption, there are few prototypes (green 1595 rectangle) that may be suitable for these scenarios. All these 1596 prototypes have data rate between $0.75 \mathrm{kbps}$ to $500 \mathrm{kbps}$, and 1597 power consumption below $12.5 \mu \mathrm{W}$.

1598

\section{Range and Frequency Usage}

1599

So far we have only looked at the modulation technique, 1600 receiver sensitivity, and data rate. Another factor that impacts 1601 


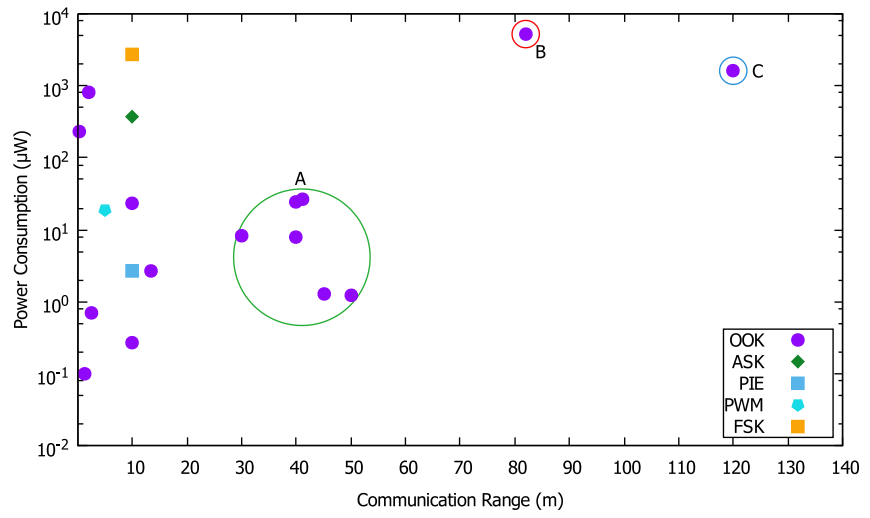

Fig. 14. Communication Range of RF based wake-up receivers vs. their Power consumption w.r.t different signal modulation techniques.

1602 the power consumption of wake up radios is the carrier 1603 frequency. The choice of the carrier frequency is an impor1604 tant parameter for the wake-up transceiver. Fig. 15 shows 1605 the main frequency bands that have been utilized by most of 1606 the WuR prototypes together with the min, max and average 1607 power consumption. One of the trends that can be observed is 1608 that the average power consumption of transceivers increases 1609 from sub-GHz band to $2.4 \mathrm{GHz}$. This is due to the fact that 1610 transceiver circuits running at higher frequencies require more 1611 current to achieve the same performance as lower frequencies. 1612 From this survey and referring to Table VIII, it can be seen 1613 that 25 of the prototypes are based on $2.4 \mathrm{GHz}$ while 32 1614 of them are between $433 \mathrm{MHz}$ and $915 \mathrm{MHz}$. One of the 1615 designs that have achieved an outstanding power consump1616 tion of $0.0115 \mu \mathrm{W}$ operates in $50 \mathrm{MHz}$ [86]. The design is 1617 based on CMOS technology and features a data rate of $1 \mathrm{kbps}$ 1618 with receiver sensitivity of $-60 \mathrm{dBm}$. Due to its semi-active 1619 design and OOK modulation, this particular prototype man1620 aged to surpass state-of-the-art wake-up radios in terms of 1621 sensitivity and power trade-off. However, it has only been 1622 tested via simulations. Nevertheless, most of the designers 1623 have opted to shift from high frequency to sub- $\mathrm{GHz}$ as an 1624 operating frequency for wake-up receivers. One of the reasons 1625 is that at higher frequencies the attenuation rate also increases, 1626 i.e., the $2.4 \mathrm{GHz}$ signal weakens faster than a sub-GHz signal. 1627 According to the Friis equation, the path loss at $2.4 \mathrm{GHz}$ is ${ }_{1628} 8.5 \mathrm{~dB}$ higher than at $900 \mathrm{MHz}$ translating into 2.67 times 1629 longer range for $900 \mathrm{MHz}$ transceivers.

${ }_{1630}$ Besides the need for higher power for the same link budget, ${ }_{1631} 2.4 \mathrm{GHz}$ band is more prone to interference due to spectrum 1632 crunch and devices such as Wi-Fi and Bluetooth operating in 1633 the same band. Sub-GHz ISM bands are mostly used for pro${ }_{1634}$ prietary low-duty-cycle links and are not as likely to interfere 1635 with each other. The quieter spectrum means easier trans1636 missions and fewer retries, which is more efficient and saves ${ }_{1637}$ battery power for wake-up radio based systems.

${ }_{1638}$ Furthermore, Fig. 14 shows the maximum achievable com1639 munication range reported for different WuR prototypes 1640 in terms of their power consumption. It should be noted 1641 that we do not take into account prototypes that did not 1642 report explicitly the communication range of the WuR.

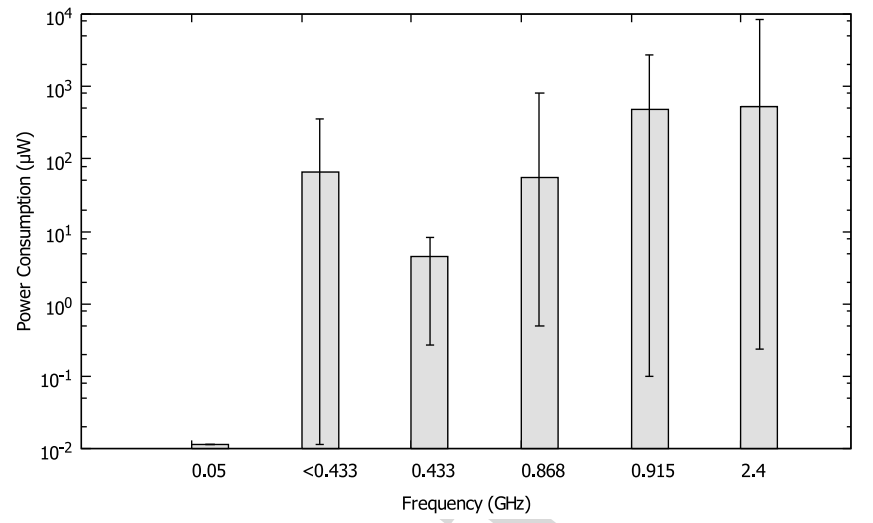

Fig. 15. Frequency selection vs. Power consumption.

From the application point of view, WuR prototypes with 1643 communication range between $30 \mathrm{~m}$ to $50 \mathrm{~m}$ (labeled as 1644 cluster A) [36], [37], [40], [53], [54] satisfy the requirements 1645 for mid-range applications. For the WBAN case WuR con- 1646 cepts [36], [66], [72], [73], [99] fulfill the sensitivity, data 1647 rate and power requirements, if used as a full data radio. 1648 However, if utilized just as a secondary radio for triggering 1649 the main node's transceiver, WuR with power consumption 1650 below $10 \mu \mathrm{W}$ should be considered.

E. Summary

1652

The main characteristics of all ultra-low power WuR are 1653 sensitivity, data rate, frequency, and power consumption. 1654 However, the technology used to design WuR prototypes 1655 vary from simple energy detection using discrete components 1656 to envelope detection using CMOS, influencing its overall 1657 performance. Therefore, for different application requirements 1658 the best prototype has to be selected carefully. While some 1659 provide high data rate, others are better for high sensitivity or 1660 very low power consumption.

1661

It has been observed that to achieve ultra-low-power con- 1662 sumption while maintaining robust operation involves difficult 1663 trade-offs between range, data rate, sensitivity, and energy 1664 efficiency that must be overcome through a combination of 1665 innovative circuit design, novel architectures, and system-level 1666 considerations. This section has provided some benchmarking 1667 data to help identify what architectures and WuR prototypes 1668 might make the most sense given system-level specifications. 1669 While optimal implementations depend strongly on the given 1670 application, in general the most energy efficient WuR employ 1671 low-complexity modulation schemes (e.g., OOK).

\section{Medium Access Control}

1673

Major work on WuR technology has been focused on 1674 improving hardware components to achieve better power con- 1675 sumption and physical layer communication characteristics. 1676 Nevertheless, to fully exploit the technology, it must be cou- 1677 pled with communication protocols, rounding out the system 1678 design. We divide our discussion in two parts, first focusing 1679 on medium access in this section, then moving up the proto- 1680 col stack to routing in the next section. In considering MAC, 1681 


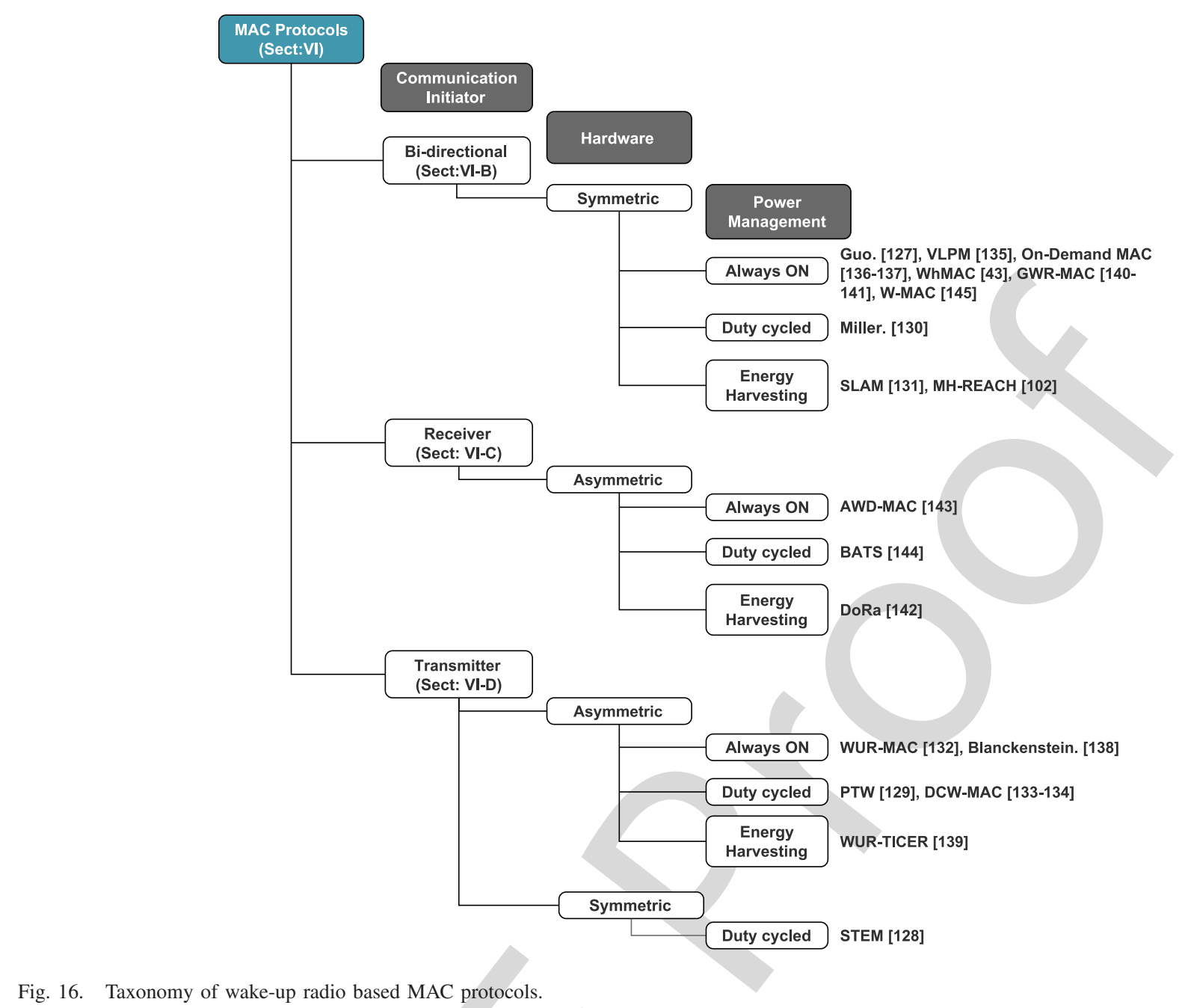

1682 we address properties both general to wireless medium access 1683 and specific to WuR. Table X summarizes the different WuR 1684 based MAC protocols designed so far while Fig. 16 organizes 1685 them into a taxonomy.

\section{A. Classification of WuR-Based Medium Access}

${ }_{1687}$ In the last decade, various MAC protocols have been 1688 proposed for wireless sensor networks. Most of these energy 1689 conservation protocols [5], [19], [23] are single-radio based 1690 and use duty cycling mechanisms. In duty cycling mode the 1691 nodes periodically wake-up to sample the channel and then go 1692 back to the sleep state. However, duty cycling MACs suffer 1693 from idle listening and waiting time that increases the data 1694 latency and power consumption (see Section I-A). Wake-up 1695 radios combat this at the hardware level, but they must also 1696 be coupled with a MAC protocol to control their use.

1697 The main contrast between traditional asynchronous MAC 1698 protocols and MACs designed for use with WuRs is that 1699 dual-radios are utilized, one of which is the extreme low1700 consumption WuR. In the former, different power management 1701 techniques are applied to the main transceiver for reducing 1702 radio-on times. The latter uses different strategies to control 1703 the secondary radio while keeping the main radio off during 1704 periods of inactivity.
MAC protocols typically divide themselves between on- 1705 demand and scheduled, with a majority of existing WuR 1706 protocols falling into the former category for flexibility and 1707 simplicity as complex, system wide schedules are not required. 1708 Further, an on-demand approach well-suits the use of the WuR 1709 as a trigger, and avoids heavy resource requirements to build, 1710 communicate, and store schedules. Below we focus on sev- 1711 eral dimensions to on-demand communication, discussing how 1712 the WuR paradigm changes their applicability w.r.t. standard 1713 wireless communication. Fig. 17 (notably not drawn to scale) 1714 illustrates different WuR based communication schemes that 1715 can be adopted for various applications. Two channels are uti- 1716 lized, the WuR channel and the main radio channel. The height 1717 of the bar symbolically represents the power consumption of 1718 the respective transceivers ( $\mathrm{WuR}$ and the main radio) in active 1719 and inactive states during different radio events while the width 1720 represents the radio on-time.

1721

The first concern we address in the taxonomy of Fig. 161722 requires identifying which pair of nodes is allocated the wire- 1723 less channel based on who is the communication initiator: the 1724 transmitter, the receiver or either (bi-directional). 1725

(i) Initiator (Transmitter): In a Transmitter-initiated pro- 1726 tocol, the node that has data to send initiates 1727 communication (Fig. 17(a)). It first sends a wake-up 1728 


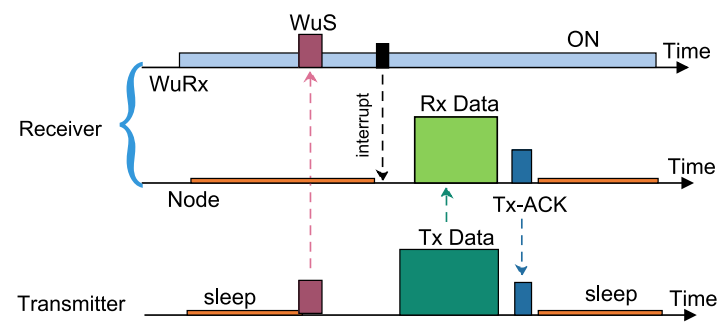

(a) Transmitter-Initiated MAC

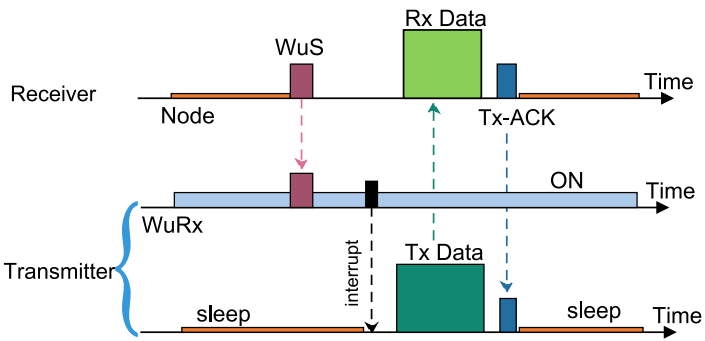

(b) Receiver-Initiated MAC

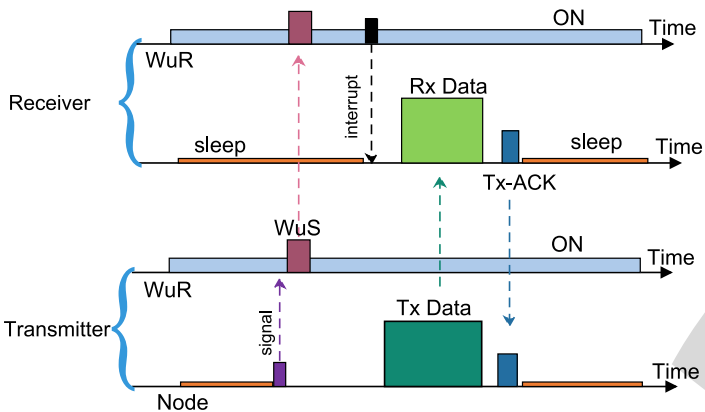

(c) Symmetric WuR System.

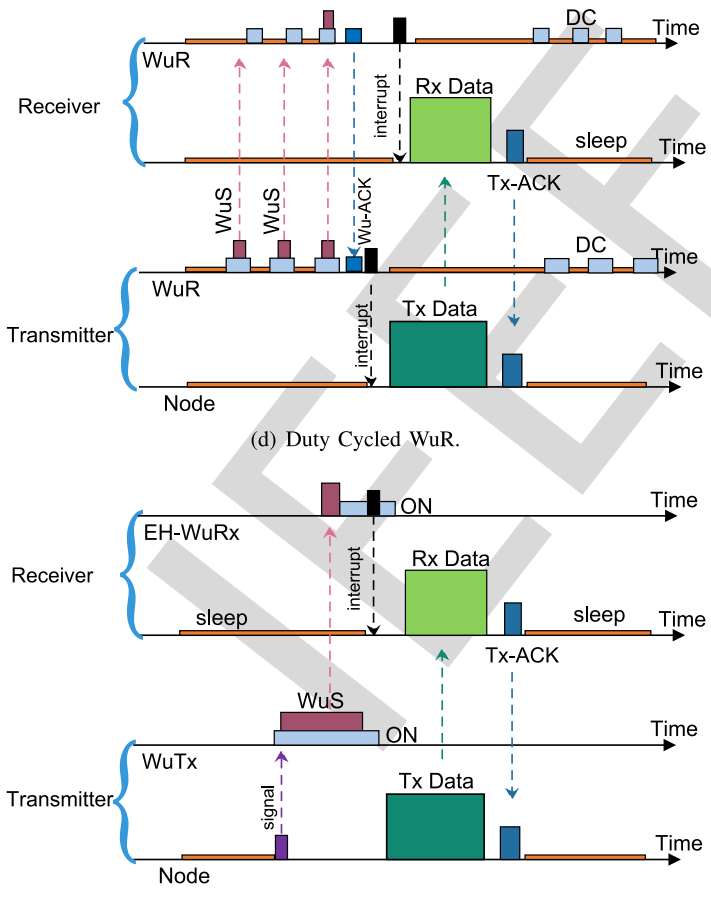

(e) Energy Harvesting WuR System.

Fig. 17. Various wake-up radio communication schemes.

signal, whose receipt triggers the receiver to wake up its main transceiver. Data is exchanged using the main transceivers followed by Tx-ACK if transmission was successful. The nodes then go back into 1732 sleep mode.

1733

(ii) Initiator (Receiver): In Receiver-initiated systems 1734 (Fig. 17(b)), the burden of starting a communication 1735 event falls to the receiver, specifically with the node, 1736 often the sink, announcing its readiness to receive data. 1737 After this announcement, it switches to receive (RX) 1738 mode and monitors the wireless channel to receive any 1739 incoming packets. If we assume the WuRx on the sender 1740 side is always active and listening, when it receives the 1741 signal it activates its main transceiver to send the data 1742 packet. The session ends when the transmit acknowl- 1743 edgment (Tx-ACK) signal arrives at the sender from 1744 the destination node, after correctly receiving the data 1745 packet. All the nodes then go back to sleep mode. This 1746 communication modality is most effective when trans- 1747 missions are infrequent, and collisions at the receiver are 1748 unlikely.

1749

(iii) Initiator (Bi-directional): In bi-directional systems, 1750 either of the nodes that want to push or pull data can ini- 1751 tiate the communication via their respective WuRs. The 1752 data packet is still exchanged between main transceivers. 1753 This setup is more suitable for enabling multi-hop 1754 communication.

1755

Thus far we have ignored the placement of the specialized 1756 WuR hardware, assuming that the non-initiator is equipped 1757 with the WuRx. Here we detail asymmetric and symmetric 1758 options.

1759

(i) Hardware (Asymmetric): If only a single hop network 1760 is required, an asymmetric scheme is possible, with the 1761 WuRx on only one side of the communication link. In a 1762 scenario with a powered sink, a Receiver-Initiated solu- 1763 tion can be used to pull data to the sink from nodes that 1764 are one-hop from the sink. The non-sink nodes must 1765 have a WuRx, allowing them to wait in a very low con- 1766 sumption state, then switching to a higher consumption 1767 only when the sink is ready to receive their data. $\quad 1768$

(ii) Hardware (Symmetric): For a multi-hop system, each 1769 node must alternately serve as receiver and transmitter, 1770 resulting in a symmetric system in which all nodes are 1771 equipped with a wake-up transceiver. Either receiver- 1772 or transmitter-initiated schemes are possible. Fig. 17(c) 1773 shows a transmitter-initiated case, in which the transmit- 1774 ter sends a wake-up signal to the receiver. The receipt of 1775 this signal triggers the activation of the main transceivers 1776 for data exchange.

1777

Next we turn to the usage of the wake-up radio itself, 1778 concentrating on how and when it is powered. There are 1779 three power management techniques that can be applied: 1780 always-ON, duty cycling the WuR or energy harvesting. $\quad 1781$

(i) Power (Always-On WuR): Typically, due to the low con- 1782 sumption of the WuRx technology, it can be constantly 1783 powered, waiting for a trigger signal. In a transmitter- 1784 initiated scenario, this minimizes the latency, as the 1785 receiver is immediately aware of the transmitter's need 1786 to initiate communication.

1787

(ii) Power (Duty Cycled WuR): To further reduce power con- 1788 sumption, the wake-up radio itself can be duty cycled 1789 

1812 taxonomy, but are nevertheless considered part of the MAC. ${ }_{1813}$ First, what information is exchanged over the WuR and sec1814 ond, whether the WuR works in the same frequency band as 1815 the main radio.

(Fig. 17(d)), meaning the WuRx is periodically put into listen mode to monitor the channel for a wake-up signal. To compensate for the sleeping times of the receiver, the WuTx must send the wake-up signals more than once, until a wake-up acknowledgment (Wu-ACK) is received from the target WuRx. When the WuRx listening period coincides with the wake-up signal transmission, the receiving node switches on its main transmitter and the main data transmission is initiated. If no Wu-ACK is received, the initiator node can re-transmit the wake-up signal. To avoid overhearing by the non-targeted nodes, the wake-up signal carries the destination address.

(iii) Power (Energy Harvesting WuR): As mentioned in Section III, in energy harvesting WuR system $(\mathrm{EH}-\mathrm{WuR})$, the WuRx is only woken up when "sufficient" energy is harvested from the wake-up signal. Fig. 17(e) illustrates the transmitter-initiated scenario where the energy from the WuS is utilized for powering up the trigger circuitry. In this scenario when there is no communication going on, the WuRx is completely switched OFF.

We next consider two, elements that we leave out of our

(i) Data (Trigger-only): The most typical use of the WuR is to trigger a higher power radio, used for communicating data. This requires very little logic on the WuR board, and minimizes hardware complexity. As mentioned previously, the trigger can be broadcast, waking up all neighboring nodes, or unicast, with the trigger containing the address of the intended recipient.

(ii) Data (WuR as main data radio): As an alternate, the low-power WuR can be responsible for all communication, i.e., for sending the wake-up signal and the data packet. The communication is still bidirectional, however, there is no main high power transceiver.

For the next option, we look at the radio itself, specifically he use of the wireless spectrum, divided into channels.

(i) Spectrum (In-Band): Few published MAC protocols address only in-band (single channel) communication, i.e., both the trigger and the data are exchanged over the same channel or frequency.

(ii) Spectrum (Out-of-Band): Multiple channels, instead, can reduce interference and increase bandwidth, but at the expense of additional coordination between senders and receivers both in time, as mentioned previously, and also across the space of the channels. In most of the WuR-MAC protocols, the bandwidth is divided into two channels: one used for control and the other for wakeup signals. Another is the data channel with higher bandwidth allocated for the main radio. For channel reservation, normally RTS/CTS handshake mechanism is performed over the control channel. The RTS/CTS frame includes a preamble, sender/receiver address, channel information for the main transceiver, and packet length. Use of out-of-band approach has following advantages.
Firstly, using different channels appropriately can lead 1848 to higher throughput. Secondly, communication on dif- 1849 ferent channels or frequency does not interfere with each 1850 other allowing multiple transmissions simultaneously, 1851 leading to fewer collisions.

In the remainder of this section, we organize our discus- 1853 sion of proposed protocols along the taxonomy of Fig. 16, 1854 first according to the communication initiator: bi-directional, 1855 receiver-initiated, and transmitter-initiated. Within each, we 1856 further sub-divide the discussion across symmetric and asym- 1857 metric hardware and different power management approaches, 1858 also offering the categorization of the protocols along the lines 1859 mentioned here.

\section{B. Bi-Directional MAC Protocols}

1861

The most populated sector for MAC protocols is bi- 1862 directional, in which any node can initiate the communication. ${ }_{1863}$ For instance, in a WBAN the traffic is normally categorized 1864 into two types: uplink where the sensing nodes can commu- 1865 nicate with the coordinator node to report urgent data and 1866 the downlink where the coordinator can send messages to 1867 the nodes. In this framework, all the nodes can be attached ${ }_{1868}$ with WuR transceivers providing bi-directional communica- 1869 tion [143]. This requires symmetric hardware on all nodes, 1870 but affords full flexibility of power management, which we 1871 detail here.

1872

1) Always ON: The MAC protocols in this category keep 1873 the low-power WuRx always ON. As such, it is able to 1874 receive the wake-up beacon immediately with reduced 1875 latency, however, the energy consumed is non-negligible. 1876 Several existing MAC protocols, VLPM [133] 1877 WhMAC [42], [104], On-Demand MAC [134], [135], 1878 and GWR-MAC [138], [139], have been proposed for 1879 the star topology, applying this schema using existing 1880 wakeup radios to WBAN. The authors assume that 1881 the wake-up beacon contains the target destination 1882 node address allowing other nodes in the network to 1883 keep their main radio in sleep state. However, all of 1884 these works ignore the fact that different physiological 1885 parameters sampled by different sensor nodes generally 1886 have significant differences in terms of traffic arrival 1887 and data rate. For instance, sensors monitoring electro- 1888 cardiography (ECG) is allocated high data rate while 1889 body temperature sensors are assigned low data rate. If 1890 the same energy saving strategy is used to cope with 1891 all of the sensor nodes, the nodes with high energy 1892 consumption rate will quickly exhaust their energy, 1893 which eventually reduce the entire network lifetime. In 1894 addition, while some of these protocols may work well 1895 in a small, single-hop network like a WBAN, they may 1896 lack in flexibility to work for more general WSNs with 1897 a large number of nodes. Guo et al. [125] proposed 1898 one of the earliest protocols using always-on WuRxs 1899 to show the benefit of bi-directional over traditional 1900 radios with duty cycling MAC. The receiver assigns the 1901 nodes with unique data channels by encoding channel 1902 information in the wake-up beacon called channel 1903 
TABLE $X$

WAKE-UP RADIO BASED MAC PROTOCOL DESIGNS

\begin{tabular}{|c|c|c|c|c|c|c|c|c|c|}
\hline No. & Protocol & Year & Initiator & Hardware & $\begin{array}{c}\text { Power } \\
\text { Management }\end{array}$ & $\begin{array}{c}\text { Information } \\
\text { Exchange }\end{array}$ & Channels & $\begin{array}{l}\text { Key } \\
\text { Novelty }\end{array}$ & Implement $^{n}$ \\
\hline 1 & Guo et al. [125] & 2001 & Bidirectional & Symmetric & Always ON & Trigger & Multiple & $\begin{array}{l}\text {-Embedding channel } \\
\text { information in WuS }\end{array}$ & Simulation \\
\hline 2 & STEM-T [126] & 2002 & Transmitter & Symmetric & Duty Cycled & Trigger & Out-of-Band & $\begin{array}{l}\text {-All neighbors } \\
\text { woken up }\end{array}$ & Simulation \\
\hline 3 & STEM-B [126] & 2002 & Transmitter & Symmetric & Duty Cycled & Trigger & Out-of-Band & $\begin{array}{l}\text {-Addressed } \\
\text { Beacon }\end{array}$ & Simulation \\
\hline 4 & PTW [127] & 2004 & Transmitter & Asymmetric & Duty Cycled & Trigger & Out-of-Band & $\begin{array}{l}\text {-Broadcast wake-up } \\
\text {-Addressing on } \\
\text { data channel }\end{array}$ & Simulation \\
\hline 5 & Miller et al. [128] & 2005 & Bidirectional & Symmetric & Duty Cycled & Trigger & Multiple & - Wake up scheduling & Simulation \\
\hline 6 & SLAM [129] & 2007 & Bidirectional & Symmetric & $\begin{array}{c}\text { Energy } \\
\text { harvesting }\end{array}$ & Trigger & Multiple & $\begin{array}{l}\text {-Energy harvesting } \\
\text { by all nodes }\end{array}$ & Simulation \\
\hline 7 & WUR-MAC [130] & 2009 & Transmitter & Asymmetric & Always ON & Trigger & Out-of-Band & $\begin{array}{l}\text {-CTS / RTS on } \\
\text { WuR channel }\end{array}$ & Simulation \\
\hline 8 & DCW-MAC [131], [132] & 2011-14 & Transmitter & Asymmetric & Duty Cycled & Trigger & In-Band & $\begin{array}{l}\text {-Single trasmitter for } \\
\text { trigger and data } \\
\text {-Separate WuRxs }\end{array}$ & Simulation \\
\hline 9 & VLPM [133] & 2011 & Bidirectional & Symmetric & Always ON & Trigger & Multiple & $\begin{array}{l}\text {-Bidirectional } \\
\text { wake up }\end{array}$ & Simulation \\
\hline 10 & On-Demand MAC [134], [135] & 2011 & Bidirectional & Symmetric & Always ON & Trigger & Multiple & $\begin{array}{l}\text {-Bidirectional } \\
\text { wake-up }\end{array}$ & Simulation \\
\hline 11 & Blanckenstein et al. [136] & 2012 & Transmitter & Asymmetric & Always ON & Trigger & In-Band & $\begin{array}{l}\text {-Node clustering } \\
\text {-TDMA on main radio }\end{array}$ & Simulation \\
\hline 12 & WhMAC [42] & 2012 & Bidirectional & Symmetric & Always ON & Trigger & Multiple & $\begin{array}{l}\text {-TDMA on } \\
\text { main radio }\end{array}$ & Simulation \\
\hline 13 & WUR-TICER [137] & 2013 & Transmitter & Asymmetric & $\begin{array}{c}\text { Energy } \\
\text { harvesting }\end{array}$ & Trigger & In-Band & $\begin{array}{l}\text {-Energy harvesting } \\
\text { by all nodes }\end{array}$ & Simulation \\
\hline 14 & GWR-MAC [138], [139] & 2014 & Bidirectional & Symmetric & Always ON & Trigger & Multiple & $\begin{array}{l}\text {-Bidirectional } \\
\text { wake up }\end{array}$ & Simulation \\
\hline 15 & MH-REACH [100] & 2015 & Bidirectional & Symmetric & $\begin{array}{c}\text { Energy } \\
\text { harvesting }\end{array}$ & Trigger & Out-of-Band & -Passive RFID & Testbed \\
\hline 16 & DoRa [140] & 2015 & Receiver & Asymmetric & $\begin{array}{c}\text { Energy } \\
\text { harvesting }\end{array}$ & Trigger & Out-of-Band & $\begin{array}{l}\text {-Energy harvesting } \\
\text {-Base station wakes } \\
\text { up the neighbors }\end{array}$ & Simulation \\
\hline 17 & AWD-MAC [141] & 2015 & Receiver & Asymmetric & Always ON & $\begin{array}{l}\text { Set of } \\
\text { Triggers }\end{array}$ & In-Band & $\begin{array}{l}\text {-Wake up } \\
\text { multiple neighbors }\end{array}$ & Simulation \\
\hline 18 & BATS [142] & 2016 & Receiver & Asymmetric & Duty Cycled & Trigger & Out-of-Band & -Supports Mobility & Testbed \\
\hline 19 & W-MAC [143] & 2017 & Bidirectional & Symmetric & Always ON & Trigger & Out-of-Band & $\begin{array}{l}\text {-Bidirectional wake-up } \\
\text {-Addressed beacon } \\
\text {-Supports multi-hop }\end{array}$ & Simulation \\
\hline
\end{tabular}

based local addressing scheme. The transmitting node captures this information via its WuRx and switches its data radio to receiver's channel after activating the main node. Through the simulation of their protocol in broadcast mode, the authors showed that power reduction of $10 \sim 100$ times can be achieved with always-on WuRxs compared to duty cycled main radio solutions. To target real WSN applications, W-MAC [143] was proposed for multi-hop network in which nodes alternately act as senders and receivers. W-MAC takes advantage of secondary always-on WuR that is attached to the main mote acting as the communication initiator. Whenever a node has data to send, either generated by the upper layers of the protocol stack or forwarded by neighboring nodes, W-MAC first transmits a wake-up beacon containing the destination node address. To avoid collisions, the WuR and the main radio use different channels for wake-up beacon and data packets. Using simulations with two different routing protocols, W-MAC illustrated that 1923 WuR technology has the potential to offer significant 1924 energy savings without compromising on reliability 1925 and latency.

2) Duty cycled: Another bi-directional communication is 1927 proposed by Miller and Vaidya [128]. To avoid costly 1928 full wake-ups, the sensor nodes schedule a triggered 1929 wake-up with a receiver. This schedule is calculated 1930 by the sink node based on the previous traffic patterns 1931 and is then disseminated to the network. Each node in 1932 the network knows their next wake-up time and when 1933 there is nothing to receive, the WuR is switched into 1934 duty cycling mode until the next wake-up cycle. The 1935 proposed idea is compared to STEM [126] and the simu- 1936 lations show significant reduction in the delivery latency. 1937 Nevertheless, schedule sharing requires tight synchro- 1938 nization at the receiver side leading to extra energy over- 1939 head to overcome clock drifts. The authors also assume 1940 that all the nodes share the same wake-up channel 1941 
without specific node addressing, thus triggering all the nodes.

3) Energy harvesting: MH-REACH is a MAC protocol designed for passive RFID-based WuR systems supporting multi-hop wake-up sensor networks [100]. In it, the WuTx on the sink wakes up all nodes in its vicinity. Any node that was woken up offloads its data to the sink, and, if it is a multi-hop node, it also transmits a wake-up signal to wake up other nodes within its transmission range. If it is an edge node, after transmitting its data to the sink, it returns to the sleep state until the next wake-up event. Although this protocol supports a multi-hop network, the passive devices require wake-up signals of longer duration (between 5s-10s) to accumulate enough energy to fully power-up the circuitry. Therefore, applications must trade-off maximum wake-up range and node lifetime. In addition, due to its broadcast nature of the $\mathrm{WuS}$, all the nodes within 1-hop are activated, thus contributing to overhearing overhead. A similar energy harvesting based MAC protocol (SLAM) has been proposed in [129]. In SLAM, a few nodes are assigned as guard nodes that monitor the traffic between hops to detect malicious nodes. During periods of inactivity the guard nodes are put into sleep mode and switched on when required via passive WuRxs. Through experiments authors have shown that listening energy can be reduced by to 30-129 times using WuRs while providing a high level of network security.

\section{C. Receiver-Initiated MAC Protocols}

1971 To increase throughput and to shift the burden of energy 1972 consumption from the sender to receiver, some authors 1973 have proposed receiver-initiated WuR-MAC protocols. Their 1974 design is inherently asymmetric, and the full range of power 1975 management techniques are applicable.

1) Always ON: To extend the life of sensing nodes, AWD-MAC [141] utilizes the receiver-initiated scheme but employs a single channel for communication. Different from the traditional receiver-initiated cycled receiver (RICER) where only one common broadcast beacon is sent, AWD-MAC first sends a set of wakeup beacons in sequence to wake-up multiple neighbors for neighbor discovery. The nodes then reply using random slots with their node IDs and respective data rates. Subsequently, the coordinator node creates a neighbor table to query each node in an asynchronous fashion. AWD-MAC claims that the collisions are removed as only one transmitter node is allowed to send its data at a given time while sharing the same channel. Nonetheless, collisions do occur during the neighbor discovery phase when AWD-MAC sends the broadcast beacon to detect new nodes.

2) Duty cycled: The first mobility-based WuRx system using the receiver-initiated paradigm has been proposed in the BATS project [144]. The authors have investigated the potential of ultra-low power WuRs carried by bats to monitor encounters between individuals and to track their routes at high spatial and temporal res- 1998 olution [142], [145], [146]. Due to limited available 1999 energy, the wake-up receivers are duty cycled. To sup- 2000 port multiple mobile nodes and to prevent the colli- 2001 sions at the receiver side, the ground node uses Time 2002 Division Multiple Access (TDMA)-like communication 2003 slots with guard intervals between slots. The communi- 2004 cation between the mobile nodes is not synchronized. 2005 When the mobile node enters the communication range 2006 of the ground node, the latter sends a wake-up beacon. 2007 Upon successful wakeup, the mobile node offloads the 2008 data within its assigned slot. Due to the high mobil- 2009 ity of the bat nodes, no carrier sensing techniques 2010 are performed prior to transmission allowing mobile 2011 nodes to send data before exiting the transmission 2012 range. Therefore, if multiple mobile nodes are within 2013 the receivers vicinity, data collisions may occur and the 2014 packets can be lost.

2015

3) Energy harvesting: DoRa [140] offers a WuR-MAC 2016 protocol that builds upon the foundation of the receiver- 2017 initiated paradigm for the realization of energy harvest- 2018 ing in one hop networks. In the proposed mechanism, 2019 no channel reservation or packet acknowledgments are 2020 transmitted. The nodes answer to the base station by 2021 directly sending the data packet. DoRa also provides 2022 out-of-band support and node addressing. However, sim- 2023 ilar to MH-REACH, a strong wake-up signal is required 2024 in order to harvest enough energy to activate the nodes 2025 leading to high data latency.

\section{Transmitter-Initiated MAC Protocols}

2027

We next consider transmitter-initiated MAC proto- 2028 cols where each node chooses its transmission schedule 2029 autonomously. In general, this approach puts the energy con- 2030 sumption burden for transmission on the sender, with a much 2031 lighter load on the receiver. Both asymmetric and symmet- 2032 ric approaches are possible, and multiple power management 2033 techniques have been applied.

We begin with asymmetric:

2034

1) Always ON: A transmitter-initiated MAC protocol leveraging always-on WuRxs is proposed by 2037 Mahlknecht and Durante [130]. WUR-MAC is based 2038 on multi-channel principle and uses RTS and CTS 2039 handshake mechanism. The sender node first transmits 2040 the request-to-send packet for selecting the appropriate 2041 receiver. The intended node then replies with clear-to- 2042 send packet and triggers its main radio for data reception 2043 at higher bandwidth. WUR-MAC supports both point- 2044 to-point and broadcast communication. Using channel 2045 reservation reduces collisions but may impact on the 2046 data latency as the transmission is blocked until CTS 2047 is successfully exchanged. Energy efficient node clus- 2048 tering using WuRxs for WBAN sensors with similar 2049 readings is presented in [136]. To eliminate idle lis- 2050 tening and channel contention, an always-on WuRx is 2051 attached to a main radio that utilizes TDMA scheme. 2052 To achieve clustering, the relevant data information is 2053 
encoded in the WuTx's data pattern. The idea is to reduce energy consumption by reducing the number of data packets through clustering nodes with similar sensor readings and allowing only the cluster head to forward data to the sink. This protocol is only tested using simulations where the wake-up addressing mechanism is used to trigger nodes according to the data they have sensed.

2) Duty cycled: Similar to STEM-T, Yang and Vaidya [127] propose a Pipelined Tone Wakeup (PTW) scheme that uses two different radio channels, one for data and one for tone detection. In PTW, the WuRx is duty cycled. When a node has packets to send, it transmits a tone on the wakeup channel and sends the notification packet on the data channel to specify the target node. As the wake-up tone is broadcast, any node within the transmission range of sender will be awakened. From the point of view of application scenarios for opportunistic networking, such an approach could grant fast wake-up in dense and multi-hop scenarios while reducing end-to-end latency, but could be less energy efficient. Analogous to STEM and PTW, the work in [131] and [132] also duty cycles the WuRx statically, but uses in-band approach for communication. In DCW-MAC, the main radio is used for both sending the wake-up beacon and the data, but the authors add dedicated, secondary low-power radio, acting as a WuRx, operating in the same frequency band. The authors through analytical models derive the optimal sleep and listen time for a duty cycled WuRx and compare these models to a non-WuR based system. However, the analysis assumes perfect detection of wake-up signals and energy consumed due to collisions is ignored in the derivation of optimal timing. In addition, the main radio also acts as a wake-up transmitter, hence, frequent switching between RX and TX mode may result in extra energy consumption.

3) Energy harvesting: Le et al. [137] have proposed the WUR-TICER MAC protocol that operates by harvesting energy from the ambient environment. The protocol is based on nano-watt WuRx proposed in [147] embedded with an energy harvesting WSN node. Whenever the transmitter has a packet, it broadcasts a wake-up beacon (WUB) indicating to other receivers that it is ready to send. Since the main radio has been used as a WuTx, WUR-TICER utilizes the same channel for sending the WUB and the data packet. As a result, WUR-TICER achieves a lower packet reception rate than the non-WuR model since the WUB collisions are frequent when two or more transmitter nodes wake-up at the same time and try to send a WUB to the base station. Moreover, the WuR is only simulated in a single-hop energy harvesting WSN with a continuous energy source.

Moving on to symmetric protocols, we find only one:

1) Duty cycled: STEM [126] is one of the first transmitterinitiated protocols that separates the data transmission channel from the wake-up channel by using a dual radio approach on separate frequency bands. Both the radios are high power radios while one of them acts as a WuR. ${ }_{2112}$ Two variants exist in STEM. In STEM-T, a tone is ${ }_{2113}$ sent which wakes up all the nodes in the neighborhood. 2114 STEM-T resembles the traditional preamble sampling ${ }_{2115}$ approach but moves the data transmission to a sepa- 2116 rate channel. In STEM-B, a wake-up beacon is used as 2117 a preamble that includes the address of the destination 2118 node and the sender. A node thus can determine whether ${ }_{2119}$ it is the intended receiver or not and the non-target nodes 2120 can go back to sleep earlier. Moreover, STEM uses a 2121 regular high power radio as a WuR to achieve the same ${ }_{2122}$ coverage as the main radio. Duty cycling is applied to 2123 the WuR while the data radio is switched off unless 2124 required. However, both radios are high power radios 2125 and the power consumption is not reduced.

\section{E. Summary}

2127

To make the wake-up radio based system feasible and 2128 energy-efficient, it requires careful design of energy-efficient ${ }_{2129}$ protocols. The MAC layer plays a crucial role in coordinating 2130 how nodes share the common broadcast channel. The main 2131 role of this layer is to prevent simultaneous transmissions and ${ }_{2132}$ data packet collisions at the same time granting energy effi- 2133 ciency, low channel access delays and ensuring fairness among 2134 the nodes in the network.

2135

So far various asynchronous MAC protocols have been ${ }_{2136}$ proposed for WuRs to extend the network lifetime and to 2137 increase reliability and throughput. Within this, different com- 2138 munication and power management techniques have been ${ }_{2139}$ employed. For reducing latency, active WuR based MAC pro- 2140 tocols have been proposed. This allows fast response and long ${ }_{2141}$ communication as the radios are always on. Although this 2142 technique provides many advantages, it is less energy efficient 2143 as WuRs are always on and dissipate energy. To make this 2144 approach effective, energy-efficient WuR hardware design is 2145 required. Some works have proposed energy harvesting based 2146 MACs and use energy from the wake-up signal itself. The 2147 reason is to extend the node lifetime and to only turn on the 2148 device upon detection of the valid signal. Other sets of proto- 2149 cols have duty cycled the WuRs. Although this reduces power 2150 demand but encounters same problems as traditional MACs 2151 such as increased latency.

2152

To enable on-demand communication, asynchronous WuR- 2153 MAC protocols have mostly adopted transmitter initiated 2154 probing for data transmission. The nodes are only equipped 2155 with the WuRxs (asymmetric) while the main radio is utilized 2156 as the transmitter. This allows only one-way communication 2157 and does not exploit the full potential of WuRs. For the 2158 WuR based system to be effective and applicable for vari- 2159 ous applications bi-directional MACs are more suitable. The 2160 main radio-activity is reduced by exchanging control informa- 2161 tion over the WuRs. This information can include channel or ${ }_{2162}$ frequency reservation data, which otherwise would have been ${ }_{2163}$ exchanged over the main radio.

2164

As the number of WuR-MAC protocols grows, there are 2165 still many open questions such as the different performance 2166 they offer when applied to realistic applications. Most of ${ }_{2167}$ 
2168 the protocol evaluation concentrates primarily on simulation 2169 results and does not include any results from hardware imple2170 mentations or testbeds. Moreover, their implementation relies 2171 on custom-design software limiting the reproducibility of the 2172 obtained results. Some of these works have quantified the ben2173 efits of using WuRs in terms of energy consumption through 2174 in-lab power measurements, but do not evaluate other relevant 2175 metrics, such as latency and end-to-end data reliability. While 2176 some of these protocols may work well in a small, single-hop 2177 network like a WBAN, it may lack in flexibility to work for 2178 more general WSNs with a large number of nodes.

\section{VII. ROUTING PROTOCOLS UTILIZING WURS}

2180 In typical WSNs, hundreds or thousands of sensor nodes are 2181 scattered or placed throughout a large area. Each sensor has 2182 the capability to communicate, collect, and route data to other 2183 nodes or back to the base station. Since, not all of these sensors 2184 are in range of the base station, data is routed in a multi-hop 2185 fashion. Over the last several decades, a plethora of routing 2186 strategies have been proposed for WSNs [25]-[28]. However, 2187 most of these studies are based on single radio architecture. 2188 The scenario changes when routing is done over WuRs due to 2189 the network topology induced by it.

2190 One of the challenges of introducing a WuR as a new com2191 ponent to an existing node with wireless communication is the 2192 mismatch between the ranges. By nature, WuR technology has 2193 shorter ranges, prohibiting a wake-up signal from triggering a ${ }_{2194}$ distant node, despite the ability of the higher power radio to 2195 effectively reach it. This introduces new challenges for tradi2196 tional routing protocols. In particular, for WuR based systems, 2197 packets need to be routed through longer paths than those of 2198 the main radio. This affects the data latency as well as the 2199 network lifetime. For applications with stringent consump2200 tion requirements, this may not be acceptable. To mitigate 2201 this, several WuR based routing protocols have been devel2202 oped for flooding, multi-hop data collection and dissemination. 2203 Table XI summarizes the WuR-based routing protocols that 2204 we survey while Fig. 18 arranges them in a taxonomy based 2205 on whether they address only the routing layer or are also 2206 cross-layer.

\section{A. Routing-Only Protocols}

2208 Existing routing-only protocols exploiting wake-up radios 2209 can be classified into three categories: topology-based, load 2210 balancing, or tree-based.

2211 1) Topology Based: Under this category, every node in 2212 the network maintains routing information such as its end2213 to-end distance to the sink and also the next hop to reach the 2214 sink. This information is usually obtained by the sink using a 2215 network wide dissemination of control messages. To forward 2216 a packet towards the sink, the node chooses the neighbor that 2217 has the shortest path as the next forwarder.

2218 Stathopoulos et al. [148] present a topology control mecha2219 nism for establishing the end-to-end paths in a WSN using the 2220 dual-radio system. Each node uses its low bandwidth wake-up 2221 radio to request an end-to-end path information to the destina2222 tion nodes from the central topology controller. The novelty of this work is to use multiple short WuR hops to achieve a 2223 single, long higher power hop by the main radio. This protocol 2224 is based on an out-of-band paradigm and supports multi-hop 2225 networks. Latency is the main issue here as path discovery 2226 using low data rate networks can be time-consuming. Since 2227 the topology controller is centralized, this can lead to a single 2228 point of failure, crippling the entire network.

2229

The concept of semantic addressing using WuRs, in which 2230 a pool of multiple WuRx addresses is assigned to a node and 2231 dynamically updated based on its status, has been recently 2232 proposed [39]. A dedicated WuRx-enabled communication 2233 stack called FLOOD-WUP exploiting selective wake-ups 2234 and dynamic address assignment is implemented to enhance 2235 system performance. FLOOD-WUP enables transmission of 2236 commands from the sink to the sensor nodes in a reliable and ${ }_{2237}$ energy efficient way. Comparing FLOOD-WUP against tradi- 2238 tional flooding protocol has shown that nodes using FLOOD- 2239 WUP for interest dissemination are 4\% energy efficient and 2240 require less energy to achieve full network coverage. 2241

2) Load Balancing: Routing protocols designed for load 2242 balancing not only select the shortest paths towards the des- 2243 tination but can also consider the available energy of the 2244 nodes in the path in an attempt to extend network lifetime. 2245 The routing load is distributed over multiple paths in the 2246 network to improve packet latency and to minimize dropping 2247 packets.

2248

To achieve reliable end-to-end data delivery, a load- 2249 balancing, and optimized data flow communication routing 2250 tree is proposed by Vodel et al. [149]. WRTA is a lightweight 2251 routing protocol for data-centric WSN environments that 2252 combines complex route path calculations and topology opti- 2253 mization mechanisms for asynchronous communications. In 2254 WRTA, the burden of energy consuming calculations such as 2255 maintaining routing path and network status is shifted from the 2256 sensing nodes to the sink. For load-balancing and route opti- 2257 mization, the shortest path is selected for nodes with a large 2258 amount of data depending on the energy level, QoS parameters 2259 and bandwidth of the nodes. WRTA was analyzed using both 2260 software and hardware experiments. It was observed that for 2261 a network with the depth of 3-hops, the proposed routing pro- 2262 tocol experiences high packet loss when the number of packet 2263 generation increases to 7 packets per node/min. 2264

3) Tree-Based: In tree-based routing, nodes form a tree- 2265 like hierarchy with the sink node as the root. Each node 2266 (child) at the particular depth of the tree transmits data to 2267 a node (parent) in the upper level of the hierarchy. This 2268 ensures data transmission in parallel and reduces packet 2269 latency significantly.

2270

Recently, Gnawali et al. [150] extended the Collection Tree 2271 Protocol (CTP), the de facto standard for data collection in 2272 WSN to work with nodes coupled with WuRs [151]. CTP- 2273 WUR utilizes WuRs to relay wake-up requests and reduces 2274 end-to-end data latency, thereby, extending the achievable 2275 wake-up range. CTP-WUR can handle both broadcast and 2276 unicast packets. It has been shown through simulations that 2277 CTP-WUR performs better, obtaining latencies lower than 2278 tens of microseconds and is highly reliable compared to the 2279 standard CTP. 
TABLE XI

WAKE-UP RADIO BASED Routing PROTOCOLS

\begin{tabular}{llcccccl}
\hline No. & Protocol & Year & Path Request & Hardware & Addressing & Topology & Implementation \\
\hline 1 & EAR [152] & 2002 & Source & Symmetric & ID-based & Distributed & Simulation \\
\hline 2 & LESOP [153] & 2007 & Source & Symmetric & ID-based & Distributed & Simulation \\
\hline 3 & Stathopoulos et al. [148] & 2007 & Source & Symmetric & ID-based & Centralized & Testbed \\
\hline 4 & WRTA [149] & 2012 & Sink & Symmetric & ID-Based & Centralized & Testbed \\
\hline 5 & FLOOD-WUP [39] & 2014 & Sink & Symmetric & ID-Based & Distributed & Simulation \\
\hline 6 & CL-RW [154] & 2014 & Source & Symmetric & ID-Based & Distributed & Testbed \\
\hline 7 & ALBA-WUR [54] & 2015 & Source & Symmetric & ID-Based & Distributed & Simulation \\
\hline 8 & ZIPPY [37] & 2015 & Sink & Symmetric & ID-Based & Distributed & Testbed \\
\hline 9 & CTP-WUR [151] & 2016 & Source & Symmetric & ID-Based & Distributed & Simulation \\
\hline 10 & OPWUM [155] & 2016 & Sink & Symmetric & ID-Based & Distributed & Simulation \\
\hline 11 & T-ROME [156] & 2017 & Source & Symmetric & ID-Based & Distributed & Testbed \\
\hline
\end{tabular}

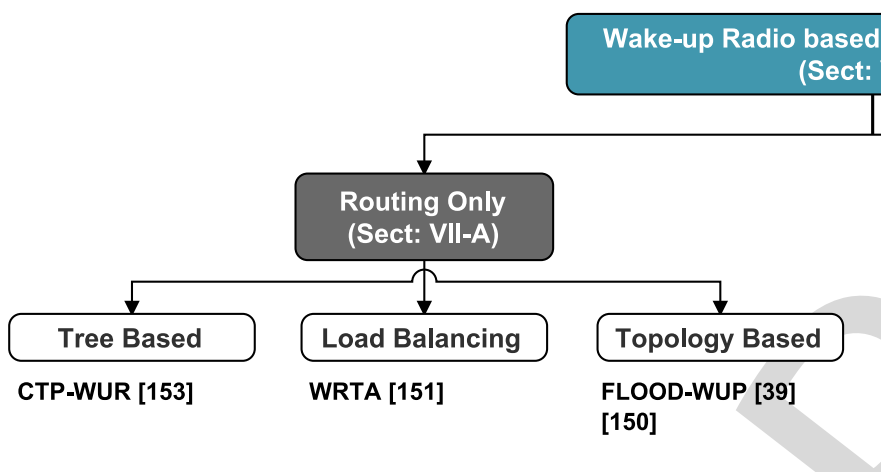

Fig. 18. Taxonomy of wake-up radio based routing protocols.

\section{B. Cross-Layer Protocols}

2282 The protocols discussed so far were individually developed 2283 for a single layer of the stack, i.e., MAC, Network, Transport, 2284 and Physical. While they exhibit good performance in terms 2285 of the metrics related to a single layer, they are not jointly 2286 optimized to maximize overall network performance while 2287 reducing energy expenditure. Therefore, a cross-layer design 2288 presents a promising alternative by streamlining communi2289 cation between layers and providing the response based on 2290 a complete view of the stack, increasing system utility and 2291 energy efficiency.

2292 1) Energy-Aware: The main objective of energy-aware 2293 routing protocols is to extend the network lifetime by choosing 2294 optimal paths. These paths are chosen depending on the energy 2295 budget so that no single path depletes its energy quickly. 2296 Rotating among paths leads to increased network lifetime as 2297 energy is dissipated equally among all the nodes.

2298 A cross-layer energy aware routing (EAR) protocol using 2299 WuRs [152] uses sub-optimal paths to provide substantial 2300 gains in network lifetime. In EAR, the MAC layer is respon2301 sible for keeping the lists of all its neighbors and metrics such 2302 as the neighbor's position and the energy required to reach 2303 it. Then, this list is accessed by the network layer to make 2304 decisions regarding packet routing. The energy level informa2305 tion is used as a weight factor when routing the data, avoiding 2306 the paths with less residual energy. Finally, to send data the
MAC layer transmits a wake-up signal on the broadcast chan- 2307 nel, modulating the address of targeted node with the wake-up 2308 signal. Even though this method takes energy into account, it 2309 does not consider end-to-end latency. Moreover, this protocol 2310 has only been evaluated through simulations.

2311

OPWUM [155] offers another opportunistic cross-layer 2312 MAC protocol leveraging WuRxs for selecting the best 2313 receiver among its neighboring nodes using energy as a met- 2314 ric. To overcome collisions between wake-up beacons, a clear 2315 channel assessment (CCA) is performed using the WuTx. 2316 Thereafter, an RTS-CTS is exchanged between the WuTx and 2317 WuRx before sending any data packets via the main radio. 2318 One of the features of OPWUM is that all the next hop relay 2319 selection phase is carried out using wake-up beacons only. 2320 Nonetheless, this proposed protocol has not been tested using 2321 real experiments.

2322

Unlike classical approaches, Low Energy Self-Organizing 2323 Protocol (LESOP) [153] presents a cross-layer architecture 2324 where both Application and MAC layers collaborate directly 2325 while Transport and Network layers are excluded to simplify 2326 the protocol stack. Inter-node communications are done by 2327 exchanging packets and busy tones. The main radio is respon- 2328 sible for handling all data packets while the busy tones are 2329 sent using the secondary low power wake-up radios. This 2330 protocol is proposed for target tracking applications in large 2331 wireless sensor networks. Similar to EAR, this protocol does 2332 
2333 not investigate the importance of system delay and is tested 2334 in simulations only.

2335 2) Geographic: In geographic routing protocols, the data 2336 packet is routed towards the destination region using geograph2337 ically informed neighbor selection heuristics. The key concept 2338 is to collect data from the selected region rather than sending 2339 it through the whole network hop by hop.

2340 Spenza et al. [54] proposed ALBA-WUR, a cross-layer 2341 solution for data collection exploiting semantic node address2342 ing features of WuRx to implement complex relay selection 2343 policies. For data routing and path selection, the protocol 2344 relies on ALBA-R, a cross-layer geographic protocol that fea2345 tures the integration of awake/sleep schedules, MAC, routing, 2346 load balancing, and back-to-back packet transmissions [157]. 2347 Simulation results concerning average end-to-end data latency 2348 show that the use of WuR technology together with ALBA-R 2349 is effective for cutting down the time needed to deliver pack2350 ets to the destination. However, this delay is dependent on the 2351 data rate used to transmit wake-up signals.

2352 T-ROME, a cross-layer routing protocol that supports 2353 multi-hop communication, is presented in [156]. At the MAC 2354 layer, T-ROME uses RTS/CTS messages to reduce packet 2355 collisions over the $\mathrm{WuR}$. At the network layer, the data for2356 warding mechanism of T-ROME is similar to ALBA-WUR 2357 but does not flood the whole network. In T-ROME the next 2358 hop node is chosen dynamically using link quality estimation 2359 over the WuRs to determine if the relay node is within the 2360 wake-up range. If so, the data is directly sent to that particular 2361 node without passing from each child to its parent. Therefore, 2362 T-ROME saves energy by skipping nodes during data trans2363 mission. Using small scale testbed, authors have shown that 2364 T-ROME outperforms CTP-WUR in terms of number of hops 2365 required to reach the sink with reduced latency and power 2366 consumption.

2367 3) Flooding Based: In this category, the node that has data 2368 communicates it to everyone else in the network using flood2369 ing. Multiple copies of the incoming packets are sent by the 2370 nodes that are in the broadcast domain which they forward to 2371 their neighbors. This technique generates a huge amount of 2372 redundant traffic. However, it does not require costly topology 2373 maintenance and route discovery procedures.

2374 A practical application of ultra-low power sub-GHz WuR 2375 is presented by Sutton et al. [37]. ZIPPY is a cross-layer 2376 protocol that provides on-demand network flooding for the 2377 multi-hop network through the use of ultra-low power wake-up 2378 receivers equipped at each node, albeit with reduced per-hop 2379 range compared to using high-power transceivers. The ZIPPY 2380 protocol features asynchronous network wake-up, neighbor2381 hood time synchronization, bit-level data dissemination and 2382 carrier frequency randomization leveraging low complexity ${ }_{238 з}$ WuRs. Using ZIPPY reduces the entire network flooding time 2384 while maintaining end-to-end latency of only a few microsec2385 onds. As in its current implementation, ZIPPY does not 2386 address the false wake-ups making it susceptible to erroneous 2387 network wide wake-up.

2388 Cross-layer Radio Wake (CL-RW) [154] builds on the 2389 transmitter-initiated paradigm by coordinating the wake-up 2390 beacon transmissions. The proposed mechanism uses an 2391 asynchronous scheduler for controlling its WuR, which is a cross-layer information from the MAC layer, to form an oper- 2392 ation cycle. This cycle is a network-level duty cycle that is 2393 built on top of the duty cycles of individual nodes. Instead 2394 of transmitting wake-up beacons independently, each WuTx 2395 transmits during its allocated schedule. Therefore, the beacon 2396 transmissions in a network are coordinated to form a multi-hop 2397 path like a pipeline and the waiting time in each hop is signif- 2398 icantly reduced. Furthermore, a node that has generated data 2399 can keep the radio off to save additional power. The proposed 2400 idea is compared to AS3-MAC [158] and the experiments 2401 show significant reduction in the power consumption.

\section{Summary}

This section has provided a classification of WuR based 2404 routing protocols, including also cross-layer approaches. Most of 2405 these studies have shown that by combining wake-up capabilities 2406 with selective addressing and routing over WuRs, node lifetimes 2407 can be extended to decades while achieving data latencies 2408 comparable to networks that only use the single main radio. 2409

Most of the routing protocols discussed in this section 2410 assume static networks where the sensor nodes and the base 2411 station are stationary. An interesting issue to look into will 2412 be consideration of node mobility. For diverse applications 2413 of WuRs such as smart city or transportation, routing proto- 2414 cols for mobile WSNs will be beneficial to provide real-time 2415 delivery and wider coverage. Routing messages in a mobile 2416 scenario is challenging since route stability becomes an impor- 2417 tant optimization factor, in addition to bandwidth and energy. 2418 Use of wake-up radios for mobility purposes requires opti- 2419 mization of transmitter operation, such as the number and 2420 time interval over which to transmit wake-up beacons so that 2421 they are correctly received by the low power wake-up receiver 2422 for controlling main radio operation as proposed in [159]. 2423 Novel routing algorithms are needed to handle the overhead of 2424 mobility and topology changes in such an energy-constrained 2425 environment.

2426

Multichannel routing protocols have recently gained pop- 2427 ularity in the context of WSNs, due to their ability to be 2428 resilient against interference and collision, providing a signif- 2429 icant performance benefit over a purely static approach. Such 2430 protocols involve various challenges such as channel selection, 2431 hidden terminal problem, and channel hand-over. Thus, rout- 2432 ing for multi-channel WSNs over wake-up radios needs to be 2433 further studied.

2434

Network security is another aspect that needs to be consid- 2435 ered. Routing protocols must be robust against eavesdropping 2436 and malicious behavior. An attempt to address this using 2437 wake-up radios has been made in [160].

2438

Finally, most of the routing protocols that exploit wake-up 2439 radios for the WSNs have been evaluated principally through 2440 simulations. To assess the real benefit and the performance of 2441 these protocols, thorough testing in real environments with a 2442 large network is essential.

Over the decades, the application of WSN has increased, 2445 spanning from monitoring natural phenomena such as 2446 
TABLE XII

WAKE-UP RADIO BASED APPLICATION REQUIREMENTS

\begin{tabular}{|c|c|c|c|c|c|c|c|c|}
\hline Applications & Range & Lifetime & $\begin{array}{c}\text { Mode of } \\
\text { Data Collection }\end{array}$ & $\begin{array}{l}\text { Network } \\
\text { Type }\end{array}$ & Latency & $\begin{array}{l}\text { Data } \\
\text { Rate }\end{array}$ & Addressing & $\begin{array}{l}\text { Power } \\
\text { Source }\end{array}$ \\
\hline $\begin{array}{l}\text { WBAN } \\
\text { Implantable devices }\end{array}$ & - & ++ & $\begin{array}{l}\text { Event-driven } \\
\text { On-demand }\end{array}$ & Star/Single-hop & - & ++ & Yes & Active \\
\hline $\begin{array}{l}\text { Smart City } \\
\text { Infrastructure monitoring } \\
\text { Environment monitoring }\end{array}$ & ++ & + & $\begin{array}{l}\text { Event-driven } \\
\text { On-demand }\end{array}$ & $\begin{array}{l}\text { Node-to-node } \\
\text { Multi-hop } \\
\text { Mobile }\end{array}$ & - & + & Yes & $\begin{array}{l}\text { Active } \\
\text { Passive }\end{array}$ \\
\hline $\begin{array}{l}\text { Smart Metering } \\
\text { Utility monitoring }\end{array}$ & + & + & On-demand & $\begin{array}{l}\text { Node-to-node } \\
\text { Mobile }\end{array}$ & - & & Yes & Active \\
\hline $\begin{array}{l}\text { Wildlife Monitoring } \\
\text { Contact detection }\end{array}$ & ++ & + & $\begin{array}{l}\text { Event-driven } \\
\text { Periodic }\end{array}$ & $\begin{array}{l}\text { Node-to-node } \\
\text { Multi-hop } \\
\text { Mobile }\end{array}$ & - & & Yes & Active \\
\hline Surveillance & ++ & ++ & Event-driven & $\begin{array}{l}\text { Star } \\
\text { Multi-hop }\end{array}$ & & & Yes & Active \\
\hline Indoor Localization & + & ++ & $\begin{array}{l}\text { Event-driven } \\
\text { On-demand }\end{array}$ & $\begin{array}{l}\text { Star } \\
\text { Multi-hop } \\
\text { Mobile }\end{array}$ & & & Yes & Active \\
\hline Asset Tracking & + & ++ & $\begin{array}{l}\text { Event-driven } \\
\text { On-demand } \\
\text { Periodic }\end{array}$ & $\begin{array}{l}\text { Star } \\
\text { Multi-hop } \\
\text { Mobile }\end{array}$ & + & - & Yes & $\begin{array}{l}\text { Active } \\
\text { Passive }\end{array}$ \\
\hline Wearables & - & ++ & $\begin{array}{l}\text { Event-driven } \\
\text { On-demand }\end{array}$ & $\begin{array}{l}\text { Star } \\
\text { Node-to-node }\end{array}$ & & ++ & Yes & Active \\
\hline Smart Grid & + & ++ & $\begin{array}{l}\text { Event-driven } \\
\text { On-demand }\end{array}$ & $\begin{array}{l}\text { Star } \\
\text { Multi-hop }\end{array}$ & - - & + & Yes & $\begin{array}{l}\text { Active } \\
\text { Passive }\end{array}$ \\
\hline Requirement Importance & - Low & - - Very low & + High & ++ Very high & & & & \\
\hline
\end{tabular}

2447 temperature and humidity to personal health. With the pro2448 liferation of low power and cheap semiconductors, WSNs are 2449 expected to gain even more popularity [2].

2450 With the understanding of the ultra-low power WuR built in 2451 the previous sections, we now briefly discuss multiple emerg2452 ing application scenarios that can take advantage of it. We then 2453 map the different prototypes and protocols suitable for each 2454 application. Table XII offers an overview while the remainder 2455 of this section provides details.

\section{A. Wireless Body Area Network (WBAN)}

2457 Wireless body are networks (WBANs), find applicabil2458 ity in medical applications and thus require high reliability. 2459 To support a variety of applications on or inside the body, 2460 systems must have low power consumption and support vari2461 able data rates [161]. As an example of the latter, a glucose 2462 level monitor requires less than $1 \mathrm{kbps}$ while an ECG can 2463 reach 192 kbps [161]. Further, WBAN communication can 2464 be periodic, event-driven, e.g., triggered by detection of an 2465 alert condition, or on-demand, e.g., in response to an external 2466 request by a clinician to retrieve saved data.

2467 WuR technology can be applied in two principle ways. First, 2468 it can be used as a trigger to initiate high data rate com2469 munication. Alternately, it can be used as a low rate, low 2470 consumption data radio [162]. Notably, the short range is not 2471 an issue for these applications [163], and the extremely low 2472 standby consumption is a major advantage. For example, a 2473 receiver sensitivity of $-40 \mathrm{dBm}$ is sufficient to receive a signal transmitted with $0 \mathrm{dBm}$ [164]. With low sensitivity demand, 2474 energy efficient WuRs can be implemented as a simple star 2475 topology with the number of nodes typically ranging from 2476 two to ten.

2477

1) Matching Prototypes: From the list of prototypes in 2478 Table VIII, there are $23 \mathrm{WuR}$ designs that match the criteria 2479 for the first scenario. All of these designs are ultra-low-power 2480 consuming, below $10 \mu \mathrm{W}$, and have node addressing capabili- 2481 ties. For the second scenario where WuRs can be used as a full 2482 data transceiver, five concepts [36], [66], [72], [73], [99] are 2483 found to be suitable. Four out of five of these are tested pro- 2484 totypes while the design concept by Jean-François et al. [73] 2485 is only in simulation. Nevertheless, all of them have data 2486 rate above $200 \mathrm{kbps}$ while exhibiting power demand below 2487 $10 \mu \mathrm{W}$.

2488

2) Suitable Protocols: From the system design perspec- 2489 tive, there are a few WuR enabled MAC protocols specif- 2490 ically designed for BAN applications. To offer high data 2491 rate and low latency, all of these are always-on wake-up 2492 MACs. The protocol proposed in [136] is transmitter-initiated 2493 while AWD-MAC [141] is receiver-initiated. However, we 2494 argue that the MAC protocols suitable for WBAN should 2495 be bi-directional so that anomaly can be reported effec- 2496 tively and on-demand. Protocols such as those presented 2497 in [42], [133], [134], and [138] are best suited for this. For 2498 communicating data, WBAN applications require either star 2499 or single-hop network, therefore, the complex routing protocol 2500 is not essential. 


\section{B. Smart City}

2503 The concept of the Smart City is growing in popularity 2504 as sensors placed throughout cities are used to support both 2505 the public administration as well as citizens directly. A large 2506 number of the placed sensors exploit wireless communication 2507 and are battery powered, allowing them to be opportunistically 2508 placed. Nevertheless, this necessitates low power operation.

2509 Today, a majority of smart city nodes communicate 2510 wirelessly over a variety of links such as IEEE802.15.4, 2511 IEEE802.15.4g, IEEE802.15.1 (Bluetooth), or low-power 2512802.11 [165]. WuRs can play a critical role in making these 2513 networks more energy-efficient, scalable, and autonomous. For 2514 example, a single-hop case can be built in which a mobile 2515 data collector, e.g., a bus or garbage truck, is equipped with a 2516 WuR. This mobile data collector traverses the city and collects 2517 information from WuR based sensing nodes deployed along 2518 its route. The sensing nodes will only be activated when the 2519 mobile data collector sends the WuS querying these nodes for 2520 data (on-demand) [17]. The feasibility of utilizing WuRs for 2521 data aggregation and for opportunistic networking in a smart 2522 city scenario has been demonstrated in [166].

2523 Infrastructure monitoring is also possible by using WuRs in 2524 a multi-hop manner [167]. A stationary or mobile data collec2525 tor can gather data from a chain of sensors attached to a bridge, 2526 tunnel or simply along the streets. WuR enables the higher 2527 power sensing nodes to remain in low energy mode when 2528 there is no data to send. Instantiating this scenario, however, 2529 necessitates a solution for the mismatch between the typical 2530 distance of the WuR and that of the primary radio.

2531 1) Matching Prototypes: In order for the WuR to be suit2532 able for smart city applications, it should support reasonable 2533 data rate, long communication range for wider coverage and 2534 low power operation. We have identified four prototypes that 2535 meet these specifications [36], [40], [54], [67], i.e., prototype 2536 numbers 15, 21, 41 and 67 listed in Table VIII. Most of the 2537 prototypes in Table VIII do not meet this criterion since either 2538 power consumption is high, a factor that limits node lifetime 2539 if battery powered or has shorter communication range than $254040 \mathrm{~m}$. Non-RF WuRs are not suitable due to the requirements 2541 imposed by the hardware such as sensitivity and LOS for 2542 optical based systems.

2543 2) Suitable Protocols: The sensors deployed within the 2544 smart city may either report periodic or on-demand data with 2545 various traffic loads. This adds an additional reliability crite2546 rion in addition to coverage and responsiveness. For instance, 2547 infrastructure monitoring systems demand fast responsiveness 2548 and should be energy efficient. That is, the events should be 2549 rapidly detected and reliably communicated in an energy effi2550 cient way through a multi-hop network for post-processing. 2551 Thus, the protocol should support event-triggered as well as 2552 periodic sensing. Various flavors of the surveyed MAC proto2553 cols can be adopted. For low latency, broadcast based MAC 2554 protocols such as PTW, AWD-MAC, and STEM-B are suit2555 able candidates. Sensors that may rely on energy harvesting 2556 technologies can utilize SLAM, WUR-TICER, and DoRa as 2557 main MAC. If a specific node is to be queried bi-directional 2558 MACs such as W-MAC are applicable. For periodic sensing where nodes can be switched off during periods of inactivity, 2559 duty-cycle wake-up MAC should be considered.

2560

After a certain duration, nodes may fail due to battery deple- 2561 tion or other external factors, therefore, new routes have to be 2562 established. Thus, the routing protocols should be adaptive and 2563 provide support for multi-hop data collection. For rapid data 2564 dissemination, network flooding protocols such as ZIPPY and 2565 FLOOD-WUP should be adopted.

2566

\section{Smart Metering}

2567

Smart meters enable remote, wireless reading of current 2568 meter values, eliminating the need for a technician to enter 2569 the home. Typical installations today place a mains powered, 2570 wireless communication unit on the meter and a mobile unit 2571 carried by a technician in a mobile vehicle. While this saves 2572 the time and energy of the technician to visit each meter, the 2573 radio itself must be powered to wait for the reading signal. 2574

Instead, a utility meter equipped with a WuRx [168] can be 2575 activated on-demand, requiring zero or near-zero consumption 2576 in between readings. To be acceptable, the solution must have 2577 ultra-low consumption (10+ years battery lifetime at 1 read- 2578 ing per month). Since utility meters are usually placed inside 2579 the building, it should also have good radio signal penetra- 2580 tion and high sensitivity operating in a sub-GHz frequency. 2581 Typically a communication distance of $15 \mathrm{~m}$ is required. 2582 According to communication standards for smart metering 2583 in Europe [169], the maximum allowed effective radiated 2584 power (ERP) in $868 \mathrm{MHz}$ band is $25 \mathrm{dBm}$. A receiver with 2585 a minimum sensitivity of $-75 \mathrm{dBm}$ will be able to receive 2586 packets at a distance of $15 \mathrm{~m}$. The required data rate for 2587 smart metering applications is moderate, supporting data rates 2588 between $2.4 \mathrm{kbps}$ and $200 \mathrm{kbps}$. Moreover, the WuR should 2589 have addressing ability in order to query specific smart meter 2590 with its unique serial number.

2591

1) Matching Prototypes: From Table VIII, eight prototypes 2592 match the requirements imposed by smart metering applica- 2593 tion. The designs presented in [36], [37], [40], [54], [56], 2594 [61], [62], and [67] exhibit power consumption below $60 \mu \mathrm{W}_{2595}$ with good receiver sensitivity and node addressing capabilities 2596 while offering tens to hundreds of kbps data rate. 2597

2) Suitable Protocols: Usually, the communication will be 2598 infrequent and demand-driven, i.e., upon a request from the 2599 data collector, therefore, polling based (taking-turns) MAC 2600 protocols best suits smart metering applications. With regard 2601 to routing, various WSN protocols may be considered [170]. 2602 However, mostly WuR-enabled meters will communicate to 2603 the collector in one-hop, then complex routing protocols are 2604 not suitable but require to maintain end-to-end reliability with 2605 nodes to be uniquely identified.

\section{Wildlife Monitoring}

2607

Use of sensor networks for wildlife monitoring has gained 2608 momentum in the recent years. Wildlife monitoring is essen- 2609 tial for keeping track of endangered wild animal movement 2610 patterns, habitat utilization, population demographics, snar- 2611 ing and poaching incidents and breakouts. For example, 2612 
2613 WildScope [171] project attaches sensor nodes on wild ani2614 mals like deer and foxes to track and to study their interaction 2615 and feeding behavior.

2616 Data collection from wildlife has been one of the hindrances 2617 in the past, thanks to sensor equipped animal collars it is much 2618 easier and cheaper now. These collars have various integrated 2619 technologies like GSM and GPS module for tracking, high 2620 power transceivers with long range for animal proximity detec2621 tion and wireless data off-loading. Due to continuous mobility, 2622 the collars require battery power with lifetime extending from 2623 few weeks to months.

2624 To prolong the lifetime, animal collars mostly use duty 2625 cycling MACs such as low power listening (LPL), where the 2626 nodes periodically wake-up, transmit the data and then go back 2627 to the sleep state. Normally, low sampling intervals ranging 2628 between an hour and a day is chosen, since a higher sampling 2629 rate would deplete batteries too quickly. However due to the 2630 periodic operation, if there are any events of interest such as 2631 interactions between animals during this inactive period of the 2632 sensor node, it will be missed and not detected at all.

2633 The problems mentioned above motivates the use of WuR 2634 technique for lifetime extension in wildlife monitoring sce2635 narios. Similar to health-care, the benefit of WuR for wildlife 2636 monitoring purposes can be two-fold: either it can be used 2637 as a "contact sensor" or as an initiator for data communica2638 tion. For example, collars designed in WildScope [171] project 2639 use high power CC2420 radio to listen to the beacon chan2640 nel for a length of time and captures the ID number of the 2641 nodes within its proximity. This method for contact detection 2642 is expensive in terms of high idle listening power consump2643 tion. Instead, WuRs can be used as a "contact sensor" while 2644 sniffing the channel for detecting other WuRs in proximity. 2645 In this manner, all the proximity beacons between animals 2646 can be captured in an energy efficient way. Not only it will 2647 reduce power consumption, but it will also reduce the latency 2648 of contact detection due to always on feature of WuR. One 2649 such example can be found in [146] where researchers have 2650 utilized WuRs to monitor contacts and encounters between 2651 individual bats.

2652 As a communication initiator, WuRs can be used to trigger 2653 nodes in a multi-hop network for offloading data to the base 2654 station, where a logical connectivity map can be constructed. 2655 Researchers can put data collectors equipped with WuR plus 2656 data transceiver and large energy supply near places where 2657 animals are expected to aggregate such as water source or 2658 ponds. When the animals are within the range of the data 2659 collector, the radio on them will be triggered by the WuR 2660 Then the collars can start transmitting the gathered sensor data 2661 via the main data transceiver to the data collector. Hence, the 2662 collars may last for years and the battery replacement and 2663 retrieval cost can be saved.

2664 1) Matching Prototypes: The salient criteria for WuRs for 2665 wildlife applications is that it should be low cost, power 2666 efficient and communication range $(>30 \mathrm{~m})$ that allows the 2667 network to cover a much larger area with few devices. The 2668 prototypes that match smart city applications are also suit2669 able here but do not demand high data rates. Nevertheless, the 2670 performance of radios in terms of communication range may degrade when moved to environments with varying vegetation, 2671 thus radios with high sensitivity plays a key role. 2672

2) Suitable Protocols: As far as MAC protocols are con- 2673 cerned, it should support both event-driven mode for appli- 2674 cations like contact sensing as well as the periodic mode for 2675 data off-loading. Thus, adaptive MAC approach is required 2676 where during inactivity, the collars can save energy by duty 2677 cycling the WuRs and during encounters with other collars it 2678 can switch to continues listening mode. The MAC protocol 2679 should be able to dynamically adapt taking into account the 2680 collar activity.

2681

In wildlife applications data is usually collected in delay- 2682 tolerant manner where it is stored locally and forwarded to the 2683 gateway when encountered with the mobile or fixed collector 2684 nodes. Low volume data can be forwarded using proactive 2685 routing algorithms that use shortest path such as EAR [152] 2686 or CTP-WUR [151].

\section{E. Security and Surveillance Systems}

Traditional security systems are based on high power cen- 2689 tral cameras that process and generate alarms if unauthorized 2690 objects or personnel are detected within the premises. Such 2691 systems are power hungry due to heavy image process- 2692 ing algorithms and require installation near the stationary 2693 power source. For applications such as continuous monitor- 2694 ing of large and wide area facilities, i.e., power plants, border 2695 lines, large factories, gas and oil pipelines with no stationary 2696 power source, infrastructure for cabling can, therefore, be very 2697 expensive.

2698

WuRs with small, low cost and low power camera systems 2699 can thus be used to detect unauthorized objects, beyond the 2700 perimeter of some critical infrastructure. The monitoring area 2701 can be covered with several WuR based camera systems, 2702 working independently and stationary. All these units will be 2703 wirelessly connected to the main system for decision making. 2704 Once an intrusion is detected via wake-up cameras, the more 2705 powerful camera system can be triggered for verification and 2706 security action. To further reduce the camera activities, low 2707 power sensors with WuRs can be added as a separate network 2708 tier. The benefits for multi-modal sensing has been proposed 2709 in [172] and its extension with WuR is presented in [15]. The 2710 authors have presented a two-tier WSN for video surveillance 2711 applications where the communication between the PIR sensor 2712 nodes and the camera nodes is performed over the wake-up 2713 receivers.

1) Matching Prototypes: The coverage and the response 2715 latency are the important criteria for this application. The 2716 WuRs should be able to react quickly based on the information 2717 provided from the sensors thus requiring high data rates. Even 2718 WuRs consuming few milliwatts are suitable as long as com- 2719 munication range is greater than $50 \mathrm{~m}$ and data requirement 2720 is satisfied. The prototype designs by Petrioli et al. [39] and 2721 Hambeck et al. [67] are the ones that fulfill these requirements. 2722

2) Suitable Protocols: Although duty cycling the WuRs 2723 on camera nodes will reduce power consumption, it also 2724 introduces response latency. To keep the latency at bay, an 2725 alternative solution is to use MAC protocols that are based on 2726 
2727 always on WuRs and continuously monitor the channel while 2728 keeping power consumption low (e.g., W-MAC). With regard 2729 to routing, a cost effective and reliable multi-hop communica2730 tion network that relays the monitored information in a timely 2731 manner is required so that efficient monitoring of the area can 2732 take place.

\section{F. Indoor Localization}

2734 In the recent past, robust and accurate indoor localization 2735 for navigating has become one of the challenging areas for the ${ }_{2736}$ WSN community since the GPS does not work indoors. One 2737 of the demanding applications of indoor localization besides 2738 navigation in shopping malls, user or robot localization, and 2739 environment modeling, is support for rescue teams during 2740 emergency scenarios. In life-threatening situations such as fire, 2741 rescue teams can often lose their orientation in smoky areas 2742 due to low visibility.

2743 To increase the indoor localization accuracy within millime2744 ters, these systems employ external reference points known 2745 as landmarks, for instance, $\mathrm{Wi}-\mathrm{Fi}$ access points or ultra-wide 2746 band systems for taking extra measurements like Received 2747 Signal Strength Indicator (RSSI) or the Time Difference of 2748 Arrival (TDoA). These landmarks consume high energy, and 2749 either they require a continuous power supply or the bat2750 teries have to be changed frequently if always kept on. In 2751 catastrophic scenarios when there is no power available from 2752 the grid or if the batteries run out, landmarks will be of 2753 no use.

2754 Integrating wake-up technology into these landmarks has 2755 the potential to extend the lifetime with improved energy con2756 sumption. Simon et al. [173] presented the idea of developing 2757 new WuR enabled wireless landmarks such as smoke detec2758 tors. During inactivity, these landmarks can be put into sleep 2759 state to reduce unnecessary energy wastage.

$2760 \quad 1)$ Matching Prototypes: The requirements for WuRs in the 2761 localization case are low power consumption, a communica2762 tion range of few meters, and data rate in the region of several 2763 hundred kb/s. Moreover, there will be many landmarks within 2764 a building with devices operating at the similar frequency, 2765 therefore, the WuR should provide improved resistance to 2766 interference to prevent false wake-ups. WuRs operating in $2767 \mathrm{sub}-\mathrm{GHz}$ with communication range above $10 \mathrm{~m}$ should be 2768 preferred.

2769 2) Suitable Protocols: In indoor localization applications, 2770 the navigating node will be frequently requesting the data 2771 from the anchor or landmarks deployed within the vicinity 2772 for updating the localization information. Thus, always on 2773 WuR-enabled MAC protocols are best suited for this. However, 2774 bi-directional communication is a must as the information will 2775 be shared to and from these landmarks. For emergency appli2776 cations, the key requirement is to deliver messages in real-time 2777 and with a high probability of success, a challenging task in 2778 wireless sensor networks. To satisfy this requirement, adap2779 tive or opportunistic routing protocols should be adopted to 2780 avoid routing holes (caused by nodes that have failed) or seek 2781 real-time and valid paths in emergency situations.

\section{G. Asset Tracking}

To improve operational efficiency in commercial businesses 2783 and to deliver quality customer experience, asset tracking 2784 during various phases is essential. Businesses as well as cus- 2785 tomers, both want to identify, locate and manage their assets 2786 in a timely manner. Traditionally, this process was done man- 2787 ually by registering product IDs when the items pass through 2788 certain warehouses or locations. A slightly faster method was 2789 introduced by use of bar codes for tracking items. However, 2790 these methods are time consuming and prone to human error. 2791 Recently, RFID technology based solutions have become more 2792 preferred choice of tracking items that uses radio signals. The 2793 items are attached with passive RFID tags and an active RFID 2794 reader is used to send signals to acquire data from these tags. 2795 Due to passive nature of the tags, the communication range is 2796 usually limited up to a few centimeters and to achieve up to 2797 few meters, large antennas are required.

2798

To ameliorate above mentioned issues, active RFID tags 2799 have been integrated with wireless sensor nodes [174] such 2800 that the integrated tags are able to communicate with many 2801 wireless devices which are not limited to readers. The RFID 2802 system provides the product IDs while other information is 2803 communicated using the main node's radio. Consequently, 2804 active RFIDs are too costly and power hungry. Therefore, 2805 to bridge the gap between RFID and WSNs, RFIDs can be 2806 replaced with WuRs. For example, the WuRs can periodically 2807 transmit radio beacons that may contain the product ID and 2808 the timestamps forming an "smart object". Moreover, using 2809 the built-in selective wake-up method, these beacons can also 2810 serve as object selector. Thus, allowing specific nodes to be 2811 queried on demand.

2812

Malinowski et al. [119] presented the idea of quasi-passive 2813 wakeup for asset monitoring. In this work WuRx has been 2814 integrated with sensor nodes acting as tags. Whenever the 2815 base station queries the tags for events, the wake-up receivers 2816 compare the signals against a threshold before activating the 2817 main CC2500 radio. If there are no queries, the main radio 2818 goes into sleep mode and the WuRx is kept active consuming 2819 only $25 \mu \mathrm{W}$ of power. Another specialized tag embedded with 2820 wake-up radios and sensors has been recently developed [57] 2821 for indoor and outdoor asset tracking. The design is extremely 2822 power efficient, low cost and supports dual frequency for 2823 communication.

2824

1) Matching Prototypes: To realize wake-up radio based 2825 enhanced smart objects long-term operation is an essential 2826 requirement. Energy harvesting WuRs such as those proposed 2827 in [93], [95], and [99] are suitable alternatives for enabling 2828 autonomous long-term operation with minimum maintenance 2829 cost.

2830

2) Suitable Protocols: There are two types of nodes utilized 2831 in asset tracking; the gateway that is connected to the on-line 2832 database and the reader nodes associated with each type of 2833 items. To successfully locate these objects, the bi-directional 2834 communication mechanism is essential where the gateway 2835 can query the reader nodes by requesting information while 2836 the reader nodes can respond through their WuRs. On the 2837 data collection side, energy-efficient and low-power routing 2838 
2839 protocol is needed for continuous asset tracking applications. 2840 Moreover, in storage facilities such as warehouses where hun2841 dreds of sensor tags equipped with WuRs might be present, 2842 packet losses and interference will be an issue. Therefore, 2843 robust algorithms to counteract this issue needs to be consid2844 ered. One possible solution is to use multi-channel protocols 2845 with the node-addressing feature.

\section{${ }_{2846}$ H. Wearables}

2847 Nowadays, wearable electronics have the huge potential to 2848 enhance people's lives every day. New devices like activity 2849 trackers, smart bracelet, smart clothes have appeared in myr2850 iad, bundled with appealing Apps and motivating people to 2851 be always looking forward to new services. Similar to most 2852 of the battery operated devices (e.g., smartphones), wearable 2853 electronics tackles the need to prolong the battery autonomy 2854 as long as possible as well as keeping the size small for 2855 comfortable wearing. The challenge is even harder if con2856 sidering that most of the tasks required by wearable devices 2857 are data-streaming oriented (e.g., headphones, trackers, fitness 2858 equipment) and energy efficiency is a key for such devices.

2859 The presence of WuR methods would enhance the device 2860 reducing remarkably the energy spent in idle time, when the 2861 user is not ready or not connected to the specific device, or 2862 not requesting for a specific service. Strategies, where wear2863 able devices are combined with ultra-low power wake up 2864 radio have been already presented in [175]. Moreover, con2865 text aware applications can decide which wearable object need 2866 to be activated avoiding overlapping of services when not 2867 needed. Typically, wearable objects are connected using a well 2868 known and widespread wireless standard (e.g., Bluetooth Low 2869 Energy) to a smartphone, that is used as a central device 2870 for processing and forwarding the information to the Internet. 2871 Considering that nowadays, smartphones follow owners almost 2872 all the day, the communication range of the WuR is not 2873 an issue and very low standby power consumption can be 2874 achieved.

2875 1) Matching Prototypes: Wearable electronics share some 2876 characteristics typical to the WBANs, and considering the 2877 short distance, potentially several WuR designs reviewed in 2878 this survey could satisfy the application requirements, such 2879 as [36], [66], [72], [73], and [99]. Nonetheless, most of 2880 the wearable devices offer BLE connectivity and some are 2881 equipped with Low-Power Wi-Fi. A WuR technology design 2882 in the $2.4 \mathrm{GHz}$ such as one in [96] could facilitate in future 2883 the transition towards a comprehensive radio-on-chip which 2884 includes a wireless standard and WuR technology.

2885 2) Suitable Protocols: There are a few WuR enabled MAC 2886 protocols specifically designed for wearables applications, and 2887 to the author's knowledge none are specifically integrated 2888 into a standard like BLE or low-power Wi-Fi. To offer high 2889 data rate and low latency, a MAC could intensively be called 2890 by the wake-up event. Protocols such as those presented 2891 in [42] and [133] are suitable for the wearable scenario and 2892 offer insights for an integration in Bluetooth radio protocols.

\section{I. Smart Grid}

2894 Microgrids is a new trend for achieving energy efficiency in 2895 the distribution of the electrical energy. It is revolutionizing the normal electrical grids within the Smart grids. Realtime con- 2896 trol services for monitoring the quality of the power distributed 2897 from big power generation plants toward small and distributed 2898 network make information and communication technology 2899 more crucial than in the past.

2900

One of the main challenges of the smart grid applications 2901 is relying on efficient communication infrastructure and ser- 2902 vice. Communication between measurement points is often 2903 realized using heterogeneous technology, both wireless and 2904 wired. Among these, power line communications (PLC) is a 2905 straightforward non-wireless choice. Several wake-up mecha- 2906 nisms that share similar medium, requirements, and protocols 2907 have been already proposed [176], [177].

2908

A wake-up based approach can be implemented with a 2909 very simple and low-power device that constantly observes 2910 the communication channel and informs a host system when- 2911 ever activity is detected. Since the power consumption of the 2912 wake-up is lower than PLC receiver, the overall energy con- 2913 sumption of the communication in the microgrid is drastically 2914 reduced.

2915

1) Matching Prototypes: Micro-grid communication often 2916 includes heterogeneous technologies. Some of the prototypes 2917 that suit smart grid requirements are presented in [36], [67], 2918 and [178], while non-RF wakeup circuits such as those 2919 in [176] and [177] also exists even if with features tailored 2920 for cable communication [177].

2921

2) Suitable Protocols: Protocols for wireless communica- 2922 tion in smart grids may either report periodic or on-demand 2923 data with various traffic loads. This requires also fast respon- 2924 siveness at the lowest energy cost. MAC protocols, therefore, 2925 should focus on the latency optimization and on the multi-hop 2926 characteristic of the network. In these cases, protocols such 2927 as PTW, AWD-MAC, and STEM-B are suitable candidates. 2928 For the wake-up mechanism developed over PLC (on cables), 2929 the protocol needs tight synchronization and the one proposed 2930 in [176] is suitable for the purpose.

\section{J. Discussion}

2932

This is certainly not an exhaustive list, with are many other 2933 applications that can benefit from WuR technology includ- 2934 ing building automation, smart lighting, remote keyless entry, 2935 aerospace to name a few.

2936

Depending on application demands, the requirements for 2937 low power WuRs differ. For some applications, a high data 2938 rate is essential, while for others long communication range 2939 is of importance.

2940

Table XII provides an overview of different application 2941 requirements, which can be used as a reference for system 2942 developers to assist in categorizing and choosing the appro- 2943 priate low power WuR. However, one should note that these 2944 requirements are not meant to be strict, but rather offer guide- 2945 lines that one should keep in mind while designing WuR based 2946 systems.

2947

As can be seen, the health-care case has the highest 2948 demand for data rate because of possible multimedia appli- 2949 cations and most stringent power requirements. The highest 2950 requirement for communication range is given in the smart 2951 city application case, closely followed by wildlife monitor- 2952 ing applications. Only health care applications have moderate 2953 
TABLE XIII

Summary of Issues, Challenges, AND Opportunities For WAKe-Up Radio Based Hardware and Software Designs

\begin{tabular}{|c|c|c|c|}
\hline Category & Dimensions & Issues / Challenges & Opportunities \\
\hline \multirow{3}{*}{$\begin{array}{l}\text { Hardware } \\
\text { Design }\end{array}$} & $\begin{array}{l}\text { Cost and Technology } \\
\text { Integration }\end{array}$ & $\begin{array}{l}\text {-short communication range } \\
\text {-high deployment cost } \\
\text {-separate radio modules }\end{array}$ & $\begin{array}{l}\text {-small form factor designs } \\
\text {-cheaper SoC } \\
\text {-single chip packaging }\end{array}$ \\
\hline & Power Demand & $\begin{array}{l}\text {-always on receivers } \\
\text {-low receiver sensitivity } \\
\text {-non-negligible listening power } \\
\text {-reduced data rate } \\
\text {-high transmission power }\end{array}$ & $\begin{array}{l}\text {-design of energy harvesting WuRs with low latency } \\
\text {-ultra low power transmitter designs } \\
\text {-novel hardware design with short and } \\
\text { long range capabilities } \\
\text {-design of low power, high sensitivity WuRs }\end{array}$ \\
\hline & System Architecture & $\begin{array}{l}\text {-no unified system and } \\
\text { networking architecture }\end{array}$ & $\begin{array}{l}\text {-WuR designs with multiple integrated sensors } \\
\text {-modular architecture for easy integration } \\
\text {-flexible and open source designs }\end{array}$ \\
\hline \multirow{6}{*}{$\begin{array}{c}\text { Software } \\
\text { [Protocol Designs] }\end{array}$} & Channel Sharing & $\begin{array}{l}\text {-static channel assignment } \\
\text {-asymmetric network thus mismatch } \\
\text { of transmisson ranges }\end{array}$ & $\begin{array}{l}\text {-multichannel MAC and routing protocols } \\
\text {-dynamic spectrum selection } \\
\text {-dynamic channel handover } \\
\text {-WuR integration with cognitive radios }\end{array}$ \\
\hline & $\begin{array}{l}\text { Synchronous } \\
\text { WuR-MAC }\end{array}$ & -time synchronization & $\begin{array}{l}\text {-synchronous transmission over WuR } \\
\text {-efficient time synchronization mechanism } \\
\text { with low overhead }\end{array}$ \\
\hline & Adaptive Protocols & $\begin{array}{l}\text {-static network parameters } \\
\text {-non-adaptive }\end{array}$ & $\begin{array}{l}\text {-design of traffic adaptive protocols } \\
\text {-dynamic route maintenance }\end{array}$ \\
\hline & Mobility & $\begin{array}{l}\text {-static nodes } \\
\text {-unstable mobile routes }\end{array}$ & $\begin{array}{l}\text {-design of mobility based routing } \\
\text {-need for novel topology aware routing } \\
\text { with minimum overhead }\end{array}$ \\
\hline & $\begin{array}{l}\text { Interference and } \\
\text { Coexistence }\end{array}$ & $\begin{array}{l}\text {-high interference with nearby } \\
\text { in-band devices }\end{array}$ & $\begin{array}{l}\text {-multichannel MAC and routing } \\
\text {-dynamic channel hand-over mechanisms } \\
\text {-robust wake-up beacon modulation techniques }\end{array}$ \\
\hline & Standardization & -none available & $\begin{array}{l}\text { Requires standardization of: } \\
\text {-frequency } \\
\text {-channel availability } \\
\text {-wake-up beacon format } \\
\text {-hardware design }\end{array}$ \\
\hline
\end{tabular}

2954 sensitivity requirements owing to the shorter communication 2955 range.

2956 Generally, all applications demand node addressing capabil2957 ity in order to query particular nodes.

2958 Further applications can be realized if wake-up radios 2959 are designed with standalone devices. An integration 2960 into transceivers as a substitution for built-in wake-on2961 radio mechanisms can further optimize these applications. 2962 Finally, low power consumption for WuRs in the ultra-high 2963 frequency (UHF) band offers a vast number of new services 2964 and applications.

\section{IX. Open Issues, Challenges AND $2966 \quad$ FUTURE RESEARCH DIRECTIONS}

2967 This section presents some of the main issues and chal2968 lenges that must be addressed while designing systems based 2969 on WuRs. The challenges are not only related to hardware 2970 designs but also to the design and efficiency of upper layers 2971 of the stack. We then discuss some of the research directions 2972 that can be taken to mitigate these issues as discussed next 2973 and presented in Table XIII.

\section{A. Hardware Design}

2975 The evolution of the WuR technology is mainly driven by 2976 advancements in core technology and the demand for ever-less 2977 power consumption.

2978 1) Cost and Technology Integration: Cost is one of the 2979 major factors, which is taken into consideration when design2980 ing and deploying large scale WSNs. So far, the small form factor and low hardware cost have been the key success indi- 2981 cator for WSNs. With the inclusion of WuR, the overall 2982 cost is expected to rise and can become one of the hur- 2983 dles of this method. Further, the cost of designing ultra-low 2984 power WuR is still challenging. Current WuR have a shorter 2985 communication range than the traditional radios, making it 2986 difficult to align coverage of these two radios. For wide area 2987 coverage, high-density deployment will be required leading 2988 to higher maintenance costs. Recently, to address this issue 2989 Magno et al. [179] have proposed a new IoT node integrated 2990 with LoRa technology and energy harvesting wake-up receiver 2991 for long and short range networking. Another design that fuses 2992 wake-up radio and BLE technology with energy harvesting has 2993 appeared in [96].

2994

Most of the presented features, such as addressing and 2995 in- or out-of-band communication, need to be implemented 2996 in a single chip with the main radio. Keeping a dual radio 2997 mechanism using separate components is expensive for IoT 2998 device production. This also includes the RF front-end circuits 2999 whose WuR performance mostly depends on the chip design. 3000 The possibility to have everything pre-assembled or packaged 3001 in a well-characterized module or component will pave the 3002 way to create a mass diffusion of such technology. An inte- $з 003$ grated design including the non-volatile baseband processor 3004 with wake-up identification receiver and power management 3005 module has been recently proposed in [118]. Although the 3006 architecture has been tested only using simulations, it opens 3007 up new hardware design opportunities.

3008

2) Power Demand: In WuR based systems, always-on 3009 WuRs constantly dissipate energy, thus designing a transceiver 3010 
3011 that consumes orders of magnitude less than the main radio is 3012 necessary. The power demand of WuRs is also dependent on 3013 other factors such as reception sensitivity and data rate, which 3014 dictates the radios performance. All these factors must be 3015 considered and the trade-offs among them should be exploited. 3016 While passive wake-up radios are an attractive and alter3017 native means to save energy, it also poses few challenges. 3018 Harvested energy is very sensitive to environmental conditions 3019 and where energy sources are not always available, the wake3020 up procedure may be delayed. For delay-sensitive applications, 3021 such designs may not be suitable. Therefore, an open issue 3022 is how to reduce this delay with passive systems. Recently 3023 Mahapatra et al. [180] have investigated how to use energy 3024 harvesting based wake-up radios together with error control 3025 coding to enhance the performance of networks while reducing 3026 carbon footprint.

3027 Further, passive WuRs have shorter communication ranges 3028 than active ones. The wake-up signals are transmitted at high 3029 power to achieve long range thus incurring high energy cost. 3030 This demands low power wake-up transmitter designs similar 3031 to wake-up receivers that are simple to implement, turn ON 3032 almost instantly, transmit a short WuS and go back to the sleep 3033 state. A few works have proposed techniques such as the use 3034 of directional antennas [35], antenna diversity [34], and ultra 3035 long range RFID [101] to improve the transmission range of 3036 these radios.

3037 At the same time, power consumption and receiver sensi3038 tivity will still be the major drivers to determine the future зозя direction of WuRs; because they characterize the operating 3040 range of $\mathrm{WuR}$. The transmission range of any radio commu3041 nication will be the major driver for the coming generation of 3042 IoT devices. Low power communication is rapidly evolving 3043 towards multi-kilometer ranges and low bit-rate schemes. Long 3044 range sub-GHz radios such as LoRa [181] or Sigfox [182] are 3045 pioneers of this IoT communication revolution. If WuR tech3046 nology does not advance with its features, it will be hampered 3047 in this market.

3048 3) System Architecture: Currently no unified system and 3049 networking architecture exists for WuRs to build applications 3050 on top. The integration of different types of sensors, energy 3051 harvesters, and RFID tags may necessitate new and modular 3052 WuR architectures.

\section{B. Protocol Design}

3054 Although the notion of wake-up radio eliminates the com3055 plexity that is involved with duty cycling MACs, there are 3056 many other challenges that need to be taken into account. 3057 Power consumption is also affected by the channel conditions, 3058 topology of the network, and the routing protocols utilized. 3059 Some of these challenges and issues are discussed next.

3060 1) Channel Sharing: Sharing channels between wake-up 3061 and main radios must be studied since these two network lay3062 ers have mismatched transmission ranges, forming an asym3063 metric network. Designing protocols that are more responsive 3064 to channel changes is still an open issue. There are a few 3065 research works that have attempted to address this such as 3066 CTP-WUR [151], Guo et al. [125], and WUR-MAC [130].
One solution to opportunistic spectrum access is using cogni- ${ }_{3067}$ tive radios. Recently, cognitive radios have been incorporated 3068 in sensor networks [183]-[185]. Traditional radios assume 3069 fixed channel allocation and usually operate in crowded unli- 3070 censed bands that are also used by other devices making them 3071 prone to interference and collisions. Cognitive radios have the 3072 ability to opportunistically select the unused spectrum either 3073 in a licensed or unlicensed band. Combining WuRs with cog- 3074 nitive radio may enhance the overall system performance by 3075 increasing the communication reliability, alleviating collisions 3076 and packet losses, and improving the energy efficiency in 3077 dense networks. Due to its dynamic spectrum selection mech- 3078 anism, multiple overlaid networks can also be realized without 3079 channel contention.

3080

A few works have proposed dynamic channel selection by 3081 integrating wake-up radio with Wi-Fi modules. Specifically, 3082 Yoshiwaka et al. [186] utilized a frame length detection mech- 3083 anism with OOK modulation for selecting the appropriate 3084 Wi-Fi channel for transmission. Instead of only using wake-up 3085 radios for remote triggering, Tang et al. [187], [188] have also 3086 used it for carrier sensing before transmission by integrating it 3087 with WLAN. Standardization of wake-up receiver integration 3088 with WLAN has also started [189].

3089

2) Synchronous WuR-MAC: Wake-up radios can also be 3090 utilized with synchronous MAC protocols for reducing latency 3091 and energy consumption [37]. However, such designs require 3092 time synchronization among the nodes. WuRs are even more 3093 resource constrained devices than typical motes in terms of 3094 processing power, memory, available energy, and communica- 3095 tion bandwidth. Thus, complex time synchronization protocols 3096 and heavy control overheads may not be feasible and requires 3097 careful design.

3098

3) Adaptive Protocols: As seen in Section VIII, WuRs can 3099 be utilized for applications that have harsh environments such 3100 as structural, animal monitoring or for emergency response 3101 where nodes are prone to failures. This may lead to other 3102 issues such as transmission failure or long latencies due to 3103 poorly designed MAC and routing protocols. To mitigate this, 3104 robust and adaptive protocols utilizing WuRs needs to be 3105 designed. These protocols should be traffic adaptive, avoid 3106 routing holes, and establish new routes dynamically in order to 3107 deliver messages reliably and in real-time. WuRs also exhibit 3108 shorter communication range than main radios. The design of 3109 such protocols is an open research direction.

3110

4) Mobility: Another possible area of research is the 3111 consideration of node mobility in wakeup schedule design 3112 (e.g., [145] and [146]). Most of the existing schemes assume 3113 that the sensor nodes and sink are stationary. Asynchronous 3114 and non-collaborative synchronous schemes are good candi- 3115 dates for these scenarios because their lack of coordination 3116 requirement makes them robust to network topology changes. 3117 In the presence of node mobility, schemes that require coor- 3118 dination may not converge to an optimal schedule or may 3119 generate excessive overhead. How WuRs will behave in such 3120 situations is still unknown.

3121

5) Interference and Coexistence: The propagation impair- 3122 ments of wake-up radio signals in harsh environments such 3123 as forest, industrial or inside human-body also needs to be 3124 
3125 considered while designing WuR based systems. According 3126 to our survey, this so far has not been widely studied. An 3127 initial study by Lebreton et al. [190] looks into the in-band 3128 interference from nearby Wi-Fi devices on a wake-up radio 3129 system. The results indicate that wake-up radios are able 3130 to maintain high performance in coexistence with external 3131 wireless networks while slightly compromising on energy effi3132 ciency. Further investigation and study of the aforementioned 3133 propagation issues in different settings need to be conducted. 3134 6) Standardization: It is important to remark that there is 3135 a clear lack of standardization activities related to the WuR 3136 designs such as (i) frequency usage, (ii) available channels, 3137 (iii) maximum power below which a radio can be classified as 3138 a WuR, (iv) wake-up signal format, and (v) routing topology. 3139 To address this, in July 2016, a wake-up radio study group 3140 (WUR SG) has been set up within the IEEE 802.11 working 3141 group to standardize the above activities [189]. The main aim 3142 of this group is to enable an energy efficient data reception 3143 for wake-up radios integrated with WLANs without increase 3144 of latency. An attempt has also been made to standardize the 3145 wake-up radio packet structure so that it is compatible with 3146 different technologies in the area of medical applications [41].

\section{COnClusion}

3148 Our survey identifies growing interest across the many 3149 facets of the design space of wake-up radios. Available hard3150 ware is expanding, with improvements in range, sensitivity 3151 and consumption. Protocol stacks are emerging to exploit the 3152 novel properties of this technology, opening new application 3153 domains. Future work will require coordinated efforts at all 3154 levels to address limitations such as the difference in transmis3155 sion range between a wake-up receiver and a traditional, higher 3156 power receiver. Further, issues such as interference must be 3157 studied to understand the reliability and robustness of systems 3158 incorporating wake-up receivers. Nevertheless, the potential of 3159 wake-up receivers to dramatically reduce the power consump3160 tion footprint of wireless, battery powered networks has been 3161 clearly demonstrated, offering motivation for future work.

\section{REFERENCES}

[1] A. Al-Fuqaha, M. Guizani, M. Mohammadi, M. Aledhari, and M. Ayyash, "Internet of Things: A survey on enabling technologies, protocols, and applications," IEEE Commun. Surveys Tuts., vol. 17, no. 4, pp. 2347-2376, 4th Quart., 2015.

[2] B. Rashid and M. H. Rehmani, "Applications of wireless sensor networks for urban areas: A survey," J. Netw. Comput. Appl., vol. 60, pp. 192-219, Jan. 2016.

[3] I. F. Akyildiz, W. Su, Y. Sankarasubramaniam, and E. Cayirci, "A survey on sensor networks," IEEE Commun. Mag., vol. 40, no. 8, pp. 102-114, Aug. 2002.

[4] J. Suh and M. Horton, "Powering sensor networks," IEEE Potentials, vol. 23, no. 3, pp. 35-38, Aug./Sep. 2004.

[5] R. C. Carrano, D. Passos, L. C. S. Magalhaes, and C. V. N. Albuquerque, "Survey and taxonomy of duty cycling mechanisms in wireless sensor networks," IEEE Commun. Surveys Tuts., vol. 16, no. 1, pp. 181-194, 1st Quart., 2014.

[6] K. W. Choi, L. Ginting, P. A. Rosyady, A. A. Aziz, and D. I. Kim, "Wireless-powered sensor networks: How to realize," IEEE Trans. Wireless Commun., vol. 16, no. 1, pp. 221-234, Jan. 2017.

[7] D. Niyato, E. Hossain, and A. Fallahi, "Sleep and wakeup strategies in solar-powered wireless sensor/mesh networks: Performance analysis and optimization," IEEE Trans. Mobile Comput., vol. 6, no. 2, pp. 221-236, Feb. 2007
[8] Tmote Sky: Ultra Low Power IEEE 802.15. 4 Compliant Wireless 3186 Sensor Module, Moteiv Corporat., 2006.

[9] The MAC Aphabet Soup Served in Wireless Sensor Networks. [Online]. 3188 Available: http://www.st.ewi.tudelft.nl/ koen/MACsoup/ 3189

[10] J. Blanckenstein, J. Klaue, and H. Karl, "A survey of low-power 3190 transceivers and their applications," IEEE Circuits Syst. Mag., vol. 15, 3191 no. 3, pp. 6-17, 3rd Quart., 2015.

3192

[11] V. Jelicic, M. Magno, D. Brunelli, V. Bilas, and L. Benini, "Analytic 3193 comparison of wake-up receivers for WSNs and benefits over the wake- 3194 on radio scheme," in Proc. 7th ACM Workshop Perform. Monitor. Meas. 3195 Heterogeneous Wireless Wired Netw. (PM2HW2N), 2012, pp. 99-106. 3196

[12] T. Soyata, L. Copeland, and W. Heinzelman, "RF energy harvesting 3197 for embedded systems: A survey of tradeoffs and methodology," IEEE 3198 Circuits Syst. Mag., vol. 16, no. 1, pp. 22-57, Feb. 2016.

3199

13] P. Kamalinejad et al., "Wireless energy harvesting for the Internet of 3200 Things," IEEE Commun. Mag., vol. 53, no. 6, pp. 102-108, Jun. 2015. 3201

[14] R. Pozza, M. Nati, S. Georgoulas, K. Moessner, and A. Gluhak, 3202 "Neighbor discovery for opportunistic networking in Internet of Things 3203 scenarios: A survey," IEEE Access, vol. 3, pp. 1101-1131, 2015.

[15] V. Jelicic, M. Magno, D. Brunelli, V. Bilas, and L. Benini, "Benefits 3205 of wake-up radio in energy-efficient multimodal surveillance wire- 3206 less sensor network," IEEE Sensors J., vol. 14, no. 9, pp. 3210-3220, 3207 Sep. 2014

[16] H. Ba, I. Demirkol, and W. Heinzelman, "Feasibility and benefits of 3209 passive RFID wake-up radios for wireless sensor networks," in Proc. 3210 IEEE Glob. Telecommun. Conf. (GLOBECOM), Miami, FL, USA, 3211 Dec. 2010, pp. 1-5.

[17] J. Oller et al., "Has time come to switch from duty-cycled MAC proto- 3213 cols to wake-up radio for wireless sensor networks?" IEEE/ACM Trans. 3214 Netw., vol. 24, no. 2, pp. 674-687, Apr. 2016.

3215

[18] J. Oller et al., "Wake-up radio as an energy-efficient alternative to 3216 conventional wireless sensor networks MAC protocols," in Proc. 16th 3217 ACM Int. Conf. Model. Anal. Simulat. Wireless Mobile Syst. (MSWiM), 3218 Barcelona, Spain, 2013, pp. 173-180.

[19] P. Huang, L. Xiao, S. Soltani, M. W. Mutka, and N. Xi, "The evolu- 3220 tion of MAC protocols in wireless sensor networks: A survey," IEEE 3221 Commun. Surveys Tuts., vol. 15, no. 1, pp. 101-120, 1st Quart., 2013. 3222

[20] K. Han, J. Luo, Y. Liu, and A. V. Vasilakos, "Algorithm design for data 3223 communications in duty-cycled wireless sensor networks: A survey," 3224 IEEE Commun. Mag., vol. 51, no. 7, pp. 107-113, Jul. 2013.

[21] A. Bachir, M. Dohler, T. Watteyne, and K. K. Leung, "MAC essentials 3226 for wireless sensor networks," IEEE Commun. Surveys Tuts., vol. 12, 3227 no. 2, pp. 222-248, 2nd Quart., 2010.

[22] X. Fafoutis, A. D. Mauro, M. D. Vithanage, and N. Dragoni, 3229 "Receiver-initiated medium access control protocols for wireless sensor 3230 networks," Comput. Netw., vol. 76, pp. 55-74, Jan. 2015.

[23] G. Anastasi, M. Conti, M. D. Francesco, and A. Passarella, "Energy 3232 conservation in wireless sensor networks: A survey," Ad Hoc Netw., 3233 vol. 7, no. 3, pp. 537-568, 2009.

3234

[24] I. Demirkol, C. Ersoy, and E. Onur, "Wake-up receivers for wireless 3235 sensor networks: Benefits and challenges," IEEE Wireless Commun., 3236 vol. 16, no. 4, pp. 88-96, Aug. 2009.

[25] J. Hao, B. Zhang, and H. T. Mouftah, "Routing protocols for duty 3238 cycled wireless sensor networks: A survey," IEEE Commun. Mag., 3239 vol. 50, no. 12 , pp. 116-123, Dec. 2012

[26] J. N. Al-Karaki and A. E. Kamal, "Routing techniques in wireless 3241 sensor networks: A survey," IEEE Wireless Commun., vol. 11, no. 6, 3242 pp. 6-28, Dec. 2004.

3243

[27] N. A. Pantazis, S. A. Nikolidakis, and D. D. Vergados, "Energy- 3244 efficient routing protocols in wireless sensor networks: A survey," IEEE 3245 Commun. Surveys Tuts., vol. 15, no. 2, pp. 551-591, 2nd Quart., 2013. 3246

[28] M. Radi, B. Dezfouli, K. A. Bakar, and M. Lee, "Multipath routing 3247 in wireless sensor networks: Survey and research challenges," Sensors, 3248 vol. 12, no. 1, pp. 650-685, 2012.

[29] L. D. P. Mendes and J. J. P. C. Rodrigues, "A survey on cross-layer 3250 solutions for wireless sensor networks," J. Netw. Comput. Appl., vol. 34, 3251 no. 2, pp. 523-534, 2011.

3252

[30] B. Fu, Y. Xiao, H. J. Deng, and H. Zeng, "A survey of cross-layer 3253 designs in wireless networks," IEEE Commun. Surveys Tuts., vol. 16, 3254 no. 1, pp. 110-126, 1st Quart., 2014.

[31] P. P. Mercier and A. P. Chandrakasan, Ultra-Low-Power Short-Range 3256 Radios. Cham, Switzerland: Springer, 2015.

[32] P. Le-Huy and S. Roy, "Low-power wake-up radio for wireless sensor 3258 networks," Mobile Netw. Appl., vol. 15, no. 2, pp. 226-236, 2010. 3259

[33] B. V. der Doorn, W. Kavelaars, and K. Langendoen, "A prototype low- 3260 cost wakeup radio for the $868 \mathrm{MHz}$ band," Int. J. Sensor Netw., vol. 5, 3261 no. 1, pp. 22-32, 2009.

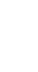

\section{AQ4}


[34] T. Kumberg, R. Tannhaeuser, and L. M. Reindl, "Using antenna diversity to improve wake-up range and probability," in Proc. Progr Electromagn. Res. Symp., vol. 2091. 2015, pp. 2779-2783.

[35] W.-C. Shih, R. Jurdak, D. Abbott, P. H. Chou, and W.-T. Chen, "A long-range directional wake-up radio for wireless mobile networks," J. Sensor Actuator Netw., vol. 4, no. 3, pp. 189-207, 2015.

[36] G. U. Gamm, M. Sippel, M. Kostic, and L. M. Reindl, "Low power wake-up receiver for wireless sensor nodes," in Proc. 6th Int. Conf. Intell. Sensors Sensor Netw. Inf. Process. (ISSNIP), Brisbane, QLD, Australia, Dec. 2010, pp. 121-126.

[37] F. Sutton, B. Buchli, J. Beutel, and L. Thiele, "Zippy: On-demand network flooding," in Proc. 13th ACM Conf. Embedded Netw. Sensor Syst. (SenSys), Seoul, South Korea, 2015, pp. 45-58.

[38] J. Ansari, D. Pankin, and P. Mähönen, "Radio-triggered wake-ups with addressing capabilities for extremely low power sensor network applications," Int. J. Wireless Inf. Netw., vol. 16, no. 3, pp. 118-130, 2009.

[39] C. Petrioli, D. Spenza, P. Tommasino, and A. Trifiletti, "A novel wake-up receiver with addressing capability for wireless sensor nodes," in Proc. IEEE Int. Conf. Distrib. Comput. Sensor Syst. (DCOSS), Marina Del Rey, CA, USA, 2014, pp. 18-25.

[40] M. Magno et al., "Design, implementation, and performance evaluation of a flexible low-latency nanowatt wake-up radio receiver," IEEE Trans. Ind. Informat., vol. 12, no. 2, pp. 633-644, Apr. 2016.

[41] C. S. Hong, M. A. Ameen, and S. I. Moon, "Emergency communication for low energy body-centric wearable networks," Internet Engineering Task Force, Internet-Draft, Apr. 2016. [Online]. Available: https://tools.ietf.org/html/draft-hongcs-6lo-bcwc-00

[42] S. Marinkovic and E. Popovici, "Ultra low power signal oriented approach for wireless health monitoring," Sensors, vol. 12, no. 6, pp. 7917-7937, 2012.

[43] J. Blobel, J. Krasemann, and F. Dressler, "An architecture for senderbased addressing for selective sensor network wake-up receivers," in Proc. IEEE 17th Int. Symp. World Wireless Mobile Multimedia Netw. (WoWMoM), Coimbra, Portugal, Jun. 2016, pp. 1-7.

[44] K. Yadav, I. Kymissis, and P. R. Kinget, "A 4.4- $\mu \mathrm{W}$ wake-up receiver using ultrasound data," IEEE J. Solid-State Circuits, vol. 48, no. 3, pp. 649-660, Mar. 2013.

[45] E. Lattanzi, M. Dromedari, V. Freschi, and A. Bogliolo, "A sub- $\mu$ A ultrasonic wake-up trigger with addressing capability for wireless sensor nodes," ISRN Sensor Netw., vol. 2013, Aug. 2013, Art. no. 720817.

[46] F. Hoflinger, G. U. Gamm, J. Albesa, and L. M. Reindl, "Smartphone remote control for home automation applications based on acoustic wake-up receivers," in Proc. IEEE Int. Instrum. Meas. Technol. Conf. (I2MTC), May 2014, pp. 1580-1583.

[47] A. Sánchez, S. Blanc, P. Yuste, A. Perles, and J. J. Serrano, “An ultra-low power and flexible acoustic modem design to develop energy-efficient underwater sensor networks," Sensors, vol. 12, no. 6, pp. 6837-6856, 2012.

48] J. Mathews, M. Barnes, A. Young, and D. K. Arvind, "Low power wake-up in wireless sensor networks using free space optical communications," in Proc. 4th Int. Conf. Sensor Technol. Appl. (SENSORCOMM), Venice, Italy, Jul. 2010, pp. 256-261.

[49] G. Kim et al., "A 695 pW standby power optical wake-up receiver for wireless sensor nodes," in Proc. IEEE Custom Integr. Circuits Conf., San Jose, CA, USA, Sep. 2012, pp. 1-4.

[50] J. Oller, I. Demirkol, J. Casademont, and J. Paradells, "Design, development, and performance evaluation of a low-cost, low-power wake-up radio system for wireless sensor networks," ACM Trans. Sensor Netw., vol. 10, no. 1, pp. 1-24, 2013.

[51] T. V. Prabhakar, N. S. Soumya, P. Muralidharan, and H. S. Jamadagni, "A novel wake-up radio WSN mote," in Proc. Texas Instrum. India Educators Conf. (TIIEC), Apr. 2013, pp. 362-368.

[52] A. S. Boaventura and N. B. Carvalho, "A low-power wakeup radio for application in WSN-based indoor location systems," Int. J. Wireless Inf. Netw., vol. 20, no. 1, pp. 67-73, 2013.

[53] J. Oller et al., "IEEE 802.11-enabled wake-up radio system: Design and performance evaluation," Electron. Lett., vol. 50, no. 20, pp. 1484-1486, Sep. 2014.

[54] D. Spenza et al., "Beyond duty cycling: Wake-up radio with selective awakenings for long-lived wireless sensing systems," in Proc. IEEE Conf. Comput. Commun. (INFOCOM), Apr. 2015, pp. 522-530.

[55] S. Bdiri and F. Derbel, "A nanowatt wake-up receiver for industrial production line," in Proc. 11th Int. Multi Conf. Syst. Signals Devices (SSD), Barcelona, Spain, Feb. 2014, pp. 1-6.
[56] J. Oller et al., "Performance evaluation and comparative analysis $33 з 8$ of subcarrier modulation wake-up radio systems for energy-efficient 3339 wireless sensor networks," Sensors, vol. 14, no. 1, pp. 22-51, 2014. 3340

[57] J. Robert, T. Lindner, and H. Milosiu, "Sub $10 \mu \mathrm{W}$ wake-up-receiver 3341 based indoor/outdoor asset tracking system," in Proc. IEEE 20th Conf. 3342 Emerg. Technol. Factory Autom. (ETFA), Sep. 2015, pp. 1-3. 3343

[58] J. Petäjäjärvi, K. Mikhaylov, R. Vuohtoniemi, H. Karvonen, and 3344 J. Iinatti, "On the human body communications: Wake-up receiver 3345 design and channel characterization," EURASIP J. Wireless Commun. 3346 Netw., vol. 2016, no. 1, p. 179, 2016.

3347

[59] J. M. Rabaey et al., "PicoRadios for wireless sensor networks: The next 3348 challenge in ultra-low power design," in Proc. IEEE Int. Solid-State 3349 Circuits Conf. (ISSCC), vol. 1. San Francisco, CA, USA, Feb. 2002, 3350 pp. 200-201.

[60] N. Pletcher, S. Gambini, and J. Rabaey, "A $65 \mu \mathrm{W}, 1.9$ GHz RF 3352 to digital baseband wakeup receiver for wireless sensor nodes," in 3353 Proc. Custom Integr. Circuits Conf. (CICC), San Jose, CA, USA, 2007, 3354 pp. 539-542.

[61] X. Yu, J.-S. Lee, C. Shu, and S.-G. Lee, "A $53 \mu$ W super-regenerative 3356 receiver for $2.4 \mathrm{GHz}$ wake-up application," in Proc. Asia-Pac. Microw. 3357 Conf., Dec. 2008, pp. 1-4.

[62] N. M. Pletcher, S. Gambini, and J. Rabaey, "A $52 \mu \mathrm{W}$ wake-up receiver 3359 with $-72 \mathrm{dBm}$ sensitivity using an uncertain-IF architecture," IEEE J. 3360 Solid-State Circuits, vol. 44, no. 1, pp. 269-280, Jan. 2009. 3361

[63] T. Takiguchi et al., "A novel wireless wake-up mechanism for energy- $3 з 62$ efficient ubiquitous networks," in Proc. IEEE Int. Conf. Commun. 3363 Workshops, Dresden, Germany, Jun. 2009, pp. 1-5.

[64] R. van Langevelde et al., "An ultra-low-power 868/915 MHz RF 3365 transceiver for wireless sensor network applications," in Proc. IEEE 3366 Radio Frequency Integr. Circuits Symp., Boston, MA, USA, Jun. 2009, 3367 pp. 113-116.

[65] S. Drago et al., "A 2.4GHz 830pJ/bit duty-cycled wake-up receiver 3369 with $-82 \mathrm{dBm}$ sensitivity for crystal-less wireless sensor nodes," in 3370 IEEE Int. Solid-State Circuits Conf. Dig. Tech. Papers (ISSCC), 3371 Feb. 2010, pp. 224-225.

3372

[66] Y. Zhang et al., "A $3.72 \mu \mathrm{W}$ ultra-low power digital baseband for wake- 3373 up radios," in Proc. Int. Symp. VLSI Design Autom. Test (VLSI DAT), 3374 Apr. 2011, pp. 1-4.

[67] C. Hambeck, S. Mahlknecht, and T. Herndl, "A $2.4 \mu \mathrm{W}$ wake-up 3376 receiver for wireless sensor nodes with $-71 \mathrm{dBm}$ sensitivity," in Proc. 3377 IEEE Int. Symp. Circuits Syst. (ISCAS), May 2011, pp. 534-537. 3378

[68] S. Tang, H. Yomo, Y. Kondo, and S. Obana, "Wake-up receiver for 3379 radio-on-demand wireless LANs," EURASIP J. Wireless Commun. 3380 Netw., vol. 2012, no. 1, pp. 1-13, 2012.

[69] X. Li, P. Baltus, D. Milosevic, P. van Zeijl, and A. van Roermund, 3382 "A $60 \mathrm{GHz}$ ultra low-power wake-up radio," in Proc. IEEE Radio 3383 Wireless Symp., Phoenix, AZ, USA, Jan. 2011, pp. 343-346. 3384

[70] D.-Y. Yoon et al., "A new approach to low-power and low-latency 3385 wake-up receiver system for wireless sensor nodes," IEEE J. Solid-State 3386 Circuits, vol. 47, no. 10, pp. 2405-2419, Oct. 2012.

[71] H. Cho, J. Bae, and H.-J. Yoo, “A $37.5 \mu \mathrm{W}$ body channel communica- 3388 tion wake-up receiver with injection-locking ring oscillator for wireless 3389 body area network," IEEE Trans. Circuits Syst. I, Reg. Papers, vol. 60, $з 390$ no. 5, pp. 1200-1208, May 2013.

[72] T. Wada, M. Ikebe, and E. Sano, "60-GHz, $9 \mu \mathrm{W}$ wake-up receiver for 3392 short-range wireless communications," in Proc. ESSCIRC, Bucharest, 3393 Romania, Sep. 2013, pp. 383-386.

[73] P. Jean-François, B. Jean-Jules, and S. Yvon, "Modeling, design and 3395 implementation of a low-power FPGA based asynchronous wake- 3396 up receiver for wireless applications," Analog Integr. Circuits Signal 3397 Process., vol. 77, no. 2, pp. 169-182, 2013.

[74] H. Milosiu et al., "A 3- $\mu \mathrm{W}$ 868-MHz wake-up receiver with $-83 \mathrm{dBm} 3399$ sensitivity and scalable data rate," in Proc. ESSCIRC, Bucharest, 3400 Romania, Sep. 2013, pp. 387-390.

[75] H. Kim and H. Shin, "A 2.4-GHz current-reuse OOK wake-up receiver 3402 for MICS applications," IEICE Electron. Exp., vol. 10, no. 14, 2013, 3403 Art. no. 20130293.

3404

[76] N. Patel, S. Anand, and P. Bhattacharya, "Design of low power wake- 3405 up receiver for wireless sensor network," Int. J. Comput. Appl., vol. 90, 3406 no. 10, pp. 20-25, 2014.

[77] C. Bryant and H. Sjoland, "A $2.45 \mathrm{GHz}, 50 \mu \mathrm{W}$ wake-up receiver front- 3408 end with $-88 \mathrm{dBm}$ sensitivity and 250kbps data rate," in Proc. 40th 3409 Eur. Solid State Circuits Conf. (ESSCIRC), Venice, Italy, Sep. 2014, 3410 pp. 235-238.

3411

[78] T. Abe et al., "An ultra-low-power 2-step wake-up receiver for IEEE 3412 802.15.4G wireless sensor networks," in VLSI Circuits Dig. Tech. 3413 Papers Symp., Honolulu, HI, USA, Jun. 2014, pp. 1-2. 
[79] J. Choi et al., "A 5.8-GHz DSRC transceiver with a $10 \mu \mathrm{A}$ interferenceaware wake-up receiver for the Chinese ETCS," IEEE Trans. Microw. Theory Techn., vol. 62, no. 12, pp. 3146-3160, Dec. 2014.

80] Fraunhofer. (2015). Low Current WakeUp-Receiver for the Internet of Things. [Online]. Available: http://www.iis.fraunhofer.de/ en/ff/icdea/leist/asic/wakeup.html

[81] S. Moazzeni, M. Sawan, and G. E. R. Cowan, "An ultra-low-power energy-efficient dual-mode wake-up receiver," IEEE Trans. Circuits Syst. I, Reg. Papers, vol. 62, no. 2, pp. 517-526, Feb. 2015.

[82] H. Milosiu, F. Oehler, M. Eppel, D. Fruehsorger, and T. Thoenes, "A $7 \mu \mathrm{W} 2.4-\mathrm{GHz}$ wake-up receiver with $-80 \mathrm{dBm}$ sensitivity and high co-channel interferer tolerance," in Proc. IEEE Topical Conf. Wireless Sensors Sensor Netw. (WiSNet), San Diego, CA, USA, Jan. 2015, pp. 35-37.

[83] S.-E. Chen, C.-L. Yang, and K.-W. Cheng, "A 4.5 $\mu \mathrm{W} 2.4 \mathrm{GHz}$ wake-up receiver based on complementary current-reuse RF detector," in Proc. IEEE Int. Symp. Circuits Syst. (ISCAS), Lisbon, Portugal, May 2015, pp. 1214-1217.

[84] T. Taris, H. Kraimia, D. Belot, and Y. Deval, "An FSK and OOK compatible RF demodulator for wake up receivers," J. Low Power Electron. Appl., vol. 5, no. 4, pp. 274-290, 2015.

[85] M.-T. Hoang, N. Sugii, and K. Ishibashi, "A $1.36 \mu \mathrm{W} 312-315 \mathrm{MHz}$ synchronized-OOK receiver for wireless sensor networks using 65 nm SOTB CMOS technology," Solid State Electron., vol. 117, pp. 161-169, Mar. 2016.

[86] A. Nikoofard and S. Mandal, "An $11.5 \mathrm{nW}$ broadband wake-up RF receiver with $-60 \mathrm{dBm}$ sensitivity at $50 \mathrm{MHz}$," in Proc. IEEE Int. Symp. Circuits Syst. (ISCAS), Montreal, QC, Canada, May 2016, pp. 2787-2790.

[87] S. Bdiri and D. Faouzi, "An ultra-low power wake-up receiver for real-time constrained wireless sensor networks," in Proc. AMA Conf., Nuremberg, Germany, May 2015, pp. 612-617.

[88] S. von der Mark and G. Boeck, "Ultra low power wakeup detector for sensor networks," in Proc. SBMO/IEEE MTT-S Int. Microw. Optoelectron. Conf. (IMOC), Oct. 2007, pp. 865-868.

89] C. Tzschoppe, R. Kostack, and F. Ellinger, "A $2.4 \mathrm{GHz}$ fast settling wake-up receiver frontend," in Proc. 10th Conf. Ph.D. Res. Microelectron. Electron. (PRIME), Grenoble, France, Jun. 2014, pp. $1-4$.

[90] R. Jurdak, A. G. Ruzzelli, and G. M. P. O’Hare, "Radio sleep mode optimization in wireless sensor networks," IEEE Trans. Mobile Comput., vol. 9, no. 7, pp. 955-968, Jul. 2010.

[91] AS3933 3D Low Frequency Wakeup Receiver, Austria Micro Syst., Unterpremstätten, Austria, 2015. [Online]. Available: www.ams.com/LF-Receiver/AS3933

[92] Microsemi. (2015). Wireless for Implantable Medical Devices ZL70103 MICS-Band RF Transceiver. [Online]. Available: www.microsemi.com

[93] L. Gu and J. A. Stankovic, "Radio-triggered wake-up for wireless sensor networks," Real Time Syst., vol. 29, nos. 2-3, pp. 157-182, 2005.

[94] C. Shekhar, S. Varma, and M. Radhakrishna, "A passive wake-up circuit for event driven wireless sensor network applications," J. Circuits Syst. Comput., vol. 24, no. 8, 2015, Art. no. 1550120.

95] C. Chung, Y.-H. Kim, T.-H. Ki, K. Bae, and J. Kim, "Fully integrated ultra-low-power $900 \mathrm{MHz}$ RF transceiver for batteryless wireless microsystems," in Proc. 18th IEEE Int. Conf. Electron. Circuits Syst. (ICECS), Beirut, Lebanon, Dec. 2011, pp. 196-199.

[96] N. E. Roberts et al., "A $236 \mathrm{nW}-56.5 \mathrm{dBm}$-sensitivity Bluetooth lowenergy wakeup receiver with energy harvesting in 65nm CMOS," in Proc. IEEE Int. Solid-State Circuits Conf. (ISSCC), San Francisco, CA, USA, Jan. 2016, pp. 450-451.

[97] P. Kamalinejad et al., "A high-sensitivity fully passive wake-up radio front-end for wireless sensor nodes," in Proc. IEEE Int. Conf. Consum. Electron. (ICCE), Las Vegas, NV, USA, 2014, pp. 209-210.

[98] M. Zgaren, A. Moradi, G. Wang, and M. Sawan, "Low-power, highdata rate $915 \mathrm{MHz}$ transceiver with fully passive wake-up receiver for biomedical implants," in Proc. IEEE Int. Conf. Ubiquitous Wireless Broadband (ICUWB), Montreal, QC, Canada, Oct. 2015, pp. 1-4.

[99] E. Nilsson and C. Svensson, "Ultra low power wake-up radio using envelope detector and transmission line voltage transformer," IEEE J. Emerg. Sel. Topics Circuits Syst., vol. 3, no. 1, pp. 5-12, Mar. 2013.

100] L. Chen, J. Warner, W. Heinzelman, and I. Demirkol, "MH-REACHMote: Supporting multi-hop passive radio wake-up for wireless sensor networks," in Proc. IEEE Int. Conf. Commun. (ICC), London, U.K., Jun. 2015, pp. 6512-6518.

101] D. D. Donno, L. Catarinucci, and L. Tarricone, "Ultralong-range RFIDbased wake-up radios for wireless sensor networks," IEEE Sensors J., vol. 14, no. 11, pp. 4016-4017, Nov. 2014.
[102] H. Ba, I. Demirkol, and W. Heinzelman, "Passive wake-up radios: From 3493 devices to applications," Ad Hoc Netw., vol. 11, no. 8, pp. 2605-2621, 3494 2013.

[103] Y. Ammar, S. Bdiri, and F. Derbel, "An ultra-low power wake up 3496 receiver with flip flops based address decoder," in Proc. 12th Int. Multi 3497 Conf. Syst. Signals Devices (SSD), Mahdia, Tunisia, 2015, pp. 1-5. 3498

[104] S. J. Marinkovic and E. M. Popovici, "Nano-power wireless wake-up 3499 receiver with serial peripheral interface," IEEE J. Sel. Areas Commun., 3500 vol. 29, no. 8, pp. 1641-1647, Sep. 2011.

[105] M. D. Prete, D. Masotti, A. Costanzo, M. Magno, and L. Benini, 3502 "A $2.4 \mathrm{GHz}-868 \mathrm{MHz}$ dual-band wake-up radio for wireless sensor 3503 network and IoT," in Proc. IEEE 11th Int. Conf. Wireless Mobile 3504 Comput. Netw. Commun. (WiMob), Abu Dhabi, UAE, Oct. 2015, 3505 pp. $322-328$.

3506

[106] N. Sumathi and B. Venkatalakshmi, "Design of $433 \mathrm{MHz}$ compati- 3507 ble matching network of wake-up receiver for wireless sensor node," 3508 in Proc. Glob. Conf. Commun. Technol. (GCCT), Thuckalay, India, 3509 Apr. 2015, pp. 807-811.

[107] T. Polonelli, T. L. Huy, L. Lizzi, F. Ferrero, and M. Magnod, "A wake- 3511 up receiver with ad-hoc antenna co-design for wearable applications," 3512 in Proc. IEEE Sensors Appl. Symp. (SAS), Catania, Italy, Apr. 2016, 3513 pp. 1-6.

[108] Y. Wang, H. Wang, and G. Wen, "A low-power edge detection tech- 3515 nique for sensor wake-up applications," J. Circuits Syst. Comput., 3516 vol. 24, no. 10, 2015, Art. no. 1550157.

3517

[109] H. Sjoland et al., "A receiver architecture for devices in wireless body 3518 area networks," IEEE J. Emerg. Sel. Topics Circuits Syst., vol. 2, no. 1, 3519 pp. 82-95, Mar. 2012.

3520

[110] P. N. Thanh, K. N. Tuan, and X. M. Dong, "A 100- $\mu$ W wake- 3521 up receiver for UHF transceiver," in Proc. Int. Conf. IC Design 3522 Technol. (ICICDT), Jun. 2016, pp. 1-4.

[111] N. E. Roberts and D. D. Wentzloff, "A $98 \mathrm{nW}$ wake-up radio for 3524 wireless body area networks," in Proc. IEEE Radio Frequency Integr. 3525 Circuits Symp. (RFIC), Montreal, QC, Canada, Jun. 2012, pp. 373-376. 3526

[112] J. Lim, H. Cho, K. Cho, and T. Park, "High sensitive RF-DC rectifier 3527 and ultra low power DC sensing circuit for waking up wireless system," 3528 in Proc. Asia-Pac. Microw. Conf., Singapore, Dec. 2009, pp. 237-240. 3529

[113] C. Salazar, A. Kaiser, A. Cathelin, and J. Rabaey, "13.5 A -97dBm- 3530 sensitivity interferer-resilient $2.4 \mathrm{GHz}$ wake-up receiver using dual- 3531 IF multi-N-Path architecture in 65nm CMOS," in Proc. IEEE 3532 Int. Solid-State Circuits Conf. (ISSCC), San Francisco, CA, USA, 3533 Feb. 2015, pp. 1-3.

[114] J.-Y. Hsieh, Y.-C. Huang, P.-H. Kuo, T. Wang, and S.-S. Lu, "A 0.45-V 3535 low-power OOK/FSK RF receiver in $0.18 \mu \mathrm{m}$ CMOS technology for 3536 implantable medical applications," IEEE Trans. Circuits Syst. I, Reg. 3537 Papers, vol. 63, no. 8, pp. 1123-1130, Aug. 2016.

[115] X. Huang, S. Rampu, X. Wang, G. Dolmans, and H. de Groot, 3539 "A $2.4 \mathrm{GHz} / 915 \mathrm{MHz} 51 \mu \mathrm{W}$ wake-up receiver with offset and noise 3540 suppression," in IEEE Int. Solid-State Circuits Conf. Dig. Tech. 3541 Papers (ISSCC), San Francisco, CA, USA, Feb. 2010, pp. 222-223. 3542

[116] S. Oh, N. E. Roberts, and D. D. Wentzloff, "A 116nW multi-band 3543 wake-up receiver with 31-bit correlator and interference rejection," in 3544 Proc. IEEE Custom Integr. Circuits Conf. (CICC), San Jose, CA, USA, 3545 Sep. 2013, pp. 1-4.

[117] M. S. Durante and S. Mahlknecht, "An ultra low power wakeup receiver 3547 for wireless sensor nodes," in Proc. 3rd Int. Conf. Sensor Technol. 3548 Appl. (SENSORCOMM), Athens, Greece, 2009, pp. 167-170. 3549

[118] Y. Shuangming, F. Peng, and W. Nanjian, "A low power non-volatile 3550 LR-WPAN baseband processor with wake-up identification receiver," 3551 China Commun., vol. 13, no. 1, pp. 33-46, Jan. 2016.

[119] M. Malinowski, M. Moskwa, M. Feldmeier, M. Laibowitz, and 3553 J. A. Paradiso, "CargoNet: A low-cost micropower sensor node exploit- 3554 ing quasi-passive wakeup for adaptive asychronous monitoring of 3555 exceptional events," in Proc. 5th Int. Conf. Embedded Netw. Sensor 3556 Syst. (SenSys), Sydney, NSW, Australia, 2007, pp. 145-159. 3557

[120] T. Hakkinen and J. Vanhala, "Ultra-low power wake-up circuit for 3558 short-range wireless communication," in Proc. IET 4th Int. Conf. Intell. 3559 Environ., Seattle, WA, USA, Jul. 2008, pp. 1-4.

[121] A. Bannoura et al., "Acoustic wake-up receivers for home automation 3561 control applications," Electronics, vol. 5, no. 1, p. 4, 2016.

[122] C. Carrascal, I. Demirkol, and J. Paradells, "On-demand sensor node 3563 wake-up using solar panels and visible light communication," Sensors, 3564 vol. 16, no. 3, p. 418, 2016.

[123] W. Lim et al., "A $380 p W$ dual mode optical wake-up receiver 3566 with ambient noise cancellation," in Proc. IEEE Symp. VLSI 3567 Circuits (VLSI-Circuits), Honolulu, HI, USA, Jun. 2016, pp. 1-2. 3568 
3569 [124] D. Lee and P. P. Mercier, "Introduction to ultra low power transceiver 3570 design,” in Ultra-Low-Power Short-Range Radios. Cham, Switzerland: $3571 \quad$ Springer, 2015, pp. 1-23.

3572 [125] C. Guo, L. C. Zhong, and J. M. Rabaey, "Low power dis3573 tributed MAC for ad hoc sensor radio networks," in Proc. Glob. 3574 Telecommun. Conf. (GLOBECOM), vol. 5. San Antonio, TX, USA, 3575 2001, pp. 2944-2948.

3576 [126] C. Schurgers, V. Tsiatsis, S. Ganeriwal, and M. Srivastava, "Optimizing 3577 sensor networks in the energy-latency-density design space," IEEE 3578 Trans. Mobile Comput., vol. 1, no. 1, pp. 70-80, Jan./Mar. 2002.

3579 [127] X. Yang and N. H. Vaidya, "A wakeup scheme for sensor networks: 3580 Achieving balance between energy saving and end-to-end delay," in $3581 \quad$ Proc. 10th IEEE Real-Time Embedded Technol. Appl. Symp. (RTAS), 3582 Toronto, ON, Canada, 2004, pp. 19-26.

3583 [128] M. J. Miller and N. H. Vaidya, "A MAC protocol to reduce sen3584 sor network energy consumption using a wakeup radio," IEEE Trans. 3585 Mobile Comput., vol. 4, no. 3, pp. 228-242, May/Jun. 2005.

586 [129] I. Khalil, S. Bagchi, and N. B. Shroff, "SLAM: Sleep-wake aware 3587 local monitoring in sensor networks," in Proc. 37th Annu. IEEE/IFIP Int. Conf. Depend. Syst. Netw. (DSN), Edinburgh, U.K., Jun. 2007, pp. $565-574$

[130] S. Mahlknecht and M. S. Durante, "WUR-MAC: Energy efficient wakeup receiver based MAC protocol," in Proc. 8th Conf. Fieldbuses Netw. Ind. Embedded Syst. (IFAC), vol. 42. 2009, pp. 79-83.

[131] N. S. Mazloum and O. Edfors, "DCW-MAC: An energy efficient medium access scheme using duty-cycled low-power wakeup receivers," in Proc. IEEE Veh. Technol. Conf. (VTC Fall), San Francisco, CA, USA, Sep. 2011, pp. 1-5.

132] N. S. Mazloum and O. Edfors, "Performance analysis and energy optimization of wake-up receiver schemes for wireless low-power applications," IEEE Trans. Wireless Commun., vol. 13, no. 12, pp. 7050-7061, Dec. 2014.

133] N. Ullah, P. Khan, and K. S. Kwak, "A very low power MAC (VLPM) protocol for wireless body area networks," Sensors, vol. 11, no. 4 pp. 3717-3737, 2011.

[134] M. A. Ameen, N. Ullah, M. S. Chowdhury, and K. Kwak, "A MAC protocol for body area networks using out-of-band radio," in Proc. 11th Eur. Wireless Conf. Sustain. Wireless Technol. (Eur. Wireless), Vienna, Austria, Apr. 2011, pp. 1-6.

[135] M. A. Ameen, N. Ullah, and K. Kwak, "Design and analysis of a MAC protocol for wireless body area network using wakeup radio," in Proc. 11th Int. Symp. Commun. Inf. Technol. (ISCIT), Hangzhou, China, Oct. 2011, pp. 148-153.

[136] J. Blanckenstein, J. Klaue, and H. Karl, "Energy efficient clustering using a wake-up receiver," in Proc. 18th Eur. Wireless Conf., Poznań, Poland, Apr. 2012, pp. 1-8.

[137] T. N. Le et al., "Ultra low power asynchronous MAC protocol using wake-up radio for energy neutral WSN," in Proc. 1st Int. Workshop Energy Neutral Sens. Syst. (ENSSys), Rome, Italy, 2013, pp. 1-6.

[138] H. Karvonen, J. Petäjäjärvi, J. Iinatti, M. Hämäläinen, and C. Pomalaza-Ráez, "A generic wake-up radio based MAC protocol for energy efficient short range communication," in Proc. IEEE 25th Annu. Int. Symp. Pers. Indoor Mobile Radio Commun. (PIMRC), Washington, DC, USA, Sep. 2014, pp. 2173-2177.

139] H. Karvonen, J. Iinatti, J. Petäjäjärvi, and M. Hämäläinen, "Energy efficient IR-UWB WBAN using a generic wake-up radio based MAC protocol," in Proc. 9th Int. Conf. Body Area Netw. (BodyNets), London, U.K., 2014, pp. 358-364.

[140] J. Lebreton and N. Murad, "Implementation of a wake-up radio cross-layer protocol in OMNeT++/MiXiM," in Proc. 2nd OMNeT++ Community Summit, Sep. 2015, pp. 1-5.

[141] T. N. Le, A. Pegatoquet, and M. Magno, "Asynchronous on demand MAC protocol using wake-up radio in wireless body area network," in Proc. 6th IEEE Int. Workshop Adv. Sensors Interfaces (IWASI), Jun. 2015, pp. 228-233.

[142] F. Dressler et al., "Monitoring bats in the wild: On using erasure codes for energy-efficient wireless sensor networks," ACM Trans. Sensor Netw. (TOSN), vol. 12, no. 1, pp. 1-29, Mar. 2016.

43] R. Piyare, T. Istomin, and A. L. Murphy, "WaCo: A wake-up radio COOJA extension for simulating ultra low power radios," in Proc. 14th Int. Conf. Embedded Wireless Syst. Netw. (EWSN), Uppsala, Sweden, Feb. 2017, pp. 48-53.

[144] R. Weigel. BATS: Dynamic Adaptable Applications for Bats Tracking by Embedded Communicating Systems. [Online]. Available: http://www.for-bats.org/
[145] F. Dressler et al., "Protocol design for ultra-low power wake-up systems 3644 for tracking bats in the wild," in Proc. IEEE Int. Conf. Commun. (ICC), 3645 London, U.K., Jun. 2015, pp. 6345-6350.

[146] F. Dressler et al., "From radio telemetry to ultra-low-power sensor 3647 networks: Tracking bats in the wild," IEEE Commun. Mag., vol. 54, 3648 no. 1, pp. 129-135, Jan. 2016.

[147] M. Magno et al., "Smart power unit with ultra low power radio trigger 3650 capabilities for wireless sensor networks," in Proc. Conf. Design Autom. 3651 Test Europe (DATE), Dresden, Germany, 2012, pp. 75-80. 3652

[148] T. Stathopoulos et al., "End-to-end routing for dual-radio 3653 sensor networks," in Proc. 26th IEEE Int. Conf. Comput. 3654 Commun. (INFOCOM), May 2007, pp. 2252-2260. 3655

[149] M. Vodel, M. Lippmann, and W. Hardt, "WRTA-Wake-up-receiver 3656 optimised routing and topology optimisation approach," in Proc. 3657 Int. Conf. ITS Telecommun. (ITST), Taipei, Taiwan, Nov. 2012, 3658 pp. 329-334.

[150] O. Gnawali et al., "CTP: An efficient, robust, and reliable collec- 3660 tion tree protocol for wireless sensor networks," ACM Trans. Sensor 3661 Netw. (TOSN), vol. 10, no. 1, pp. 1-49, Dec. 2013.

[151] S. Basagni, C. Petrioli, and D. Spenza, "CTP-WUR: The collection tree 3663 protocol in wake-up radio WSNs for critical applications," in Proc. Int. 3664 Conf. Comput. Netw. Commun. (ICNC), Kauai, HI, USA, Feb. 2016, 3665 pp. $1-6$.

[152] R. C. Shah and J. M. Rabaey, "Energy aware routing for low energy 3667 ad hoc sensor networks," in Proc. IEEE Wireless Commun. Netw. 3668 Conf. (WCNC), vol. 1. Orlando, FL, USA, Mar. 2002, pp. 350-355. 3669

[153] L. Song and D. Hatzinakos, "A cross-layer architecture of wireless 3670 sensor networks for target tracking," IEEE/ACM Trans. Netw., vol. 15, 3671 no. 1, pp. 145-158, Feb. 2007.

3672

[154] L. Catarinucci et al., "A cross-layer approach to minimize the energy 3673 consumption in wireless sensor networks," Int. J. Distrib. Sensor Netw., 3674 vol. 2014, Jan. 2014.

[155] F. A. Aoudia, M. Gautier, and O. Berder, "OPWUM: Opportunistic 3676 MAC protocol leveraging wake-up receivers in WSNs," J. Sensors, 3677 vol. 501, pp. 1-9, 2016.

[156] T. Kumberg, M. Schink, L. M. Reindl, and C. Schindelhauer, 3679 "T-ROME: A simple and energy efficient tree routing protocol for 3680 low-power wake-up receivers," Ad Hoc Netw., vol. 59, pp. 97-115, 3681 May 2017.

[157] C. Petrioli, M. Nati, P. Casari, M. Zorzi, and S. Basagni, “ALBA-R: 3683 Load-balancing geographic routing around connectivity holes in wire- 3684 less sensor networks," IEEE Trans. Parallel Distrib. Syst., vol. 25, no. 3, 3685 pp. 529-539, Mar. 2014.

[158] D. Alessandrelli et al., "Performance evaluation of an energy-efficient 3687 MAC scheduler by using a test bed approach," J. Commun. Softw. Syst., 3688 vol. 9, no. 1, pp. 84-96, 2013.

3689

[159] A. W. Min and M. Park, "Connectivity management for mobile wireless 3690 low-power wake-up radios," U.S. Patent 14/803 914, Jan. 26, 2017.3691

[160] A. T. Capossele, V. Cervo, C. Petrioli, and D. Spenza, "Counteracting 3692 denial-of-sleep attacks in wake-up-radio-based sensing systems," in 3693 Proc. 13th Annu. IEEE Int. Conf. Sens. Commun. Netw. (SECON), 3694 London, U.K., Jun. 2016, pp. 1-9.

[161] R. Cavallari, F. Martelli, R. Rosini, C. Buratti, and R. Verdone, "A sur- 3696 vey on wireless body area networks: Technologies and design chal- 3697 lenges," IEEE Commun. Surveys Tuts., vol. 16, no. 3, pp. 1635-1657, 3698 3rd Quart., 2014.

[162] P. Sthapit and J.-Y. Pyun, "Effects of radio triggered sensor MAC pro- 3700 tocol over wireless sensor network," in Proc. IEEE 11th Int. Conf. 3701 Comput. Inf. Technol. (CIT), Paphos, Cyprus, Aug. 2011, pp. 546-551. 3702

[163] M. Patel and J. Wang, "Applications, challenges, and prospective in 3703 emerging body area networking technologies," IEEE Wireless Commun. 3704 Mag., vol. 17, no. 1, pp. 80-88, Feb. 2010.

3705

[164] S. Van Roy, "Dynamic channel modeling for multi-sensor body 3706 area networks," IEEE Trans. Antennas Propag., vol. 61, no. 4, 3707 pp. 2200-2208, Apr. 2013.

[165] M. Dohler, T. Watteyne, T. Winter, and D. Barthel, "Routing require- 3709 ments for urban low-power and lossy networks," Internet Eng. Task 3710 Force, Fremont, CA, USA, RFC 5548, May 2009.

[166] J. Paradells, C. Gomez, I. Demirkol, J. Oller, and M. Catalan, 3712 "Infrastructureless smart cities. Use cases and performance," in 3713 Proc. Int. Conf. Smart Commun. Netw. Technol. (SaCoNeT), 3714 Vilanova i la Geltrú, Spain, Jun. 2014, pp. 1-6.

[167] T. Kumberg, J. Kokert, V. Younesi, S. Koenig, and L. M. Reindl, 3716 "Wake-up transceivers for structural health monitoring of bridges," in 3717 Proc. SPIE Smart Struct. Mater. Nondestruct. Eval. Health Monitor, 3718 vol. 9804. Las Vegas, NV, USA, 2016, pp. 1-9. 
3720 [168] G. U. Gamm and L. M. Reindl, "Smart metering using distributed 3721 wake-up receivers," in Proc. IEEE Int. Instrum. Meas. Technol. 3722 Conf. (I2MTC), Graz, Austria, May 2012, pp. 2589-2593.

3723 [169] K.-H. Chang and B. Mason, "The IEEE 802.15.4g standard for smart metering utility networks," in Proc. IEEE 3rd Int. Conf. Smart Grid Commun. (SmartGridComm), Tainan, Taiwan, Nov. 2012, pp. 476-480.

170] T. Khalifa, K. Naik, and A. Nayak, "A survey of communication protocols for automatic meter reading applications," IEEE Commun. Surveys Tuts., vol. 13, no. 2, pp. 168-182, 2nd Quart., 2011.

[171] G. P. Picco et al., "Geo-referenced proximity detection of wildlife with WildScope: Design and characterization," in Proc. 14th Int. Conf. Inf. Process. Sensor Netw. (IPSN), Seattle, WA, USA, 2015, pp. 238-249.

[172] M. Magno, F. Tombari, D. Brunelli, L. D. Stefano, and L. Benini, "Multimodal video analysis on self-powered resource-limited wireless smart camera," IEEE J. Emerg. Sel. Topic Circuits Syst., vol. 3, no. 2, pp. 223-235, Jun. 2013

173] N. Simon et al., "Indoor localization system for emergency responders with ultra low-power radio landmarks," in Proc. IEEE Int. Instrum. Meas. Technol. Conf. (I2MTC), Pisa, Italy, May 2015, pp. 309-314.

174] H. Liu, M. Bolic, A. Nayak, and I. Stojmenovi, "Integration of RFID and wireless sensor networks," in Encyclopedia on Ad Hoc and Ubiquitous Computing: Theory and Design of Wireless Ad Hoc Sensor, and Mesh Networks. Singapore: World Sci., 2010, pp. 319-348.

175] F. Casamassima, M. Magno, E. Farella, and L. Benini, "Context aware power management enhanced by radio wake up in body area networks," in Proc. IEEE 13th Int. Conf. Embedded Ubiquitous Comput., Porto, Portugal, Oct. 2015, pp. 76-82.

[176] M. Kuba, "Wake-up transceiver protocol for power line communications," in Proc. IEEE Int. Symp. Power Line Commun. Appl. (ISPLC) Austin, TX, USA, Mar./Apr. 2015, pp. 125-130.

177] P. Golchin, P. Tosato, and D. Brunelli, "Design optimization of zero power wake-up receiver in power line communication," in Proc. Int. Symp. Power Electron. Elect. Drives Autom. Motion (SPEEDAM), Jun. 2016, pp. 564-569.

178] D. Brunelli, P. Tosato, and R. Fiorelli, "Design and implementation of zero power wake-up for PLC modems in smart street lighting systems," in Proc. IEEE 20th Conf. Emerg. Technol. Factory Autom. (ETFA), Luxembourg City, Luxembourg, Sep. 2015, pp. 1-7.

179] M. Magno, F. A. Aoudia, M. Gautier, O. Berder, and L. Benini, "WULoRa: An energy efficient IoT end-node for energy harvesting and heterogeneous communication," in Proc. Conf. Design Autom. Test Europe (DATE), Lausanne, Switzerland, Mar. 2017, pp. 1528-1533. Days Indian Ocean (RADIO), Oct. 2016, pp. 1-2.

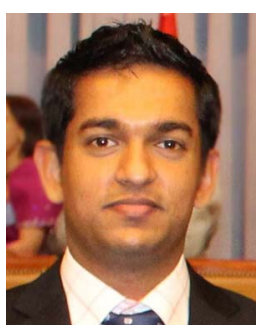

Rajeev Piyare received the M.Eng. degree in 3796 electronics engineering from Mokpo National 3797 University, South Korea, in 2014. He is currently 3798 pursuing the $\mathrm{Ph} . \mathrm{D}$. degree with the University 3799 of Trento and Bruno Kessler Foundation, Italy, 3800 researching on protocols for autonomous low-power 3801 wireless sensor and actuator networks. He was a 3802 Lecturer in electronics with Fiji National University 3803 from 2009 to 2012

3804

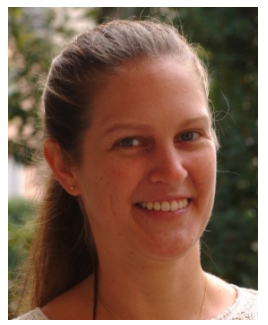

Amy L. Murphy (SM'16) received the B.S. 3805 degree in computer science from the University of 3806 Tulsa in 1995, and the M.S. and D.Sc. degrees 3807 from Washington University, St. Louis, MO, USA, 3808 in 1997 and 2000, respectively. She was an 3809 Assistant Professor with the University of Lugano, 3810 Switzerland, and the University of Rochester, NY, 3811 USA. She is a Research Scientist with the Energy 3812 Efficient Embedded Digital Architectures Unit, 3813 Bruno Kessler Foundation-ICT, a research center 3814 in Trento, Italy. Her research interests include the 3815 design, specification, and implementation of protocols and middleware 3816 systems for wireless environments.

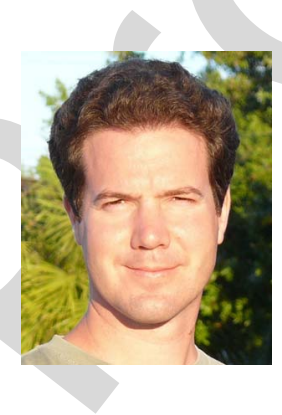

Csaba Kiraly received the degree in informatics and 3818 telecommunications in 2001 and the Ph.D. degree 3819 from the Budapest University of Technology and 3820 Economics. He is a Senior Researcher with Bruno 3821 Kessler Foundation, Trento, Italy. His main research 3822 interests are in design and performance evaluation 3823 of networking protocols, with particular focus on 3824 WSN and IoT systems. He has co-authored over 303825 scientific papers. He has been involved in several 3826 EU projects since FP4, currently being Technical 3827 Coordinator of the H2020 AGILE-IoT Project. 3828

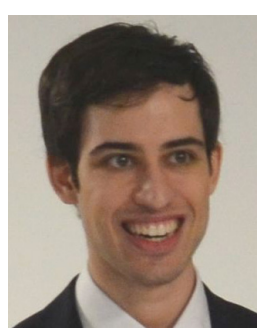

Pietro Tosato (S'15) received the master's degree 3829 from the University of Trento in 2015, where he is 3830 currently pursuing the Ph.D. degree under the super- 3831 vision of Prof. D. Brunelli. His research interests 3832 include smart grid and applications of low-power 3833 technologies for electronics and Internet of Things. 3834

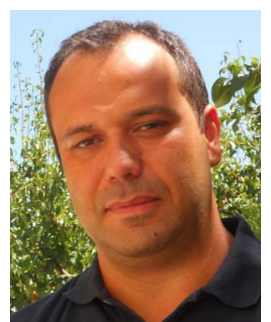

Davide Brunelli (M'10-SM'16) received the M.S. 3835 (cum laude) and $\mathrm{Ph} . \mathrm{D}$. degrees in electrical engineer- 3836 ing from the University of Bologna, Bologna, Italy, 3837 in 2002 and 2007, respectively. He was a Visiting 3838 Researcher with ETH, Zürich, Switzerland, where 3839 he researched on methodologies for energy harvest- 3840 ing aware embedded design. He was a Scientific 3841 Supervisor of several EU FP7 and H2020 projects, 3842 and was leading industrial cooperation activities with 3843 Telecom Italia, Italy, and STMicroelectronics. He is 3844 currently a Tenure Track Associate Professor with 3845 the University of Trento, Trento, Italy. His current research interests include 3846 smart grids and the development of new techniques of energy scavenging for 3847 Internet of Things (IoT) and embedded systems, the optimization of low-power 3848 and low-cost consumer electronics, and the interaction and design issues in 3849 embedded personal and wearable devices. He is leading the activity on energy 3850 efficiency in the IEEE Smart City initiative for the city of Trento. He is a 3851 member of several Technical Program Committees of conferences in the field 3852 of IoT and energy management. 